See discussions, stats, and author profiles for this publication at: https://www.researchgate.net/publication/337335786

\title{
Mapping a cognitive theory onto neurology
}

Research · November 2019

DOI: 10.13140/RG.2.2.28982.24641

CITATION

READS

1

520

1 author:

Lorin Friesen

www.mentalsymmetry.com

5 PUBLICATIONS 2 CITATIONS

SEE PROFILE

Some of the authors of this publication are also working on these related projects:

A Meta-theory of Cognition View project 


\title{
Mapping a cognitive theory onto neurology
}

\author{
Lorin Friesen, mentalsymmetry.com, Abbotsford, BC, Canada
}

doi: 10.13140/RG.2.2.28982.24641

\section{Abstract}

Neurological research has made amazing strides in recent years. Enough is now known about what specific brain areas do to make it possible to start examining how various parts of the brain interact. What is missing is a general theory of cognition to tie all of this information together. Back in the 1980s, a cognitive theory was developed that began with a system of cognitive styles and was expanded through an in-depth study of biographies. It was discovered at that time that this theory mapped in a general way onto the brain. This cognitive theory, known as the theory of mental symmetry, has recently been tested as a meta-theory by using it to analyze a number of fields and theories dealing with human thought and behavior. This paper shows that personality traits that were discovered by mental symmetry correspond in detail to the functioning of brain regions described in current neurological papers. In brief, the cognitive model suggests that there are seven cognitive styles: There are four simple styles, and there are three composite styles that combine the thinking of the simple styles. Two of the simple styles use emotions and emphasize a circuit composed of orbitofrontal cortex, inferior frontal cortex, temporal lobe, and amygdala, with one in the left hemisphere and the other in the right hemisphere. The other two simple styles use confidence and emphasize a circuit consisting of dorsolateral frontal cortex, frontopolar cortex, parietal cortex, and hippocampus, again with one in the left hemisphere and the other in the right hemisphere. The three composite styles form a processing chain. The first composite style combines the two simple emotional styles and emphasizes the ventral striatum, and dopamine. This leads to the second composite style, which combines the two simple confidence styles and emphasizes the anterior cingulate, the dorsal striatum, and serotonin. This is followed by the third composite style which balances the functioning of the mind and emphasizes the thalamus and noradrenaline.

\section{Introduction}

One of the general findings of neurology is that the brain evaluates information using a combination of bottom-up and top-down processing. Specific information from the senses is analyzed in a bottom-up fashion, but this analysis is guided by some internal structure that is being imposed in a top-down fashion. For instance, this happens with visual attention (Melloni, 2012), memory encoding (Uncapher, 2011), emotion generation (Ochsner, 2009), and language (Simanova, 2015). In a similar manner, Thomas Kuhn famously said that all scientific research evaluates specific facts in the light of some paradigm. This principle can be seen in the way that neurologists evaluate neurological data. For instance, the activity of the medial prefrontal cortex has been evaluated from the perspective of social interaction (Wagner, 2012), economic activity (Grabenhorst, 2011), Theory of Mind (Hartwright, 2014), self domains (Araujo, 2015), and moral cognition (Bzdok, 2012). All of these approaches have discovered important insights. But each has discovered different insights, because each is taking facts 
that were discovered using bottom-up analysis and placing these facts in a top-down manner within the mental framework of some paradigm.

This paper will be placing neurological findings within the paradigm of a general theory of cognition known as mental symmetry. The theory of mental symmetry began as a system of cognitive styles that is still taught in some circles (Fortune, 2009), and most of the initial data was collected by an analysis of 200 biographies (Friesen, 1986). Thus, the theory comes from a non-scientific source, it was developed in a semi-rigorous manner, and it has not passed peer review. Despite this uncertain parentage, the theory of mental symmetry corresponds in detail with the latest findings of neurology, and mental symmetry described most of these cognitive traits before they were uncovered by neurology. In addition, mental symmetry has been used as a meta-theory to explain a diverse range of topics, including culture, linguistics (Friesen, 2013), and religion (Friesen, 2015).

This paper will not attempt to prove the theory of mental symmetry. As Thomas Kuhn said, it is not possible to prove a new paradigm, because one is always viewing a potential paradigm through the lens of some other paradigm. "When paradigms enter, as they must, into a debate about paradigm choice, their role is necessarily circular. Each group uses its own paradigm to argue in that paradigm's defense” (Kuhn, 1970, p. 94). Going further, peer-review works well for adding knowledge incrementally to an existing paradigm, but it breaks down when attempting to introduce a new paradigm. In the words of Kuhn, "In so far as he is engaged in normal science, the research worker is a solver of puzzles, not a tester of paradigms.” (Kuhn, 1970, p. 144). Therefore, the theory of mental symmetry will be briefly described and then the functions of the different parts of the brain will be examined in the light of mental symmetry, focusing upon areas of higher cognition. And because this paper will be looking at many different neurological contexts, I will be quoting extensively from the original sources.

\section{Mental Symmetry}

The theory of mental symmetry is summarized by a simple diagram known as the diagram of mental symmetry. The seven cognitive modules are each given a label that describes a major cognitive trait: Teacher, Exhorter, Mercy, Server, Contributor, Perceiver, and Facilitator. As we shall see when going through the neurology, studying personality has revealed a number of details that are not explicitly shown by the diagram of mental symmetry, but this diagram still remains the best way to summarize the traits in a simple manner, and the diagram has been arranged in a manner that maps in a general way onto neurology.

In a similar manner, neurological research has now shifted away from looking merely at parts of the brain in isolation to examining how various parts of the brain interact to form large-scale functioning structures. "A new paradigm is emerging in cognitive neuroscience that moves beyond the simplistic mapping of cognitive constructs onto individual brain areas and emphasizes instead the conjoint function of brain areas working together as large-scale networks” (Bressler, 2010, p. 277). Thus, the seven labels (Teacher, Exhorter, Mercy, etc.) in the diagram of mental symmetry should be viewed as large-scale cognitive modules each composed of several interacting parts. 


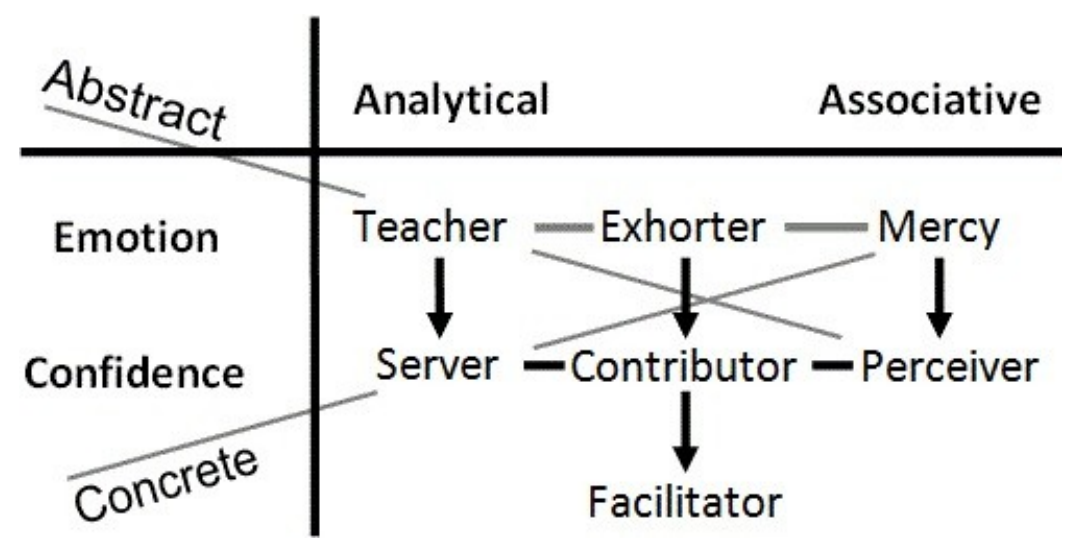

The diagram uses two primary axes: analytic versus associative, and emotion versus confidence. Analytic versus associative corresponds to left hemisphere versus right hemisphere. This is a crude distinction, but it remains a useful one. Many cognitive tasks involve extensive interaction between the corresponding regions in the left and right hemispheres, but the underlying principle remains that the two hemispheres are using different strategies to perform similar tasks. And it appears that these different strategies can be summarized by the terms analytical and associative. Analytical processing focuses upon sequences, time, order, and structure, while associative processing emphasizes objects, space, position, and specifics. On the one hand, these two mental strategies are quite different. But on the other hand, most tasks can be handled using either-or both—of these two strategies.

Emotion versus confidence corresponds best to limbic system versus executive function. These are also crude distinctions which can be refined. For instance, the hippocampus has traditionally been regarded as part of the limbic system, but it deals with memory and not emotion. "The concept of a single limbic system may no longer appropriately reflect how the brain operates. Instead, separate systems, which might be designated the emotional limbic system and the episodic memory hippocampal system, are present in the brain” (Rolls, 2015, p. 148). Confidence and emotion are both labels that the mind applies to information. Confidence describes the certainty with which one knows some fact or skill. And emotion is - well, emotion. As one recent paper complained, "Although extensively studied, an unequivocal definition of emotions is still lacking and the subject of contentions. For example, in the 1980’s, Fehr and Russell wrote that 'Everyone knows what an emotion is, until asked to give a definition. Then, it seems, no one knows.' Kleinginna and Kleinginna considered 92 definitions and 9 skeptical descriptions produced by scientists in the field, which effectively represent the lack of consensus with regard to the characteristics that define the concept of emotion and its usefulness in the scientific framework" (Celeghin, 2017, p. 2). Summarizing, the precise definition of emotion may be uncertain, but it is well-established that there is a limbic system that functions emotionally (if one excludes the hippocampus).

Confidence interacts with emotion, as illustrated by the concept of executive function. One paper, for instance, explains that "Executive function, defined as the ability to hold in mind information in working memory, to inhibit fast and unthinking responses to stimulation, and to flexibly shift the focus of one's mental frame, is more or less the foundation for the intentional, volitional self-directed control of behavior. The cognitive skills that make up this construct help us to limit impulsive responses, to regulate emotions, and to avoid bad decisions that might bring short-term gain but longer-term problems” (Blair, 2016, p. 1). In other words, executive function is needed to shift from one mental 
context to another, but it also describes the ability to use knowledge to guide emotions, and it can be overwhelmed by impulsive emotional responses. This summarizes the interaction between confidence and emotion. Emotion can overwhelm confidence, but one can also gain sufficient confidence to guide emotions.

The diagram of mental symmetry uses the two axes of analytical versus associative and emotion versus confidence to place the four 'simple styles' of Teacher, Mercy, Server, and Perceiver. This means that Teacher is analytical emotional, Mercy is associative emotional, Server is analytical confidence, and Perceiver is associative confidence. If one compares the traits gathered from observing behavior with the functions of brain regions, then one comes up with the following mapping: Teacher thought uses a circuit composed of left orbitofrontal, left inferior frontal, left amygdala, and left temporal, while Mercy thought uses the same regions in the right hemisphere. Server thought uses a circuit including left dorsolateral, left frontopolar, left parietal, and left hippocampus, while Perceiver thought uses the same regions in the right hemisphere.

The two diagonals are labeled abstract and concrete. Teacher and Perceiver thought cooperate to generate abstract thought. Perceiver thought comes up with facts and Teacher thought assembles these facts into general theories. Similarly, Mercy and Server thought cooperate to generate concrete thought. Mercy thought focuses upon experiences and how they feel, while Server thought carries out the actions that are required to reach desirable experiences and avoid unpleasant experiences.

The ‘composite styles' of Exhorter, Contributor, and Facilitator integrate corresponding regions in the two hemispheres. Exhorter combines Teacher and Mercy, and Contributor combines Server and Teacher. Finally, Facilitator 'facilitates' the functioning of the entire mind, emphasizing the relationship between Perceiver and Mercy thought, as well as the relationship between Server and Teacher thought. Exhorter thought uses the ventral striatum and is related to dopamine, Contributor thought uses the cingulate cortex and the dorsal striatum and is related to serotonin. Finally, Facilitator thought uses the thalamus and is probably connected with noradrenaline. Looking at this in terms of a chain of processing, Exhorter thought provides motivation, Contributor thought chooses between various options, while Facilitator thought mixes and adjusts in order to maintain equilibrium. The brain regions that are directly connected with the composite styles are rather small compared to those of the simple styles. However, the composite styles also have partial access to the regions used by the simple styles, but they manipulate this information using a different strategy with the help of the three primary neuromodulators.

Looking at this more specifically, Perceiver thought looks for connections between facts, and Server thought focuses upon relating sequences, whereas Contributor thought focuses upon connecting specific Perceiver facts with specific Server sequences. This combination leads in concrete thought to a sense of cause-and-effect as Perceiver facts are connected with Server actions, and in abstract thought to precise meaning as Perceiver facts are connected with Server sequences of words.

In a similar manner, Exhorter connects Mercy with Teacher. Mercy thought applies emotional labels to experiential memories, leading among other things to a strong social awareness. Teacher thought, in contrast, comes up with general theories, which are usually communicated using words. Exhorter thought learns lessons from life, deriving general theories from personal experiences. Going the other 
way, Exhorter thought also applies theories to the emotional experiences of life. This relationship between experience and theory can be seen in intuition.

The combination of simple styles and composite styles provides a possible explanation for the partial brain lateralization that is observed in neurology: on the one hand, the left and right hemispheres use different strategies; but on the other hand, there is also extensive interaction between the two hemispheres. In a similar manner, the simple styles emphasize circuits within each hemisphere, while the composite styles build connections between these specific circuits.

That brings us to the question of cognitive style itself. What exactly is cognitive style? This question first arose back in the 1980s when I was helping my brother (Lane Friesen) analyze the data that he had collected from biographies. We realized that the personality traits of the seven cognitive styles could be summarized by fundamental modes of processing and that these fundamental characteristics mapped on to brain regions. But everyone has a complete brain. How can a person have a complete brain while also having a cognitive style that emphasizes part of the brain? Going further, I am not aware of any physical brain differences that would correspond to cognitive style. We concluded that cognitive style must reflect conscious awareness. On the one hand, every cognitive style has a similar brain which contains all seven cognitive modules. But on the other hand, several decades of observation have convinced me that different cognitive styles really do view the world in different ways; each cognitive style finds certain forms of thinking blindingly obvious, while other cognitive styles appear to be mentally oblivious to these same forms of thought.

I do not know what consciousness is-and I am not aware of anyone who knows what consciousness is, but I suggest that it involves the two traits of control and awareness. First, a person can choose either to use or not use a cognitive module that is conscious, regardless of the content within that mode of thought. In contrast, a cognitive module that is subconscious will only begin to function when the level of content reaches a certain threshold, and it is difficult to shut down a subconscious cognitive module that has started to operate. Second, a person is aware of the details of conscious thought, but will only notice the results of subconscious processing.

Thus, each cognitive style provides a window into the functioning of part of the brain. This means that it is possible to assemble a fairly complete picture of how the entire brain functions by examining how

each cognitive style deals with conscious thought and then putting these various pieces together guided by the diagram of mental symmetry. This summarizes the methodology that was initially used by mental symmetry to analyze the traits that were gathered from biographies.

The rest of the paper will look at various regions of the brain, starting with the orbitofrontal cortex. We will examine all the areas that are associated with higher level processing, but will not discuss any sensory, motor, memory, or autonomic regions.

\section{Orbitofrontal Cortex}

The orbitofrontal cortex is heavily interconnected with the amygdala and with the frontal pole of the

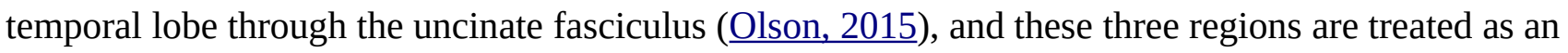
integrated system. Mental symmetry relates the left side to Teacher thought and the right side to Mercy thought. The orbitofrontal cortex will be discussed here, while the amygdala will be discussed later. 
Mercy thought adds emotional labels to experiences. Similarly, a recent paper on the orbitofrontal cortex concludes that "Research has shown that pleasure is never merely a sensation nor a thought, but an additional hedonic gloss, which is the pleasure versus displeasure affect that is actively generated by the brain and attached to its sensory or cognitive object” (Kringelbach, 2017, p. 4). This emotional labeling happens within the orbitofrontal cortex: "The midanterior subregion of the orbitofrontal cortex has emerged as an apex of pleasure system, linking reward with hedonic experience. Within this region there is strong, consistent activity correlating with the hedonic experience of a range of pleasures" (p. 5). This adding of an emotional label to an experience goes beyond physical sensations of pain and pleasure: "Hedonic evaluation of pleasure valence is anatomically distinguishable from earlier sensory computations, for example, how taste identification in the primary gustatory cortex in anterior insula is separate from valence processing in higher order areas such as orbitofrontal cortex” (p. 4).

Turning now to another paper, human and monkey brains distinguish between physical sensations and internally generated Mercy emotions. In contrast, physical sensation is the same as emotion in a rat brain: "Indeed there may be no cortical area in rodents that is homologous to most of the primate including human orbitofrontal cortex. The primate including human orbitofrontal cortex (OFC) implements reward value. Value is not represented at earlier stages of processing in primates including humans. Invariant visual object recognition is used for many functions including memory formation, so perception is kept separate from emotion. In contrast, in rodents, value is represented even in the first taste relay in the brain, the nucleus of the solitary tract: there is no clear separation between perception and emotion. In rodents, even the taste pathways are connected differently, with subcortical connections bypassing the cortex (including orbitofrontal cortex) and making connections via a pontine taste area directly to the hypothalamus and amygdala” (Rolls, 2017a, p. 251). In humans, the level of neuron activity in the orbitofrontal cortex corresponds to the level of emotion that a person says that they are experiencing: "Orbitofrontal cortex activations in humans to these stimuli are linearly related to the subjectively reported pleasantness of stimuli (medially) or to their unpleasantness (laterally)” (p. 255). Mercy thought 'lives' within an internal world of emotional experiences, while Server thought-a different cognitive module-performs the actions that are guided by these emotional experiences. Consistent with this, "In the orbitofrontal cortex, the representation is of the value of stimuli, and actions are not represented. The value of very many different types of stimuli, events, or goals is represented separately at the neuronal level, providing the basis for choice between stimuli and the selection at later stages of processing of an appropriate action to obtain the chosen goal” (p. 12).

Continuing with the paper by Rolls, the emotional label that Mercy thought attaches to an experience depends upon the context. For instance, food tastes better when one is hungry: "In the human orbitofrontal cortex, we found a large decrease in the BOLD signal to a complex food (tomato juice vs. chocolate) fed to satiety... This sensory-specific decrease was related to the decrease in the subjective pleasantness of the food eaten to satiety” (p. 259). The Mercy person is very aware of smell. Similarly, "The subjective pleasantness or reward or affective value of odour is represented in the orbitofrontal cortex, in that feeding humans to satiety decreases the activation found to the odour of that food, and this effect is relatively specific to the food eaten in the meal” (p. 259). The Mercy person reads emotional clues from peoples' faces. Similarly, “In humans, there is a part of the orbitofrontal cortex that responds selectively in relation to face expression specifically when it indicates that behaviour should change, and this activation is error-related and occurs when the error neurons in the 
orbitofrontal cortex become active” (p. 261). Mercy persons can change their opinion about people and situations very quickly based upon emotional incidents: "In primates and humans, the orbitofrontal cortex implements one-trial rule-based reversal learning, and this is important in rapidly updating social behaviour. This is rapid updating of value-based representations” (p. 264). Mercy thought functions associatively. Every experience brings to mind memories of related experiences, and the way that Mercy thought feels about the current experience will be influenced by the emotional labels assigned to these related memories. "The sight of chocolate produced more activation in chocolate cravers than non-cravers in the medial orbitofrontal cortex and ventral striatum. For cravers vs non-cravers, a combination of a picture of chocolate with chocolate in the mouth produced a greater effect than the sum of the components (i.e. supralinearity) in the medial orbitofrontal cortex and pregenual cingulate cortex. Furthermore, the pleasantness ratings of the chocolate and chocolate-related stimuli had higher positive correlations with the fMRI BOLD signals in the pregenual cingulate cortex and medial orbitofrontal cortex in the cravers than in the non-cravers” (Rolls, 2019b, p. 16).

The immature Mercy person is emotionally dependent upon the environment, while the mature Mercy person becomes emotionally guided by an internal world that overrides the feelings of the present. Similarly, Rolls states that "in primates including humans the amygdala becomes overshadowed by the orbitofrontal cortex. The orbitofrontal cortex has a much more important role in the computation of reward and punishment value, as measured by effects of devaluation and goal-directed one-trial rulebased learning and reversal, and thereby in emotion, which shows major changes after damage to the orbitofrontal cortex" (Rolls, 2017a, p. 267). In other words, the amygdala is an emotional processor that can be internally guided by emotional labels stored within the orbitofrontal cortex. The relationship between orbitofrontal cortex and amygdala will be examined further when looking at the amygdala.

This means that the Mercy person can use emotional content within the internal world to read things into social situations which are not actually there, such as ascribing motivations to people which they did not actually mean. Consistent with this, one recent paper suggests that the orbitofrontal cortex uses emotional clues to come up with an internal model of the current state based upon partial information. "We describe a novel theory that posits that OFC's specific role in decision-making is to provide an upto-date representation of task-related information, called a state representation. This representation reflects a mapping between distinct task states and sensory as well as unobservable information. We summarize evidence supporting the existence of such state representations in rodent and human OFC and argue that forming these state representations provides a crucial scaffold that allows animals to efficiently perform decision making and reinforcement learning in high-dimensional and partially observable environments" (Schuck, 2017, p. 1). Looking at this in more detail, "This requirement for the state representation raises another problem: some variables do not have a one-to-one correspondence to the information the agent gets from its sensors. For example, the velocity related variables must be inferred by comparing past and current sensory inputs, and thus require memory. If states need to reflect information beyond what is accessible through current sensory input and there is uncertainty regarding their true underlying value, the states are called partially observable. Finally, not all aspects of the current sensory input are relevant. Lighting conditions, for instance, do not need to be included into the state as they are irrelevant for the policy even if they change the sensory signals” (p. 5). 
Translating this into cognitive language, the Mercy person will come up with an internal emotional model of the current situation by focusing upon certain features and jumping to conclusions based upon partial information. This usually works well. It occasionally fails-often dramatically. Thus, emotions can be viewed as a shortcut for careful thought, a way of responding to a situation quickly and efficiently. Emotions are often viewed as the opposite of rational thought. But emotions can be trained to reflect rational thought, and this retraining of emotions will be discussed later, when looking at cognitive reappraisal.

The orbitofrontal cortex appears to be critical for internally representing some state with its emotional labels. However, most of the underlying calculations are being done elsewhere in the brain using a larger circuit that deals with what is known as Theory of Mind. Similarly, the Mercy person is keenly aware of the emotional state of a person or situation, but is much less talented when it comes to analyzing the details of some emotional state or working out how one gets from one emotional state to another.

Before we go on, I should emphasize that everyone uses this kind of processing to some extent. But the Mercy person appears to be conscious in this form of processing. Thus, observing what comes naturally to a Mercy person provides a window into what is happening within the orbitofrontal cortex. This does not mean that the Mercy person behaves only in this way. However, one will notice these traits protruding in the Mercy person in a manner that they do not with other cognitive styles.

I should also emphasize that no cognitive style is better or worse than the others. All cognitive styles have both inherent strengths and weaknesses. The goal for everyone, regardless of cognitive style, is to get all seven cognitive modules to function together in harmony, and much of my research on mental symmetry has focused upon this quest of reaching mental wholeness. However, that is not the primary topic of this paper, which is examining the correspondence between behavioral traits described by mental symmetry and the functions of brain regions.

\section{Teacher Emotion}

The average person equates emotion with Mercy emotion, regarding emotion as a label that the mind attaches to experiences, people, and situations. We have seen that this kind of emotional labeling happens within the orbitofrontal cortex: "Activations in the orbitofrontal cortex correlate with the subjective emotional experience of affective stimuli, and damage to the orbitofrontal cortex impairs emotion-related learning, emotional behaviour, and subjective affective state” (Rolls, 2019b, p. 1). In contrast to this general awareness of Mercy emotion, I have found that most people are unaware of Teacher emotion and instinctively reject the concept that Teacher thought functions emotionally.

I mentioned in the introduction that scientific thought always evaluates information in the light of some paradigm. This leads to curious results when neurology attempts to study the part of the brain that evaluates paradigms. That is because a paradigm functions emotionally, and mental symmetry suggests that this emotion is Teacher emotion. Stated as clearly as possible, what appears to happen is that if the paradigm of the researcher does not include an explanation for paradigms, then the researcher will be emotionally driven by that paradigm to deny the existence of a brain region that evaluates paradigms. The fundamental dilemma is that the mind is studying itself; neurologists are using their minds to study how the mind functions. This is an obvious statement, but it is seldom mentioned in neurological 
papers. Science typically addresses the problem of Mercy emotions by remaining objective. But how can one eliminate Teacher emotions if these are the very emotions that are driving a researcher to perform research? And what happens when one is being driven by Teacher emotions to study how the brain generates Teacher emotions?

Mercy emotions are easy to recognize but difficult to define. Teacher emotions, in contrast, can be defined quite simply. Teacher emotion is related to order-within-complexity; Teacher thought feels good when many items fit together in a simple manner; Teacher thought feels bad when there is an exception to the general rule. This kind of emotion is described in some recent papers.

A 2017 paper by Kringelbach and Berridge contrasts normal emotions with an emotion of well-being which they refer to as eudaimonia: "Since Aristotle, happiness has been thought of as consisting of the dual aspect of hedonia (pleasure) and eudaimonia (a life well-lived, embedded in meaningful values). Pleasure might be much easier to evoke but is often but a brief moment in happiness or in states of subjective well-being. Yet, it is clear that hedonia and eudaimonia empirically cohere together in happy people.” (Kringelbach, 2017, p. 6). They suggest that eudaimonia may be related to metastability: "It has been shown that when metastability is optimal, the system is best able to explore the dynamic repertoire, that is, an optimal point is reached between the fast and slow processing characterizing human cognition. Given these important results, we hypothesize as one possibility that such optimal metastability could be linked to a state of eudaimonia. In this brain state, there would be optimal flow of information in the pleasure system and connected emotion processing networks, which could correspond to the feelings of subjective well-being and flow anecdotally reported after a deeply meaningful experience of positive emotion” (p. 8). Hedonia describes Mercy emotions, which depend upon specific experiences. Eudaimonia with its metastability, in contrast, is an expression of Teacher order-within-complexity. On the one hand, there is order because everything fits together in a stable manner. But on the other hand, there is also complexity, because the order is continually expressing itself in new ways. This combination describes order-within-complexity, a dynamic stability in which everything remains integrated while continuing to change. Eudaimonia is also related to the concept of mental wholeness that was mentioned earlier, which was defined as all seven cognitive modules functioning together in harmony. This is also an example of order-within-complexity, and I mentioned that much of my thinking about the theory of mental symmetry has focused upon this task of achieving mental wholeness.

Achieving mental wholeness is significant from a personal perspective. If it is pleasurable to drive a car that is well-maintained and running smoothly, then presumably it would also be pleasurable to have a mind that is well-maintained and running smoothly. But pursuing a paradigm of mental wholeness is also significant from the perspective of Teacher emotion, because one is using a paradigm that emphasizes following paradigms; one is being driven by Teacher emotion to recognize Teacher emotion.

Beauty is another example of Teacher order-within-complexity, because a feeling of beauty emerges when many elements fit together in a harmonious manner. Research has recently discovered that the orbitofrontal cortex is sensitive to the emotion of beauty: "Brain imaging studies exploring the neurobiology of beauty have shown that the experience of visual, musical, and moral beauty all correlate with activity in a specific part of the emotional brain, field A1 of the medial orbito-frontal 
cortex, which probably includes segments of Brodmann Areas (BA) 10, 12 and 32” (Zeki, 2014, p. 1). Zeki has published several papers on the various forms of beauty that activate this region of the orbitofrontal cortex. (This location is on the boundary between orbitofrontal and medial frontal.)

Rolls describes this attribute of order-within-complexity in a paper on aesthetics: “A theory of the neurobiological foundations of aesthetics and art is described. This has its roots in emotion, in which what is pleasant or unpleasant, a reward or punisher, is the result of an evolutionary process in which genes define the (pleasant or unpleasant) goals for action. To this is added the operation of the reasoning, syntactic, brain system which evolved to help solve difficult, multistep, problems, and the use of which is encouraged by pleasant feelings when elegant, simple, and hence aesthetic solutions are found that are advantageous because they are parsimonious, and follow Occam's Razor” (Rolls, 2017b, p. 121). Notice the various adjectives describing order-within-complexity: elegant, simple, parsimonious, Occam's razor. Notice also that Rolls is presenting 'a theory' which 'has its roots in emotion'. Thus, Rolls is using his mind to evaluate a theory about how minds evaluate theories.

As another neurological paper points out, beauty can be viewed either as something that is natural, or as a social construct: 'According to aesthetic theories, a sense of beauty may be mainly affected by two factors. The first is objective parameters and external morphology of concrete objects, consistent with the perspectives of Plato's objectivist view of aesthetic perception. Previous studies have found symmetrical human faces, geometrical shapes and websites designs, symmetrical sequences of apparent movements, representational artworks, sculptures obeying the canonical proportion of the golden ratio, and paintings and polygon patterns with intermediate complexity, typically elicit higher aesthetic appraisal and experience, providing support of this theory that beauty has corresponding morphological characteristics” (Zhang, 2017, p. 1). Notice that all of these examples of beauty result from orderwithin-complexity that emerges in natural ways, such as symmetry or proportion.

Continuing with this paper, “An alternative theory argues that the perception of beauty is mainly based on the subjective construction of valence based on abstract social meaning and values. Prior studies have found smiling human faces with direct eye gaze, people with good inner character, and short sentences and scenes describing morally-positive actions can elicit a sense of beauty and produce enhanced activation of aesthetic reward systems due to the positive social meanings associated with these stimuli. These studies imply that aesthetic evaluation is a subjective construction and support the 'what is good is beautiful' theory” (p. 1). One possible interpretation is that social beauty is also an example of Teacher order-within-complexity in which Teacher thought is attempting to come up with a simple explanation for social facts and feeling good when it succeeds.

Looking more generally at these two kinds of beauty, everyone will tend to have similar concepts of objective beauty, because everyone is observing the same physical objects. In contrast, concepts of social beauty will vary between one culture or person and another, because each person is using Teacher thought to try to bring order to the complexity of facts that exist within that person's mind. Going the other way, when a person maintains that some facet of personality is 'too complicated to be summarized by any simple explanation', then this is really asserting that there is no underlying Teacher order-within-complexity. When a person states this as a general principle, then this promotes the general Teacher theory that there is no general Teacher theory. This is psychologically damaging because it sets Teacher thought against itself. 
Mathematical beauty is unusual. It appears to be an example of social beauty, because mathematical beauty is only recognized and appreciated by people who have studied mathematics. (A similar contrast can be seen to a lesser extent with visual beauty, because trained architects show greater activity in the

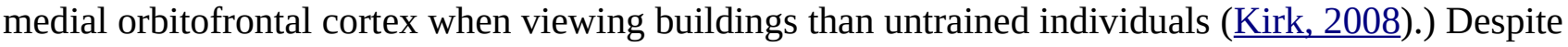
this, mathematical feelings of beauty are independent of culture: "The experience of mathematical beauty is perhaps the most extreme aesthetic experience that is dependent upon culture and learning; those not versed in the language of mathematics cannot experience the beauty of a mathematical formulation. And yet, once the language of mathematics is mastered, the same formulae can be experienced as beautiful by mathematicians belonging to different races and cultures” (Zeki, 2018, p. 7).

Zeki concludes that mathematical beauty is based in cognitive structure, and that people from different cultures have similar concepts of mathematical beauty because they have similar minds: "It is in this sense that mathematical beauty has its roots in a biologically inherited logical-deductive system that is similar for all brains. It is only by adhering to the rules of the brain's logical deductive system that a formulation can gain universal assent and be found beautiful” (p. 7). This describes the approach taken by mental symmetry. If similar patterns of behavior can be found in widely divergent specializations and cultures, then this is a strong indication that one is dealing with a cognitive mechanism.

However, when it comes to making a Teacher theory about Teacher theories, Zeki steps back and defines Teacher emotion using circular reasoning: "Beauty is an experience that correlates quantitatively with neural activity in a specific part of the emotional brain, namely, in the field A1mOFC; the more intense the declared experience of beauty, the more intense the neural activity there” (Zeki, 2019, p. 109). Zeki initially discovered that a specific region within the orbitofrontal cortex appreciates all kinds of beauty, and he is now defining beauty as something that activates this region of the orbitofrontal cortex.

When the mind is constructing a theory about how the mind constructs theories, then one must also analyze the theory that is within the mind of the researcher. This is easy to do in the case of Zeki because he devotes the opening third of his 2019 paper to a description of his theory. He begins by saying that "There have been many definitions of beauty, but none of them is wholly or even partially satisfactory. My favourite is the one given by Edmund Burke, partly because it says nothing and partly because it says everything” (p. 107). Zeki then continues by saying that beauty "is not, according to him [Burke], to be sought in proportion or in perfection, regardless of whether one is considering artefactual beauty, as in buildings, or biological beauty, as in human bodies” (p. 107). Zeki adds that "Nor is beauty to be found, according to Burke, in mathematically determined proportions because 'we begin to feel that mathematical ideas are not the true measures of beauty'” (pp. 107-108). However, he recognizes that "This dismissal of proportion and perfection would no doubt shock many today" (p. 107). But what empirical evidence does Zeki give for his dismissal of thousands of years of careful thought about beauty and aesthetics? None. He simply opens his paper by asserting that "There have been many definitions of beauty, but none of them is wholly or even partially satisfactory” (p. 107). And then he proposes a definition of beauty that says both nothing and everything. Thus, one sees that attempting to come up with a theory about theories is a cognitively perilous task, even for a welltrained neurologist. 
Rolls also uses strange reasoning when discussing Teacher emotion. In his paper on aesthetics, he explicitly states that the mind finds emotional pleasure in order-within-complexity: "Solving difficult problems feels good, and we often speak about elegant (and beautiful) solutions. What is the origin of the pleasure we obtain from elegant ideas, what makes them aesthetically pleasing? It is suggested that solving problems should feel good to us, to make us keep trying, as being able to solve difficult problems that require syntactic operations may have survival value. But what is it that makes simple ideas and solutions (those with fewest premises, fewest steps to the solution, and fewest exceptions for a given level of complexity of a problem) particularly aesthetically pleasing, so much so that physicists may use this as a guide to their thinking? It is suggested that the human brain has become adapted to find simple solutions aesthetically pleasing because they are more likely to be correct” (Rolls, 2017b, p. 130). Notice all the terms that refer to Teacher order-within-complexity: elegant, beautiful, simple ideas, fewest premises, fewest steps, fewest exceptions. Notice also the repeated references to emotion: feels good, pleasure, pleasing, feel good, pleasing.

But instead of recognizing that Teacher emotion is being generated by Teacher thought, Rolls bases his explanation of Teacher emotion in the Teacher theory of evolution: "When we understand the underlying origins and bases of aesthetics, we see that the processes involved are elegant and beautiful, as part of a Darwinian theory. (The processes involved in Darwinian evolution are elegant and beautiful, in that an efficient way to search a high dimensional space with multiple peaks of optimality is to use recombination of gene complexes produced by sexual reproduction to implement local hillclimbing in the space, and occasional mutations to produce a jump to a random position in the space to search for local optima there)” (p. 124). But what is causing Rolls to find emotional pleasure in Darwinian theory? Some part of his brain must be generating emotional pleasure from the concept that 'the processes involved in Darwinian evolution are elegant and beautiful'. However, instead of focusing upon Teacher emotions being generated by the brain, Rolls gets sidetracked by the Teacher emotions being generated in his brain by the theory of evolution. Again, one observes that coming up with a theory about theories is a cognitively perilous task.

I should mention before continuing that I have tried several times over the years to share the idea of Teacher emotion with other researchers, and I have consistently run into cognitive barriers. Thus, the papers on Teacher emotion that we have examined should not be viewed as exceptions to the rule, but rather as illustrations of a general cognitive hazard, and the authors are to be applauded for venturing into dangerous cognitive territory. I learned about Teacher emotion during the initial research that was done on mental symmetry, because I, a Perceiver person, was interacting extensively with my brother (Lane Friesen), who is a Teacher person. Because these two cognitive styles define abstract thought, this combination is very powerful for developing a general theory-if one can handle the emotional discomfort. In essence, one is functioning within Thomas Kuhn's realm of revolutionary science, in which paradigms are continually being proposed, tested, and rejected.

We have looked at Mercy emotion and Teacher emotion in isolation. Teacher thought can interact with Mercy thought in strange ways. For instance, it is possible for a person to find order-within-complexity in personal misery. The end result is that Teacher thought will feel good whenever Mercy thought feels bad, because personal misery provides evidence for a general theory of personal misery. Whenever 
Mercy thought feels good, then this pleasant experience will provide a counterexample to the general Teacher theory, making Teacher thought feel bad.

This combination can be seen in the 'hopelessness theory' of depression, which is summarized in the following quote: “According to Rose and Abramson, when confronted with a negative life event, the child attempts to come to an understanding about the cause of the maltreatment event, so as to prevent its potential recurrence. Initially, the child may be likely to form relatively benign causal attributions that are external, unstable, and specific in nature (e.g., one's parent was having a bad day). If these events become chronic or pervasive, however, such attributions are repeatedly disconfirmed, increasing the likelihood that the child will instead turn to more depressogenic causal attributions that are internal, stable, and global in nature (e.g., concluding that there is something inherent in oneself that makes one the target of repeated abuse from others)... childhood emotional abuse, in particular, has a deleterious effect on the child's cognitive style because, unlike in the case of childhood physical and sexual abuse, the abuse perpetrator provides the negative causal attributions directly to the child (e.g., 'you are so stupid, you will never amount to anything')” (Liu, 2015, p. 3). Notice that Teacher thought is coming up with a general theory which predicts in some way that Mercy thought will feel bad. The end result is that Teacher emotion will drive a person to continue ruminating about personal inadequacies within Mercy thought, because they provide data that confirm the general theory: "Depression is associated with increased attention to the self. Self-focus in depression is not only quantitatively increased, but also qualitatively distinct, characterized by repetitive thinking on negative aspects of one's self. This negative self-focus, or rumination, is strongly associated with vulnerability for depression” (Nejad, 2019, p. 37).

The problem is not that Teacher thought is being used, but rather that is being used in a psychologically harmful manner: "While brooding rumination may be associated with atypical emotion regulation in MDD [major depressive disorder], it has been suggested that rumination may take a more adaptive form in healthy non-depressed individuals. In particular 'reflective pondering' - a type of rumination with an emphasis on problem solving and addressing or alleviating negative emotions - is more prevalent than brooding rumination in healthy individuals. Further, unlike brooding rumination, it is not associated with depressive measures in healthy individuals. In contrast, in MDD brooding rumination is more prevalent than reflective pondering and both types of rumination are associated with increased depression” (Murphy, 2016, p. 91). Summarizing, 'reflective pondering' is psychologically healthy because one is using Teacher thought to understand how one could solve personal problems. In contrast, 'brooding rumination' finds Teacher order-within-complexity in one's state of personal misery. The first contains a sense of sequence and time, the second does not. We will return to this distinction when discussing Server thought.

\section{Inferior Frontal Cortex}

I have suggested that Teacher emotion comes from order-within-complexity-when many items fit together in a simple manner. There are many forms of order-within-complexity, but the Teacher person is attracted to words, and Teacher thought typically generates order-within-complexity by using words to come up with simple verbal theories. Teacher thought comes up with a theory by picking some single item within the current context and then treating that single item as if it is a general theory. One 
could compare this to taking a commoner off the street, crowning the commoner as monarch, and then seeing how well this ex-commoner functions as a monarch.

Research into the localization of brain function began with the discovery of Broca's area back in 1865. Paul Broca discovered that the left inferior frontal cortex was responsible for generating speech. Words and speech form the basic building blocks for Teacher thought. Thus, it makes sense to associate the left inferior frontal cortex with Teacher thought.

Modern research has found that the left inferior frontal cortex performs Teacher thought, in which one specific item is pulled out from a context of similar items: "Multiple lines of evidence support the involvement of left vlPFC in the cognitive control of memory" (Nyhus, 2015, p. 134). "According to the two-process model, mid-vlPFC is activated under conditions in which multiple items are retrieved from memory, but only a subset must be selected for further processing. As described above, automatic and controlled retrieval processes can result in the recovery of multiple representations. Thus, postretrieval selection is needed to resolve competition among the multiple retrieved representations, and to permit selected representations to guide decision and action" (pp. 135-136). "In summary, there is evidence both across and within studies for dissociable functions between anterior vlPFC and mid vlPFC during cognitive control of memory, and these functions can be characterized as controlled retrieval and post-retrieval selection respectively" (p. 137). (vlPFC stands for ventral lateral prefrontal cortex and is another name for inferior frontal cortex.) In other words, the middle part of the left inferior frontal cortex creates a context by retrieving several related items. The front part of the left inferior frontal cortex then picks one of these items from the context.

This also explains how Teacher thought views a general theory. The Teacher person often claims to be searching for universal theories. But this is not entirely accurate. Instead, what the Teacher person really wants is to have a general theory and not be aware of any exceptions to this general theory. In other words, the general theory does not have to explain everything. Instead, it only has to explain everything within the context. Using an analogy, a general Teacher theory is like some tribal chief who proclaims that he is ruler over the entire world. The chief can maintain this claim as long as he is not aware of any other people living outside of the small valley over which he rules. Similarly, Teacher thought can maintain the illusion of a general theory by 'pulling up mental drawbridges' and ensuring that contradictory facts never enter into the 'castle' or domain of the theory.

This principle can be seen when comparing children who speak one language with those who speak several languages: “Children in a multilingual environment exhibited stronger functional connectivity between the left pars triangularis seed region and a large cluster spanning the bilateral superior parietal lobule (SPL), compared to children in the monolingual group. Children in a monolingual environment displayed stronger connectivity between the left pars triangularis and the inferior and middle temporal gyri" (Thieba 2018, p. 10). [The pars triangularis is another name for the mid-vlPFC.] In other words, if one speaks only one language, then it is sufficient to use Teacher thought to retrieve the appropriate words from verbal memory within the temporal lobes. But if one speaks several languages, then Perceiver and Server connections are required to connect one verbal context from another in order to ensure that the appropriate language context is being used. 
Looking at this from a different perspective, Teacher thought plays a critical role in inductive reasoning, in which one comes up with a general explanation to explain many similar situations. The left inferior frontal cortex is connected with inductive reasoning: "Our data suggest that the left vlPFC contains populations of neurons specifically tuned to process domain-dependent information required to carry out inductive reasoning tasks, by creating domain-based rule representations that may dynamically change across sessions” (Furlan, 2018, p. 16). Notice that this inductive reasoning can 'dynamically change across sessions', consistent with the idea that Teacher thought comes up with a general theory that applies to the current context.

Mental symmetry suggests that Teacher thought emphasizes abstract reasoning rather than concrete experiences. This distinction is backed up by neurology: "Both abstract and concrete concepts, however, conjointly rely on perceptual, verbal and contextual knowledge, with abstract concepts characterized by low values of imageability (IMG) (low sensory-motor grounding) and low context availability (CA) (more difficult to contextualize). Imaging studies supporting differences between abstract and concrete concepts show a greater recruitment of the left inferior frontal gyrus (LIFG) for abstract concepts... The LIFG was significantly more activated for abstract than for concrete words, and a conjunction analysis showed a common activation for words with low IMG or low CA only in the LIFG, in the same area reported for abstract words” (Della Rosa, 2018, p. 449).

Moving on, mental symmetry suggests that the right inferior frontal cortex is related to Mercy thought. This region has traditionally been associated with the non-verbal aspects of speech, and the Mercy person tends to be more sensitive to the way in which a person says something than to the actual words being spoken. However, recent research has come up with a more refined understanding. "More specifically, dysfunction of intrinsic (also called linguistic) features of prosody (such as stress, rhythm and pitch) seem to result from damage to left inferior frontal regions, whereas lesions to right inferior cortex often results in impaired processing of extrinsic features of speech like affective prosody - the tonal variation conveying emotions” (Kellmeyer 2016, p. 17). Thus, both Teacher thought and Mercy thought function emotionally. But Teacher emotion is related to the structure of language, whereas Mercy emotion comes from the situation about which one is talking.

The right inferior frontal cortex carries out a non-verbal version of the kind of processing that is performed by the left inferior frontal cortex. For instance, the right inferior frontal cortex is sensitive to exceptions to a general rule of music: "A dependency is said to be local if it relates elements that directly follow one another in a sequence, and non-local if the dependency spans over multiple intervening elements" (Cheung, 2018, p. 1). "We found that violations to non-local dependencies in nested sequences of three-tone musical motifs in musicians elicited increased activity in the right IFG. This is in contrast to similar studies in language which typically report the left IFG in processing grammatical syntax” (p. 1). Notice that this study is not examining large-scale musical structure that covers many contexts, but rather looking for a simple three-tone pattern within the current musical context. This study also noted that developing musical skill leads to long-term structural changes in the right inferior frontal: "The participants of our study were trained musicians - a group shown to exhibit increased grey-matter volume in the right IFG” (p. 9).

The right inferior frontal cortex has traditionally been connected with 'response inhibition'. And Mercy thought often functions in this manner within modern Western society, stepping in to inhibit responses 
when they violate the cultural and personal expectations of Mercy thought. More recent research divides this region into an area that inhibits responses and one that detects relevant information. There is "strong evidence that the ventral portion of the rIFC (i.e., rIFG) is substantially involved in response inhibition but does not primarily serve the purpose of attentional control, or the integration of attended stimulus features with task goals... In contrast, activity of the dorsal portion of the rIFC (i.e., rIFJ) was linked to attention as it was associated with the detection of salient stimuli” (Sebastian, 2016, p. 12). (rIFC is right inferior frontal cortex.) The authors explain that "The flexible and favorable adaptation of our behavior to changing situations or environments is an important factor of executive control. It is critically dependent on attention steering and motor control. In many circumstances, salient external signals capture our attention and indicate that our ongoing actions or response tendencies need to be stopped and updated. Response inhibition is required when the goals of our actions are no longer adequate or even harmful” (pp. 2-3). One can see this kind of processing in the Mercy person. Mercy thought will focus upon some external experience — or internal wish —and then interpret the environment in the light of this fixation. For instance, 'The light just turned red, we need to stop.' Or 'I want to buy the latest gadgets so that I can make an impression upon my friends'. In both cases, Mercy thought is fixating upon some experience and expecting concrete thought to be guided by this fixation. This is similar to the way that Teacher thought comes up with a general theory by treating some concept as a general theory that can be used to explain other concepts.

The Mercy person is very sensitive to the eyes. For instance, when I was a child my mother (a Mercy person) would repeatedly tell me that I should 'Look into their eyes and say thank you with expression.' (We will look at the eyes again when discussing the amygdala.) The Mercy person also wants close friends with whom emotional bonding is possible. A recent study scanned the brain activity of two individuals who were looking at each other's eyes (via cameras and video monitors). They found that "Eye contact and joint attention are tightly coupled to generate the state of shared attention across individuals. Hyperscanning fMRI conducted with pairs of adults during joint attention tasks showed interindividual neural synchronization in the right inferior frontal gyrus” (Sadato 2017, p. 207). Looking at this cognitively, people who stare into each other's eyes become mutually guided by a single focus of attention in Mercy thought, and this generates positive Mercy emotions.

Stating this more generally, the right inferior frontal cortex is related to feelings of empathy. One study describes "the significant activation of the right IFG, right INS and right amygdala in children during engagement in empathic behavior and showed that the activation of the right IFG is significantly associated with the Interpersonal Reactivity Index (IRI), a self-reported empathy questionnaire” (Wu, 2018, p. 2). And this study found that electrically stimulating this region enhances measures of empathy while inhibiting it reduces measures of empathy. (This is done by applying electrodes to the scalp which deliver a small direct current.)

\section{Dorsolateral Prefrontal Cortex}

My hypothesis is that the left dorsolateral prefrontal is related to Server thought and the right dorsolateral prefrontal to Perceiver thought. The classic way of testing for damage in this part of the brain is the Wisconsin Card Sorting Test, and "data suggest that the functional integrity of the right dorsolateral frontal-subcortical circuit is critical for WCST performance” (Lombardi, 1999, p. 2). The 
patient is given a set of playing cards, each of which contains a certain number of shapes with different color and shape. The patient is then told to sort the cards but not instructed whether to sort them by number, color, or shape. Instead, he is simply given a feedback of right or wrong. Without telling him, the tester will change the sorting method several times through the test. A person with dorsolateral frontal damage will continue with his sorting strategy and not change to a new one. He may say, "I know that you are now sorting the cards by shape,” and yet continue to sort them by color.

This describes the function of Perceiver thought. Perceiver thought works out the facts, holds on to them as truth, and then uses this truth to shape behavior. Similarly, internal Server thought works out what to do, holds on to it, and then carries out this action. Talking about a plan involves Teacher thought. Carrying it out requires Server thought.

The dorsolateral prefrontal cortex is active when learning about connections between items, but not when learning about individual items themselves. This is consistent with the suggestion that Perceiver and Server thought look for connections between items. "Studies of memory encoding have reported DLPFC activation during interactive imagery of pairs of words, during intentional encoding of pairs of images, during reordering of word triplets based on a semantic rule, and while subjects judged the number of semantic associations present among a list of three words. All of these findings share a common basic principle: DLPFC activation was obtained in tasks that involved active processing of relationships between items that were active in working memory” (Murray, 2007, p. 5520).

Quoting from a more recent paper, “The dlPFC has been implicated in various aspects of domaingeneral reasoning: executive functioning, cognitive control, goal-directed planning, cost-benefit analysis, problem solving, counterfactual reasoning, [and] model-based control” (Patil, 2018, p. 7). Summarizing, "There is convergent evidence from functional and structural MRI studies to implicate dlPFC in domain-general reasoning in both moral and non-moral domains” (p. 8). These various functions all describe a mind being guided by Perceiver facts and Server sequences. Mental symmetry suggests that Perceiver facts and Server sequences can be overwhelmed by emotional pressure. This interplay between knowledge and emotions can be seen in the following quote from the same paper: "In the domain of moral judgment and decision-making, the dlPFC has been associated with the ability to- (i) facilitate abstract reasoning (e.g., cost-benefit analyses), and (ii) provide cognitive control to override strong social-emotional responses elicited by the aversive nature of moral dilemmas” (p. 7).

I have suggested that Perceiver thought is related to the right dorsolateral frontal cortex. Perceiver thought organizes Mercy experiences into categories by looking for connections that do not change. The right dorsolateral frontal plays a major role in maintaining honesty: "Honesty can be increased in humans with transcranial direct current stimulation (tDCS) over the right dorsolateral prefrontal cortex" (Maréchal, 2017, p. 4360). Honesty can be defined cognitively as recognizing which connections are fixed and do not change.

Stated more generally, the right dorsolateral frontal cortex evaluates beliefs: "According to the TwoFactor theory of delusional belief, there exists a cognitive system dedicated to the generation, evaluation, and acceptance or rejection of beliefs. Studies of the neuropsychology of delusion provide evidence that this system is neurally realized in right dorsolateral prefrontal cortex (rDLPFC)” (Coltheart, 2018, p. 1). The Perceiver person naturally emphasizes truth and beliefs. 
The right dorsolateral frontal cortex suppresses emotional urges: "Individuals displayed significantly riskier decision-making after disruption of the right, but not the left, DLPFC. Our findings suggest that the right DLPFC plays a crucial role in the suppression of superficially seductive options” (Knoch, 2006, p. 127). Similarly, a recent paper concluded that "Our results suggest that the right DLPFC is a valid target for manipulating risky behavior in tasks with a motor-response. Following rTMS stimulation participants' preference shifts toward immediate awards, while becoming significantly less sensitive to potential negative consequences. The results confirm that the right DLPFC is involved in impulse control in goal-directed executive tasks” (Tulviste, 2019, p. 72). I mentioned earlier that confidence can become overwhelmed by emotions. Thus, the Perceiver person naturally suppresses emotional urges in order to defend conscious thought from emotional attack. It is possible to increase confidence by successfully holding on to Perceiver facts (or Server sequences) in the midst of emotional pressure, but this strategy still involves not giving in to emotional urges. Going further, the categorizing done by Perceiver thought naturally limits the wishful thinking that tends to be present in risky behavior, because it forces a person to be honest about the facts and risks.

The right dorsolateral frontal is not needed to come up with moral rules, but it is required to implement these rules: "Disruption of right DLPFC activity only affects fairness-related behaviors but not fairness judgments. Subjects behave as if they can no longer implement their fairness goals after disruption of the right DLPFC” (Knoch, 2006, p. 832).

Another paper reviewed many studies of moral behavior and the right dorsolateral prefrontal cortex and came to the following conclusions: "Inhibition of the right DLPFC reduces the influence of harm on decision-making, both when deciding to perform a harmful action oneself, and when punishing harmful or unfair behaviors in others. This reduces decisions to punish or disapprove of potentially harmful personal moral violations in others. However, it also increased the likelihood of performing a harmful action oneself, such as lying or reacting aggressively. In contrast, excitatory brain stimulation to the right DLPFC reduced aggression and increased adherence to social norms. Taken together, these results suggest that the right DLPFC is important for representing aversion to harm, leading individuals to avoid harming others, but also to punish those who do cause harm” (Darby, 2017, p. 7). Saying this more simply, Perceiver thought leads to the rule of law. On the one hand, this protects personal identity in Mercy thought. But on the other hand, this leads to punishment for those who violate the law.

The right dorsolateral frontal does not just function morally but is also responsible for error-checking facts. One study "aimed to assess whether the application of tDCS over right DLPFC could modulate older adults' awareness of their performance errors. In two separate experiments, we demonstrated a reliable increase in the number of errors that were signaled by older adults during anodal stimulation to right DLPFC... We additionally demonstrated that, although anodal tDCS stimulation was effective when applied to right DLFPC, no change in performance was observed when left DLPFC was targeted. Our study therefore provides novel evidence that DLPFC regions subserving error awareness are predominantly lateralized to the right hemisphere.” (Harty, 2014, p. 3649). (Anodal tDCS electrically stimulates a brain region.) This describes another common trait of the Perceiver person, who is naturally talented at pointing out factual errors.

I mentioned earlier that the left inferior frontal cortex is associated with Teacher thought, because it retrieves some specific item from a context of similar items. I also mentioned that a theory will appear 
universal to Teacher thought as long as nothing within the current mental context contradicts that theory. When Perceiver thought error-checks facts, then this may cause contradictions to become apparent in Teacher theories. For instance, if one has been caught out in the rain several times during the last few weeks, Teacher thought may come up with the general theory that 'It always rains on weekends'. Perceiver thought may then deflate this theory by pointing out that 'It did not rain last weekend'. Teacher thought will then respond by either abandoning the theory or by limiting its domain. However, limiting the domain of a theory will reduce Teacher feelings of order-within-complexity. Using an analogy, the tribal chieftain now knows that he is only the head of some small tribe and not the ruler of the entire world.

Perceiver thought can also expand the domain of a Teacher theory by building connections between one context and another. A recent paper examined this relationship between forming a general theory and connecting general theories: “Participants' behavior suggested that they quickly discovered the most compact description of each sequence in a language comprising nested rules, and used these rules to compress the sequence in memory and predict the next items. Activity in dorsal inferior prefrontal cortex correlated with the amount of compression, while right dorsolateral prefrontal cortex encoded the presence of embedded structures” (Wang, 2019, p. 245). In other words, Teacher thought in the inferior frontal cortex is coming up with a theory that provides a 'compact description of each sequence' while Perceiver thought in right dorsolateral frontal cortex is expanding this theory by applying it to many different contexts.

We have seen that Perceiver thought focuses upon the facts, limits wishful thinking in Mercy thought, and points out errors in Teacher theories. This 'executive function' is essential when dealing with the physical world, because wishful thinking and mistaken theories tend to result in physical harm. However, if one lets go of Perceiver facts in a manner that does not affect one's grasp of physical reality, then it is possible to experience intense emotions. This provides a cognitive explanation for the ecstatic experience of mysticism.

The suppression of categories is a primary characteristic of mysticism: "According to this model, four main phenomenological features characterize mystical experiences (a breakdown in the usual sense of the passage of time; a breakdown in the usual sense of the extension of space; a breakdown in the differentiation between objects in the external world; and a breakdown in the differentiation between the self and the external world)" (Cristofori, 2015, p. 212). Cristofori suggests that mysticism is connected with a suppression of the dorsolateral prefrontal cortex: "Patients with focal lesions in the dIPFC had substantially higher mysticism scores compared to the controls. Collectively, these findings identify a homeostatic role of these brain regions with the dlPFC suppressing mystical experiences while regions within the temporal cortex, if unchecked, are more likely to produce mystical experience... we argue that the dlPFC has a critical role in regulating mystical experiences by binding context, deliberation, and rational experience to posterior cortices strongly affected by perceptual phenomena” (p. 217). “Our findings support previous speculation linking executive brain functions to mystical experiences, and reveal that executive functioning (dlPFC) causally contributes to the downregulation of mystical experiences” (p. 212).

Looking at this cognitively, mysticism combines overgeneralization in Teacher thought with identification in Mercy thought. The general theory that 'It always rains on weekends' mentioned 
earlier is an example of overgeneralization, and overgeneralization will naturally become limited by Perceiver facts, as illustrated by the response 'It did not rain last weekend'. Children use overgeneralization when learning grammar. Children will first overextend rules of grammar, using phrases such as 'I go-ed to the store' before learning to restrict the domain of their rules (Ambridge, 2013). Overgeneralization relates to Teacher emotion, because Teacher thought uses words to construct general theories, and an overgeneralized theory feels better than a restricted one. If Teacher emotion relates to generality, then the strongest Teacher emotions would come from a universal theory. This Teacher emotion can be seen in the quest within physics for a unified theory of everything.

The easiest way to form a universal theory is through overgeneralization. Teacher thought proposes the theory that 'All is One' and then protects this ultimate overgeneralization from Perceiver contradictions by asserting that this cosmic oneness transcends all facts of reality. In terms of an analogy that was used earlier, this is like crowning a commoner and then making sure that no one else shows up to challenge his rule. However, merely stating that 'All is One' is not enough, because there will be order but not complexity. But if one struggles with the complexities of life and then breaks through to the realization that 'All is One', then this will create strong Teacher feelings of order-within-complexity.

This transition from complexity to clarity can be seen especially clearly in the Zen koan, in which one struggles with a rational contradiction to the point of breaking through to a feeling of universality. In the words of one author, “According to a widely accepted model, a kōan is a clever psychological device designed to induce satori or kenshō. The koan is said to pose to the Zen practitioner a paradox unsolvable by the rational, intellectualizing mind. Driven into an ever more desperate corner by his repeated futile attempts to solve what cannot be rationally solved, the practitioner finally breaks through the barrier of rational intellection to the realm of preconceptual and prelinguistic consciousness variously called pure consciousness, no-mind, without-thinking, or emptiness. This breakthrough is called satori or kenshō” (Hori, 2000, p. 280).

This means that rational analysis of mysticism will naturally inhibit the experience of mysticism, and the mystic will usually warn against attempting to analyze mysticism. For instance, Hori responds to the widely accepted model of a Zen breakthrough with emotional derision: "These conceptions of kōan and kenshō were criticized long ago... 'People who utter such nonsense have not yet met a true mentor; hence they lack the eye of proper study. They are fools not worthy of mention... What these pseudoBuddhists regard as 'incomprehensible utterances' are incomprehensible only to them, not to buddhaancestors'. For Dogen, these 'pseudo-Buddhists' are merely rationalizing their ignorance. Not wishing to admit they have failed to comprehend enlightenment, they claim that enlightenment itself cannot be comprehended" (p. 281). He explains later in the chapter that "every attempt to understand non-duality as an interpretation reduces it to duality, since interpretations divide into dualistic categories like true/false” (p. 303).

Looking at this cognitively, mysticism is based upon the ultimate Teacher overgeneralization of universal oneness. Achieving this level of overgeneralization requires transcending all Perceiver facts, which means that any Perceiver facts will limit this ultimate Teacher overgeneralization-including Perceiver facts about universal oneness. Therefore, the non-mystic observing mysticism from the outside can analyze what is happening cognitively, as shown by the widely accepted model of a koan. However, the mystic must reject all such explanations because they will limit the practice of 
mysticism. This rejection cannot be done rationally, because using rational thought destroys the practice of mysticism. And a theory of oneness will only have a universal domain if overgeneralization is regarded as a superior form of thought that transcends rational thought. Therefore, rational explanations of mysticism must be rejected emotionally by calling analyzers fools who do not know how to think properly.

Mysticism then uses Mercy thought to identify with the overgeneralized theory of cosmic unity in Teacher thought. As Cristofori summarizes, "Mystical experiences can be defined as fusion with a supernatural agent... Mystical experiences are characterized by a sense of timelessness and union with a supernatural entity" (Cristofori, 2015, p. 212). As Cristofori states, mysticism is typically regarded as the essence of religion: "Mystical experiences, which can be defined as subjectively believed encounters with a supernatural being or supernormal world, are thought to underpin ordinary religious beliefs” (p. 212). Mental symmetry suggests that mystical tendencies will naturally emerge whenever one pursues Teacher theory in a subjective manner using vague facts and imprecise meanings. For instance, one can see a mystical flavor-accompanied by a rejection of rational analysis_in Zeki's definition of beauty quoted earlier.

If mysticism is the ultimate basis for all religion, then one concludes that religion is incompatible with rational thought. However, I suggest that this is not the case. That is because one can use the theory of mental symmetry to construct a rational Teacher understanding of religion based upon the order-withincomplexity of pursuing mental wholeness. This analysis has been summarized in two books (Friesen, 2012, 2015) and there is also extensive material on the mentalsymmetry website. Looking specifically at the topic of deity, my hypothesis is that a mental concept of God will emerge within the mind whenever a sufficiently general theory in Teacher thought applies to personal identity in Mercy thought. For instance, the mystic will not just feel that he had a transcendental experience. Instead, he will believe that he achieved personal union with God. However, there are other ways of constructing a general theory that applies to personal identity. For instance, a sufficiently general theory of mental wholeness will also lead eventually to a concept of God. But this concept of God will be quite different than the concept of God that emerges from mysticism.

Moving on, I have suggested that the left dorsolateral frontal cortex is related to Server thought. Server thought gives stability to sequences, including the sequences of Teacher thought. Electrically suppressing this region makes it more difficult to comprehend 'garden-path' sentences that have ambiguous interpretations, such as 'While the thief hid the jewelry sparkled brightly' (Hussey, 2015). This suggests that Server thought is not needed to comprehend speech, but it is needed to guide Teacher comprehension of speech along a certain path.

Looking at this more generally, the left dorsolateral frontal guides the sequence of thought: "Our study provides important evidence implicating left DLPFC in the process through which we appropriately prioritise the nature of ongoing thought in a context-dependent manner” (Turnbull, 2019, p. 7).

Electrically suppressing the left dorsolateral frontal leads to action that is more spontaneous and less planned. One group "hypothesized that cathodal tDCS over the left DLPFC area during motor learning would suppress the use of working memory and reduce explicit verbal-analytical involvement in movement control, thereby promoting implicit motor learning" (Zhu, 2015, p. 3). And they found "that 
cathodal tDCS over the left DLPFC promoted performance that was more implicit and automatic" (p. 7). (Cathodal tDCS electrically suppresses a brain region.) Zen Buddhism follows a similar path by associating mysticism with spontaneous action, which is consistent with the suggestion made earlier that mysticism functions by shutting down the 'executive function' of Perceiver and Server thought.

Depression is related to dysfunction of the left dorsolateral frontal: "Damage in the left dorsolateral prefrontal cortex is associated with greater depression symptoms.” (Grajny, 2016, p. 292). "Idiopathic major depression is associated with dysfunction in the left dorsolateral prefrontal cortex, as is latelife depression” (p. 292). Looking at this from a cognitive perspective, Server persons are seldom depressed. Instead, they usually function within an emotionally stable aura of pleasant contentment-as long as there is something that they can $d o$. However, the Server person will become emotionally unstable if faced with a problem for which nothing can be done in the present. Thus, it makes sense that enhancing Server thought would tend to lift a person out of depression.

Looking at this in more detail, I suggested earlier that one major source of depression is holding on to a general theory of personal misery, as described by the helplessness theory of depression. (Depression could also result from a lack of Teacher understanding, because there is no positive Teacher emotion to bias feelings in a positive direction.) One facet of helplessness is that nothing can be done-no Server sequences exist that could dislodge a person from their present state of misery. Server thought helps to unstick this mindset by suggesting the possibility of change.

Server sequences also impose a context upon Teacher thought. Thus, carrying out some plan will naturally move Teacher thought away from the context of personal misery to the context of the current Server sequence. Stated simply, if I am miserable, then it often helps to do something, because this will take my mind off my misery. Going further, doing something as part of a general plan will place my Server actions within a Teacher structure, making it possible for Teacher thought to find pleasure in being part of some greater plan.

I should point out that these cognitive interactions will happen regardless of whether a cognitive module is conscious or subconscious. However, when they happen subconsciously, then they will occur automatically outside of conscious awareness. For instance, the Mercy person can be prone to depression, because the Mercy person has no direct conscious control over how subconscious Teacher thought functions. Instead, brooding over unpleasant experiences in Mercy thought will indirectly cause subconscious Teacher thought to come up with a general theory of personal misery, and the Mercy person will also have to use indirect methods to escape from such depression.

\section{Frontopolar Cortex}

The region in front of the dorsolateral frontal cortex in the very front of the brain is called the frontopolar cortex. It was suggested back in 2000 "that dorsolateral prefrontal cortex is involved when externally generated information is being evaluated, whereas the frontopolar cortex becomes recruited when internally generated information needs to be evaluated” (Christoff, 2000, p. 168). This leads to the hypothesis that when Perceiver facts and Server sequences are internally driven, then the frontopolar region is required, whereas the dorsolateral frontal cortex is sufficient when Perceiver and Server thought manipulate external elements. 
More recent papers have clarified what this means. One author connects the functioning of frontopolar cortex with mental schema: "The most rostral zone includes RLPFC and represents a range of control signals that we term schematic control in order to convey its generality beyond only temporal or episodic signals. Bartlett coined the term schema as a knowledge structure that organizes many lower order features and their relationships. In essence, schemas are models of the world and ourselves in it" (Badre 2018, p. 10). The author summarizes that the "RLPFC is consistently engaged when control depends on an episodic or temporally structured context, integration and inference over multiple features, and when tracking hypothetical strategies, goals, and pending states. These types of control broadly depend on the structured information that schemas are proposed to hold” (p. 11). (RLPFC refers to rostral lateral prefrontal cortex, which is another name for frontopolar cortex.) Saying this cognitively, the frontopolar cortex is required when thought and behavior need to be guided by an internal framework of Perceiver facts and Server sequences.

This paper describes the standard model of viewing the frontal cortex as a series of increasingly abstract regions: "The cascade model proposed that hierarchical control is supported by a propagation of top-down control signals from rostral to caudal areas. RLPFC might form the apex of the frontal hierarchy and would influence mid-DLPFC, which would influence prePM, and so forth toward motor cortex" (p. 14). This implies that "any area higher in the hierarchy might exert asymmetrical influence, with broader efferent connections to lower order (i.e., caudal) areas than the reverse. Thus, if it is the apex, RLPFC should show the highest connectional asymmetry. However, among lateral PFC regions, anterior mid-DLPFC (areas 45 and 46) showed the greatest asymmetry, while RLPFC (area 10) was average on this metric” (p. 14). I am not certain if what the paper calls the mid-DLPFC includes aspects of the inferior frontal cortex, which relate to Mercy and Teacher thought. Whether this is the case or not, frontopolar cortex appears to provide the most general, cross-contextual, mental framework. But it does not seem to be the center of control. This may explain the way that a Perceiver person views truth. The Mercy person identifies with experiences and the Teacher person often identifies with his theories. In contrast, the Perceiver person seldom identifies with his truth, but rather views truth as something solid apart from conscious thought to which conscious thought submits.

Monkeys do not have a true frontopolar cortex: "Recordings in the most anterior part of the cortex in the macaque monkey have been sparse and have yielded results that differ substantially from what was predicted based on human neuroimaging studies, leading some authors to suggest that part of the human anterior prefrontal cortex might be uniquely human” (Hartogsveld, 2018, p. 2). Saying this cognitively, it appears that only humans are capable of being guided by internal schema that represent general principles which transcend specific situations. In the words of this paper, "non-human primates have difficulty with the fast processing of relations between cognitive representations that humans are capable of. The finding that the lateral frontal pole might not have a clear homolog in the macaque monkey brain and the current confirmation that this area overlaps with the region that is activated during relational processing suggest it might be involved in this human ability” (p. 10).

I suggest that the right frontopolar cortex is related to Perceiver thought. The Perceiver person has a strong need to populate his mental map of knowledge with new facts. Consistent with this, electrically suppressing the right frontopolar cortex inhibits directed exploration: "Using transcranial magnetic stimulation to inhibit the right frontopolar cortex, we were able to selectively inhibit directed 
exploration while leaving random exploration intact. This suggests a causal role for right frontopolar cortex in directed, but not random, exploration” (Zajkowski, 2017, p. 1). The Perceiver person builds a mental map upon truth — facts that are known with certainty. Activity in the right frontopolar cortex increases when facts are known with greater certainty: "We measured the brain activity by fMRI while healthy subjects performed a visual short-term recognition memory test and then rated their confidence in their answers as high, middle, or low... Among brain regions showing greater activity during rating their confidence relative to during a control, non-metamemory task (discriminating brightness of words), only a posterior-dorsal part of the right frontopolar cortex exhibited higher activity as the confidence level better correlated with actual recognition memory performance. These results suggest that activation in the right frontopolar cortex is key to a reliable, retrospective rating of confidence in short-term recognition memory performance.” (Yokoyama, 2010, p. 199).

The left frontopolar cortex appears to be critical for analogical reasoning: "We examined analogical reasoning abilities in 27 patients with focal damage in the frontal lobes and performed voxel-based lesion-behaviour mapping and tractography analyses to investigate the structures critical for analogical reasoning. The findings revealed that damage to the left rostrolateral prefrontal region (or some of its long-range connections) specifically impaired the ability to reason by analogies” (Urbanski, 2016, p. 1783). And electrically stimulating this region enhances creativity: "High definition tDCS-targeted frontopolar cortex activity [was] recently shown to predict state creativity augmentation. In a novel analogy finding task, participants under tDCS formulated substantially more creative analogical connections in a large matrix search space (creativity indexed via latent semantic analysis). Critically, increased analogical creativity was not due to diminished accuracy in discerning valid analogies, indicating 'real' creativity rather than inappropriate divergence. A simpler relational creativity paradigm (modified verb generation) revealed a tDCS-by-cue interaction; tDCS further enhanced creativity cue-related increases in semantic distance" (Green, 2017, p. 2628). (The diagram in this paper makes it clear that the left frontopolar cortex is being stimulated.)

I have suggested that the left frontopolar cortex is related to Server thought. However, the average Server person is not creative, but rather tends to repeat sequences of actions without varying them creatively. Extended observation back in the 1980s led my brother and I to conclude that the average Server person uses Server thought to perform concrete actions but seldom develops the abstract capabilities of Server thought. Saying this another way, Server thought is able to express itself through the sequences of physical action. When this is the primary way of expressing Server thought, then the structure of the external world will substitute for internal content. One can tell that internal content is lacking because the typical Server person finds it very disorienting to move from their existing home to live in a new location.

In contrast, I discovered as a Perceiver person that I was capable of using creative analogical reasoning when I stopped thinking in terms of static Perceiver facts and started thinking in terms of processes and temporal connections of cause-and-effect. Saying this cognitively, Perceiver thought gains access to Server thought as Contributor thought builds connections between Server and Perceiver. In fact, creative analogy is the primary form of thought that I use to expand the theory of mental symmetry. As Green points out in his paper, "Creativity research indicates that new creative ideas often take the form [of] connections between concepts that had previously seemed far apart. Relational cognition that 
reveals distant connections, especially in the form of analogical reasoning, is frequently a mechanism for generating creative leaps in the sciences, arts, education, and industry” (p. 2629). The primary feature of the theory of mental symmetry is that it 'forms connections between concepts that had previously seemed far apart.'

\section{Hippocampus}

My general hypothesis is that the left and right hippocampi are the processors for Server and Perceiver thought, in the sense that they determine the state of Server and Perceiver thought at the current moment. In the words of one researcher, "the hippocampus prominently exhibits activity reflecting the immediate experiences of the animal. This phenomenon has been robustly captured in the spatial domain within monkey hippocampal neurons, just as it has been often demonstrated in rodents” (Rueckemann, 2017, p. 158).

There is evidence supporting a lateralization, with Server using the left hippocampus and Perceiver the right. For instance, "Individuals with larger posterior, relative to anterior, hippocampal volumes in the right hemisphere tended to rate their use of map-based navigational strategies more highly” (Brunec, 2019, p. 750). More famously, "London taxi drivers have greater grey matter volume in the posterior hippocampus compared with control subjects, and for the right posterior hippocampus, grey matter volume varies positively with time taxi driving” (Maguire, 2003, p. 213). Perceiver thought thinks naturally in terms of maps and spatial connections. Thus, a long-term focus upon maps causes the right posterior hippocampus to enlarge.

One paper points out the relationship between the right hippocampus and Perceiver thought: "Several previous studies involving scene construction have noted the preferential engagement of the right hippocampus. We now extend this by showing that connectivity particularly with right anterior medial hippocampus was enhanced during scene construction. The right hippocampal involvement may be related to the visuospatial nature of the stimuli; such a preference for the right hippocampus has long been recognized” (Zeidman, 2014, p. 3850). Another paper specifically focuses upon the distinction between left and right hippocampus: "Our findings support the hypothesis that the left hippocampus plays a critical role in episodic verbal memory, while right hippocampus might be more important for spatial memory processing among non-demented older adults” (Ezzati, 2016, p. 2).

However, most papers do not distinguish between left and right hippocampi. This is probably because Contributor thought connects Perceiver facts with Server sequences, which means that the mind is seldom dealing with isolated Perceiver facts or Server sequences, but rather a combination of these two. Saying this another way, the two hippocampi usually deal with a combination of space and time rather than just space or time.

Perceiver thought comes up with facts by looking for connections between Mercy experiences. This makes it possible to organize Mercy experiences into different categories. The most common Perceiver categorization is that of objects within spatial locations. For instance, 'This is a rock. All the material in the rock is similar, and the rock is different than the ground below and the air above. The rock is sitting next to a tree.' Server thought performs a similar organizing and dividing along the dimensions of time, arranging similar actions into routines and recipes. For instance, 'This is the recipe for baking a cake. Those are the steps one takes to write an essay. When I am finished baking a cake, then I will write my 
essay.' Such organizing and dividing is happening within the hippocampus: "We propose that the hippocampus defines contexts as chunks of experience that have low variance across space and time" (Ekstrom, 2017, p. 8). A category changes when there is some kind of discontinuity: "Just as a large border (such as a wall) leads one to assume a boundary between two spatial contexts, large changes in the continuity of incoming information over time appear to trigger boundaries between events. Interestingly, both event boundaries and spatial boundaries modulate hippocampal activity” (p. 7).

Perceiver thought defines a context or category by looking for reference facts that do not change. Similarly, Ekstrom suggests that "we can expect the hippocampus to treat any cue that remains stable over a chunk of time spent in a given place as an indicator of the spatial context, and sufficient change in one of these cues can lead one to infer the existence of a new context” (p. 8). The hippocampus initially looks for categories within Perceiver space and Server time, but it typically goes beyond this to use other forms of categorizing: "The critical point is that the hippocampus uses regularities in the environment across time and space in order to define a context, and this initial representation must be established in order to learn what is behaviorally relevant in that context. Once the behaviorally relevant variables are determined, then these dimensions are added to the cognitive map” (p. 9). All facts and sequences are placed within some context: "Just as place cells remap when an animal is moved to a new spatial context, hippocampal time cells 'retime' when the temporal structure of a task, or the current behavioral context is changed. These findings demonstrate that hippocampal time and place cells 'map' experiences in relation to a context that is bounded in time and space. Consistent with the data from rodents, our labs have repeatedly shown that activity patterns in the human hippocampus carry information about the spatiotemporal context in which an object was encountered” (p. 7).

Summarizing, the hippocampus uses discontinuities to divide space and time into specific contexts, it uses fixed points within each context to define that context, and it then places more specific information within the appropriate context. This accurately describes how Perceiver thought functions. Presumably, it also describes how Server thought approaches sequences and actions. That is more difficult to ascertain, because we have discovered through personal experience that Server persons typically find it very difficult to describe how they think. Thus, one has to observe how the Server person behaves, and then come up with conclusions about cognition.

The hippocampus is a long strip that extends from anterior to posterior in the human and monkey. (The rat hippocampus extends from ventral to dorsal.) The posterior hippocampus handles details while the anterior hippocampus constructs the context within which these details are placed. "The posterior hippocampus, connected to perceptual and spatial representational systems in posterior neocortex, supports fine, perceptually rich, local details of memories; the anterior hippocampus, connected to conceptual systems in anterior neocortex, supports coarse, global representations that constitute the gist of a memory” (Sekeres, 2019, p. 39). A similar division can be seen when retrieving memories. "Initially locating a memory implicates the aPHC, whereas later elaboration of its details implicates the pHPC. Likewise, during navigation, the aHPC has been associated with initial global reinstatement of the environment and the pHPC with ongoing retrieval of local details” (Poppenk, 2013, p. 237). (aHPC is anterior hippocampus and pHPC is posterior hippocampus.)

The anterior hippocampus builds general concepts: "The work reviewed here implicates anterior hippocampus in concept formation. Not only has this region been shown to form integrated neural 
codes that capture commonalities across individual experiences, it has also been associated with uncertainty during concept learning. Relatedly, more complex memory functions that rely on integrating and organizing prior experiences such as autobiographical memory, schematic representation, and imagining the future have been distinctly associated with anterior hippocampus" (Mack, 2018, p. 7). A common form of general concept is the three-dimensional scene: "A circumscribed portion of the pre/parasubiculum in the anterior medial hippocampus was preferentially involved in scene construction. Importantly, our findings reveal for the first time that this region is preferentially recruited, not for mental construction per se, not for imagining a 3D space alone, but specifically for mental construction of scenes couched within a naturalistic 3D spatial framework (Dalton, 2018, p. 8156). (The subiculum is the primary output region of the hippocampus.)

General concepts in the anterior hippocampus build connections between many kinds of information. "Our finding accords well with the view and other reports that the anterior hippocampus is recruited for establishing links between conceptual information by combining multiple forms of information, such as semantic, spatial and emotional information or integrating distinct experiences on a conceptual or global scale” (Sheldon, 2016, p. 154).

The hippocampus does not just create general concepts. It also uses memories of general concepts to evaluate situations. If some new situation is similar to an existing general concept, then the situation will be regarded as familiar and the existing general concept will be used to evaluate the new situation. This leads to a sense of reasonableness, in which facts, sequences, and situations are evaluated based upon how closely they conform to existing general concepts. In contrast, if some situation is different than existing general concepts, then it will be regarded as novel. The Perceiver person evaluates new information by placing it within a mental context of reasonableness, and finds it disorienting and confusing when faced with situations that are truly novel. Similarly, the Server person finds it disorienting to be placed in a situation where one does not know what to do.

This process of organizing items into categories, looking for connections between categories, and evaluating new information based upon existing categories is described in the following quote: "Concepts define the relationships between similar objects; they represent combinations of features shared by objects of the same kind and allow us to recognize new instances of a concept when first encountered. Concepts also serve as the basis for inference about properties that have not or cannot be directly observed. To acquire a concept, we must experience multiple instances across unique episodes and learn both what features are common to concept exemplars and what features differentiate between concepts. Both of these operations, extracting commonalities across related experiences and distinctly representing similar experiences, are akin to episodic memory functions associated with the hippocampus. In particular, the hippocampus is thought to perform pattern separation to differentiate overlapping experiences into distinct memory representations. Pattern separation is complemented by memory integration, in which the hippocampus is thought to encode features of the current experience along with shared information from previously encoded experiences resulting in integrated memory representations that highlight commonalities across experiences” (Mack, 2018, pp. 1-2). Notice how confidence in a category increases with repetition. This is a fundamental aspect of Perceiver thought. Similarly, it is also possible to increase confidence in a Server sequence of actions through the repetition of practicing. 
Mentally placing a situation within a general concept leads naturally to what is known as boundary extension (BE). "BE is a ubiquitous cognitive phenomenon where we erroneously remember seeing more of a scene than was present in the sensory input, and occurs because when we view a scene, we implicitly and automatically extrapolate beyond the borders to form an extended internal representation of that scene” (Maguire, 2016, p. 433). For instance, if one sees a picture of part of a car, one will remember having seen the entire car. Boundary extension depends upon the hippocampus: "Patients with bilateral hippocampal damage and scene construction deficits had attenuated BE on all of these tasks... the initial BE effect, where the view is extended at the point of scene perception, is associated with increased hippocampal activity in healthy subjects” (p. 433). Boundary extension takes a partial experience and extends it mentally to fill the entire context: "One of the hallmarks of BE... is that BE is greatest for tight close-ups and decreases as more surrounding space is made visible (as in more wideangle views) until with very wide-angle views, no BE is observed” (p. 436). BE probably happens because the posterior hippocampus is adding detailed memories to the memory of a scene within anterior hippocampus: “[The] posterior hippocampus was engaged when healthy controls viewed simple scenes and experienced BE. Thus, posterior hippocampus may contribute predictions of what lies beyond the edges of the current view, to support the creation and updating of a scene model in anterior medial hippocampus” (Zeidman, 2014, p. 3846). A similar trait can be seen in the Perceiver person, who has a tendency to jump to conclusions based upon partial information, in essence filling in what is missing from some incomplete memory.

The anterior hippocampus can provide a factual framework for imagination. We have seen that the anterior hippocampus constructs mental scenes by pulling out common features from many similar situations. These mental scenes can be filled in with imaginary details instead of being populated by actual details from the posterior hippocampus. "When participants recalled episodic memories and imagined fictitious events set in the past or future (based on recombined elements from episodic memories) the amHipp was again the only part of the hippocampus significantly engaged for imagination and was part of a larger region activated during both imagination and recall whereas activation of posterior hippocampus was found specifically for vividly recalling real memories. Only amHipp was found to respond more strongly to imagining specific past or future events rather than general events” (Zeidman, 2016, p. 5). (amHipp refers to the medial bank of the anterior hippocampus.)

This describes the sort of imagination that I use to extend the theory of mental symmetry. Years of studying personality and analyzing systems have led within my mind to the development of many general concepts, and these general concepts now provide a framework for my imagination. Similarly, Richard Feynman, the famous physicist, said that "Science is imagination in a straitjacket” (Krauss). This may also explain the fascination that the typical Perceiver person has for science fiction, because science fiction creates mental scenes using facts and sequences associated with science, and then fills these scenes with imaginary people and events.

The anterior hippocampus connects with emotional regions of the brain including the amygdala. "Direct reciprocal links between the aHPC and the amygdala, insula, and vmPFC, as well as projections to the nucleus accumbens, offer the aHPC a privileged interface with motivational processing regions” (Poppenk, 2013, p. 232). “Turning to human evidence, dissection has revealed direct connections between the aHPC and amygdalar nuclei” (p. 231). (aHPC is anterior hippocampus.) 
This means that scenes or general contexts naturally acquire emotional overtones, because the amygdala is an emotional processor. This emotion can involve Mercy emotions of social interaction or personal experience: "Our main finding was that the autobiographical categories preferentially recruited the anterior hippocampus whereas the spatial categories preferentially recruited the posterior hippocampus” (Sheldon, 2016, p. 148). Notice that the non-emotional specific details are related to posterior hippocampus while subjective experiences involve the anterior hippocampus.

The anterior hippocampus can also become associated with Teacher emotions of order-withincomplexity. For instance, such order-within-complexity can be seen in autobiographical memory: "These results further support the emerging view that the anterior, as opposed to posterior, hippocampus integrates distinct experiences, thereby providing a scaffold for encoding and retrieval of autobiographical memories on the scale of our lives" (Nielson, 2015, p. 11078). Nielson adds that the left anterior hippocampus places biographical incidents within a more general framework of orderwithin-complexity: "The left anterior hippocampus represents space and time for a month of remembered events occurring over distances of up to $30 \mathrm{~km}$ ” (p. 11078). Nielson concludes "that the left anterior hippocampus represents space and time significantly better than the right anterior hippocampus” (p. 11080). This might suggest that the left hippocampus is associated with Perceiver thought and not with Server thought, but it could also mean that the left anterior hippocampus is being guided by the Teacher order-within-complexity of space-and-time.

Similarly, another paper examined hippocampal activity when moving through visual spaces portrayed on a computer. The researchers "found evidence of hierarchical encoding of this 3D spatial information, with the left anterior lateral hippocampus containing local corner information within a room, whereas RSC, parahippocampal cortex and posterior hippocampus contained information about the rooms within the building” (Kim, 2018, p. 1906). (RSC is retrosplenial neocortex, which is connected to the posterior hippocampus.) This might lead to the conclusion that the anterior hippocampus handles details, but a few paragraphs later, the paper mentions "that fMRI responses in the left anterior lateral hippocampus were associated with local corner information that was generalized across multiple rooms” (p. 1906). In other words, all the corners of the computer-generated rooms looked the same and this similarity was leading to a Teacher concept of order-within-complexity. As far as the hippocampus was concerned, a corner of a room was not a specific detail, but rather a general feature. However, because Teacher emotion is unknown, the authors conclude that "Our current results do not fit precisely with accounts that associate the posterior hippocampus with a finegrained spatial map. In fact, our findings could be interpreted as evidence in the opposite direction, namely that coarser-grained representations of the whole building engage the posterior hippocampus” (p. 1907).

Applying this to personality, it is possible to distinguish between Perceiver and Server persons who suppress emotions and those who acknowledge feelings. The Perceiver person can focus upon trivia, acquiring many specific, unemotional, disconnected facts that have no relationship to personal identity. This describes a mindset that emphasizes the posterior hippocampus. In contrast, the Perceiver person who acknowledges personal emotions also acquires the ability to use Perceiver thought to build connections between various contexts. Saying this another way, using the anterior hippocampus requires accepting personal Mercy emotions but it also makes it possible to be guided by Teacher emotions. The Perceiver person who uses the anterior hippocampus looks for 'the big picture'. Instead 
of merely collecting facts, the gist of a situation will be determined, and specific facts will be placed within the grid of some larger context.

A similar statement can be made about the Server person. The typical Server person focuses in a nonemotional manner upon performing specific actions in the present, presumably emphasizing the posterior hippocampus. Such a Server person becomes emotionally unstable when facing long-term uncertainties for which nothing can be done in the present. However, if the Server person can embrace this emotional instability, it then becomes possible to use Server thought at the higher cognitive level of being guided by an integrated Teacher understanding.

We have seen that the hippocampus looks for features that are common to similar situations. The hippocampus can also do the opposite, separating different situations which appear on the surface to be similar. One experiment measured what happens when people have to distinguish routes that partially overlap. "Here we found that hippocampal representations of overlapping spatial routes dramatically diverged with learning - to the point that overlapping routes were coded as less similar than nonoverlapping routes” (Chanales, 2017, p. 2312). The hippocampus mentally represented physically overlapping routes as different precisely where they overlapped and had to be mentally distinguished. “In contrast to orthogonalization, where overlapping memories are represented as 'unique,' a repulsion account holds that overlapping memories are represented as 'different from one another.' A repulsion account is not only consistent with the observed reversal effect but also readily explains the striking and seemingly paradoxical finding that the hippocampal reversal effect 'disappeared' precisely once routes diverged” (p. 2314).

A similar trait can be seen in the Perceiver person who is trying to distinguish truth from error. Attention will not usually focus upon the 'pagan' who has a totally different concept of truth. Instead, the Perceiver person will tend to attack the 'heretic' who believes many of the same things but differs in certain key areas.

Researchers are not certain exactly how to reconcile these apparently contradictory hippocampal traits of 'looking for what is common' and 'emphasizing what is different'. One author describes this contrast: "There is now a growing body of evidence for pattern separation signals in the hippocampus during encoding and work showing that variability in neural patterns across repeated testing is related to long-term retention. In the present experiment, hippocampal similarity across encoding was lower for overlapping trials relative to non-overlapping trials, consistent with this theoretical and empirical work. On the other hand, Cohen and Eichenbaum's relational memory theory hypothesizes that the hippocampus may support access to related memories through relational 'nodes' or neural ensembles that link overlapping memories” (Tompary, 2017, p. 236).

How the hippocampus responds may depend upon whether one is looking for differences or looking for similarities. Tompary explains that "the difference in the necessity or expectation of integration at encoding may dictate whether stimuli are integrated or separated” (p. 236). However, there will be a tendency towards integration if the categories are clearly defined, which implies that learning begins by defining the facts clearly before attempting to put these facts together. It was "found that neural patterns in a hippocampal cluster was biased toward integration only if the AB pairs were strongly learned in a separate block before participants were presented with BC pairs. Interestingly, in our study, 
encoding patterns in the hippocampus represented overlapping memories more distinctively than nonoverlapping memories, but then after a week of consolidation, retrieval patterns came to reflect integration” (p. 236). This implies that the Perceiver person who is 'attacking the heretic' is actually uncertain about his own beliefs and is attempting to clarify his own definition of truth.

Study of personality suggests that thought will become biased in the direction of the dominant emotion. If the mind contains a general theory within Teacher thought, then there will be a natural tendency to clarify Perceiver facts and Server sequences before placing them within some integrated understanding. In contrast, if the mind is guided by Mercy emotions of personal status, religion, or culture, then the primary focus will be upon distinguishing my person, my religion, and my culture from similar people, religions, or cultures.

Thomas Kuhn described the transformation in thinking that occurs when a group of people becomes guided by a paradigm in Teacher thought. "No period between remote antiquity and the end of the seventeenth century exhibited a single generally accepted view about the nature of light. Instead, there were a number of competing schools and subschools, most of them espouse in one variant or another of Epicurean, Aristotelian, or Platonic theory... being able to take no common body of belief for granted, each writer on physical optics felt forced to build his field anew from its foundations. In doing so, his choice of supporting observation experiment was relatively free, for there was no standard set of methods or a phenomenon that every optical writer felt forced to employ and explain. Under these circumstances, the dialogue of the resulting books was often directed as much to the members of other schools as it was to nature" (Kuhn, 1970, pp. 12-13).

\section{Medial Prefrontal Cortex}

We have looked so far at the four cognitive modules in isolation. The diagram of mental symmetry shows an arrow leading from Mercy to Perceiver and from Teacher to Server. Interaction between Perceiver and Mercy, and between Teacher and Server, occurs within the medial prefrontal cortex.

The medial prefrontal cortex is critical for maintaining a factual model of the current situation: "Understanding movies and stories requires maintaining a high-level situation model that abstracts away from perceptual details to describe the location, characters, actions, and causal relationships of the currently unfolding event. These models are built not only from information present in the current narrative, but also from prior knowledge about schematic event scripts, which describe typical event sequences encountered throughout a lifetime" (Baldassano, 2018, p. 9689). This paper explains that "Numerous recent studies, in both humans and animals, have explored how schemas are stored in the brain and how they influence ongoing processing. A critical region implicated in many of these studies is the medial prefrontal cortex (mPFC). This region shows encoding-related activity predictive of subsequent memory for schema-congruent stimuli, increased activity when remembering schematic knowledge, representations of temporal position within a schematic sequence, and upregulation of intermediate early gene expression when assimilating new information into a schema, with damage to the mPFC resulting in deficits for schema-related processing” (p. 9689).

Schemas were mentioned previously when looking at the frontopolar cortex, which I suggested is also related to Perceiver (and Server) thought. Thus, there appears to be a hierarchy from orbitofrontal to medial frontal to frontopolar. The orbitofrontal maintains an emotional model of the current context, 
which is related to Mercy thought (but may also include Teacher thought). The medial frontal constructs a schema that focuses upon the facts of the current situation, as represented by the arrow from Mercy to Perceiver. Finally, the frontopolar builds connections between various schema. Looking at this from the perspective of the Perceiver person, the Perceiver person feels as if he is pulling out factual information from Mercy experiences while being guided by a big picture that extends beyond the current context. Going further, a Perceiver fact can be defined as a connection that remains repeated over time. Baldassano explains that the "mPFC is one of the few regions that track past context over long enough timescales to support the long-term temporal dependencies encoded in full naturalistic scripts. (p. 9697). We saw earlier that Perceiver facts (and Server sequences) are determined by the dorsolateral frontal cortex. The medial frontal is then applying these facts to personal experiences.

The medial prefrontal cortex acquires the structure of schema from the hippocampus: “An entirely distinct line of work has implicated the mPFC in tasks that require or explicitly instruct the online integration of information with shared content. Activation of the mPFC and its connectivity with the hippocampus increases when encoding episodes containing stimuli that overlap with recently learned trials. Activation of mPFC is also related to retrieval-mediated integration and updating of existing memories both in humans and rodents. Furthermore, increased hippocampal mPFC connectivity has been observed during the retrieval of memories with regularities across episodes” (Tompary, 2017, p. $\underline{229})$.

Another paper examined what happens when a person has 'an insight' and realizes which facts fit together and which facts do not: "Insight triggers the emergence of de novo mnemonic representations of the narratives and is associated with increased neural similarity between linked event representations in the posterior hippocampus, mPFC, and autobiographical-memory network. Simultaneously, events irrelevant to the newly established memory of the narrative were pruned out. This process was accompanied by increased neural dissimilarity between non-linked event representations in the posterior hippocampus and $\mathrm{mPFC}$ and was additionally signaled by a mismatch response in the anterior hippocampus" (Milivojevic, 2015, p. 821). In other words, when there is some new insight, then the medial prefrontal guides the anterior hippocampus to come up with a new context, which then causes specific facts to become rearranged in the posterior hippocampus. This may be describing what happens in the brain during a paradigm shift.

The medial prefrontal also deals with self-image: “The medial prefrontal cortex (mPFC) has been implicated in seemingly disparate cognitive functions, such as understanding the minds of other people and processing information about the self. This functional overlap would be expected if humans use their own experiences to infer the mental states of others, a basic postulate of simulation theory" (Mitchell, 2006, p. 1306). As a Perceiver person, I naturally think of self-image as something related to the internal world of Perceiver and Mercy, but interaction between internal Teacher and Server thought would also generate a form of self-image-my personal Server skills in relation to universal Teacher understanding.

Self-image is related to conscience but is more personal. Perceiver thought looks for similarities between specific situations. This leads to an impersonal concept of the rule of law, in which one applies the same rules equally to everyone. We saw earlier that this sense of fairness involves the right dorsolateral frontal. Self-image, in contrast, looks for consistent facts about personal identity. Thus, 
both conscience and self-image involve an interaction between Perceiver facts and subjective Mercy experiences, but conscience adds personal identity to facts while self-image adds facts to personal identity. Self-image involves the medial prefrontal gyrus.

A distinction can between made between self and self-image. The emotional self is related to Mercy thought and orbitofrontal cortex, while self-image adds Perceiver facts to Mercy thought and is related to medial frontal cortex. The emotional self can be defined simply as the emotional models that cannot be ignored. In other words, Mercy thought can practice emotional identification and pretend for a while that anything is self. However, physical ability, skill, knowledge, and expertise will all force Mercy thought to return to a limited set of emotional models, which then become defined as self. Self-image can then be defined as the schema of self-the Perceiver facts (and Server sequences) that are common to all the emotional models that keep being reimposed upon orbitofrontal cortex. This distinction between self and self-image can be seen in the Mercy person and Perceiver person. The Mercy person focuses upon the experiences and emotions of self, whereas the Perceiver person focuses more heavily upon the facts of self-image.

One paper suggests that the medial frontal cortex can be subdivided into a ventral part that deals with self-image in a more emotional manner, and a dorsal part that deals with self-image and images of other selves in a more factual manner: "Although the human mPFC is neither uniquely nor solely devoted to social cognition, its central role in navigating the interpersonal space is probably one of the most often replicated findings in functional neuroimaging research... We comprehensively characterized both the vmPFC and dmPFC as relevant for self- and other- focused as well as social, emotional, and facial processing. More specifically, the vmPFC subserves predominantly nonambiguous subjective-value-related evaluative processes driven by bottom-up pathways, whereas the dmPFC subserves predominantly ambiguous amodal metacognitive processes driven by top-down pathways” (Bzdok, 2013, pp. 13-14).

A more recent paper summarizes an alternative interpretation, which is that the ventral part focuses upon self while the dorsal part thinks about others: "DMPFC/ BA 9 has been associated with social cognitive processes for both the self and others, whereas only MPFC/BA 10 preferentially supports self-reflection” (Meyer, 2018, p. 715). (The DMPFC/BA 9 is the dorsal medial prefrontal while the MPFC/BA 10 is the ventral medial prefrontal. BA stands for Brodmann area.) These two interpretations are quite similar because Perceiver and Server thought look for facts and sequences that are common to both self and others. This paper describes the functions of the ventral medial frontal in more detail: “MPFC/BA 10 has been associated with multiple aspects of self-reflection, including considering one’s own traits and feelings, as well as imagining oneself in the past or future. This region also engages when we consider other people who are highly self-relevant, such as individuals we are similar to, close with, and/or know a great deal about. In fact, recent findings suggest MPFC/BA 10 may be a social information hub with organized, self-relevant person and trait knowledge” (p. 719).

The purpose of the Meyer paper was to explore the connection between thinking about self and the 'default network': "MPFC/BA 10 is part of a brain system that generates disproportionate metabolic activity in the brain at rest. Neuroscientists have identified the 'default network,' so named because these brain regions reliably engage by default, whenever our mind is free from external demands” (p. 714). Going further, "Several theoretical papers and meta-analyses have discussed the overlap in 
MPFC/BA 10 during rest and self-referential processing, suggesting the concordance reflects that people engage in self-referential processing when they are not attending to the outside world" (pp. 714715). This relates to the suggestion made earlier that self can be defined as the emotional models that cannot be ignored. One can temporarily ignore self by focusing upon some task, object, or event, but attention will naturally return to self-hence a 'default network'. For instance, one sees this mental shift when driving a vehicle. A person who is driving does not typically think about occupying a vehicle but instead acts temporarily as if the vehicle is personal identity. Looking at this from a larger perspective, self is initially defined in a person by the limitations and abilities of the physical body, but over time this becomes expanded to include personal knowledge, skills, expertise, and character.

The relationship between the 'default network' and thinking about self can be seen in another paper that compared people who had recovered from depression with the average person. The authors summarize that "medial prefrontal cortex (MPFC) activity during self-referential processing has been associated with rumination and found aberrant in depression" (Nejad, 2019, p. 36) and they found that "proneness to ruminate tends to be positively correlated with the activity of the dorsal MPFC and dorsal ACC during self-referential processing in controls whereas the opposite pattern was observed in remitted patients” (p. 40).

As was mentioned previously, self-image can be defined either from a Perceiver perspective or from a Server perspective. The Perceiver-Mercy perspective views self as a sort of abstract object with certain features. But many of these features actually involve Server skills and Server actions: 'I can do these actions and I have done those actions.' Self can also be defined from a Server-Teacher perspective as a certain profession: 'I can perform all of the actions and procedures that are summarized by the general Teacher theory of some name, such as dentist, or plumber.' The Server perspective is usually more general than the Perceiver perspective. All professional dentists should be capable of carrying out similar skills. Therefore, one can go to see the dentist, and it does not really matter if one is dealing with Fred the dentist or Laura the dentist. In contrast, Perceiver thought works out the facts that distinguish self in Mercy thought from other individuals. These two perspectives can overlap. On the one hand, a professional can become more individual by specializing. On the other hand, an individual can become part of a culture that shares common skills and experiences.

The relation of the left medial prefrontal to professional skill can be seen in a study that looked at professional badminton players: "Compared to novices, badminton players exhibited stronger activation in the left medial frontal cortex during action anticipation and greater functional connectivity between left medial frontal cortex and some other brain regions (e.g., right posterior cingulate cortex). Therefore, the present study supports the position that the medial frontal cortex plays a role in more skilled action anticipation” ( $\underline{\mathrm{Xu}, 2016, \mathrm{p} .1})$. A similar distinction was found in professional tennis players: “The experts’ left medial frontal gyrus (MFG) cortex was significantly more active as compared to the right MFG when processing the overlap of tennis scene, but the lateralization effect was not significant in novices" (He, 2018, p. 1). And this probably relates to self image: "Experts have attentional superiority on skill-related scene information... This superiority is actuated by the activation of left MFG cortex and probably due to self-reference” (p. 1).

At this point, the concept of a mental network needs to be introduced. A mental network can be thought of as a schema with emotions. I came up with the idea of a mental network independently when 
developing mental symmetry but I realized the significance of a mental network when encountering the idea of the Agency Detection Device in the cognitive science of religion (Barrett, 2008). A mental network is a collection of emotional memories that functions as a unit. When some element of a mental network is triggered, then this will activate the entire mental network, which will then use emotional pressure to impose its structure upon the mind. A mental network will generate positive emotions if it experiences information that is consistent with its structure and negative emotions if it encounters information that is inconsistent with its structure. This emotion of consistency that comes from a mental network is distinct from the emotional labels that are attached to specific memories within a mental network. For instance, consider a person living in an abusive situation. The experiences themselves are emotionally painful, but the mental network that represents the situation will generate positive feelings of familiarity.

If a mental network continues to encounter inconsistent input, then it will start generating a hyper-pain that goes beyond normal negative emotions and it will begin to fragment. This hyper-pain will stop if a mental network is provided with input that is consistent with its structure. For instance, a person who leaves an abusive situation will feel an emotional drive to return to that kind of situation because it is familiar. In contrast, if the inconsistent information continues, then the mental network will eventually fall apart and revert to being a collection of isolated emotional memories. One can see this transition in the breaking of a habit. When mental networks come into contact with one another, then each will use emotional pressure to attempt to impose its content upon the other. This will lead to a hierarchy of mental networks. Study of personality indicates that human thought is ultimately based upon a set of core mental networks, and the mind will experience existential angst if core mental networks begin to fragment.

There are two kinds of mental networks: Mercy mental networks (MMN) and Teacher mental networks (TMN). An MMN will form whenever Mercy thought experiences a situation or set of similar situations with sufficient emotional intensity. A TMN will form whenever a sequence of actions is repeated enough times or a theory continues to be used. The mind uses MMNs to represent living beings, because a living being will be experienced as an object that generates many similar emotional experiences. Culture is also represented internally by MMNs. A habit is a TMN that results from repeating some action, while a paradigm is the TMN that results from continuing to use technical thought within some context. Self can be defined as the mental networks that are impossible to ignore. Self-image naturally emerges as Perceiver and Server thought learn which mental networks continue to reappear. My hypothesis is that the orbitofrontal region handles the emotional essence of mental networks, while the medial frontal determines the content of mental networks as well as connecting mental networks together. We will discuss how a mental network forms when looking at the basal ganglia.

The medial frontal cortex plays a primary role in Theory of Mind: "We meta-analyzed imaging studies on theory of mind and formed individual task groups based on stimuli and instructions. Overlap in brain activation between all task groups was found in the MPFC and in the bilateral posterior TPJ. This supports the idea of a core network for theory of mind that is activated whenever we are reasoning about mental states, irrespective of the task- and stimulus-formats” (churz, 2014, p. 9). (TPJ means temporo-parietal junction. We will not discuss the parietal or temporal lobes, but this region would 
presumably contain specific details involving Theory of Mind.) I have suggested that the medial frontal interconnects mental networks. Thus, Theory of Mind could be interpreted as using mental networks to predict how another person will behave. Encounters with friends and family members would trigger specific mental networks that represent these people, and these mental networks would then impose structure upon the mind that would predict how these people will behave. Strangers would trigger more generic mental networks, which would also attempt to predict a person's behavior, resulting in cultural, professional, or racial stereotypes. I suggest that stereotyping is impossible to avoid, because the mind cannot function without mental networks, but it is possible to make it more accurate and also gain the ability to rethink initial impressions.

\section{Anterior Cingulate Cortex}

The diagram of mental symmetry shows a connection between Perceiver and Contributor. Cognitively speaking, this means that Perceiver thought and Contributor thought work with the same information but interpret this information in a different way. (A similar statement could be made about Server and Contributor.) Perceiver thought looks for connections between facts, as demonstrated by the dorsolateral and frontopolar cortices. Contributor thought, in contrast, uses facts to guide plans.

Looking at this Contributor perspective, the anterior cingulate calculates cost and benefit. There are "times when animals decide whether it is worth acting at all or evaluate whether it is worth continuing to engage in the current behaviour or to explore alternatives. This distinct pattern of decision-making is linked to ACC [anterior cingulate cortex]; ACC manipulations affect the ability of animals to initiate any action at all, weigh up the costs and benefits of actions, switch between actions as their values change, or explore alternative choices” (Kolling, 2016, p. 37). Cost-benefit calculations are a fundamental aspect of Contributor thought.

We have looked at how Mercy and Perceiver interact, as well as how Teacher and Server interact, in the medial frontal cortex, and we have seen that this can lead to schemas and concepts of selves. These combinations lead at a more specific level to concepts of value. We will examine value first from a Perceiver perspective and then look briefly at the Server perspective. Perceiver thought organizes Mercy experiences into categories by looking for repeated connections, resulting in object detection. For instance, if one repeatedly notices wheels, engines, and metal bodies being combined together in a predictable manner, then one learns to recognize the object of a car. Value emerges when Perceiver thought organizes emotional Mercy experiences into categories. For instance, a car is not just an object, but it is also an object with an emotional label, and this emotional label determines the value of the car. Similarly, the parts of a car also have values, and the value of a car will change as the Mercy experiences that are connected with a car or its parts acquire emotions. For instance, a car may acquire sentimental value because it was used on a memorable trip, or it may lose in value because the engine needs a major repair. Neurologically speaking, the transition from emotional label to value happens when information is sent from the orbitofrontal cortex to the anterior cingulate: “Orbitofrontal cortex neurons focus on reward value representations for stimuli and know little about actions. The orbitofrontal cortex sends inputs to the ACC about the value of stimuli, that is, about goals including the value of outcomes (the reward received) and the expected value” (Rolls, 2019a, p. 4). 
Going further, I have mentioned that Contributor combines Server and Perceiver. When Server actions become added to Perceiver objects, then this leads to a concept of cause-and-effect. For instance, a car contains various components that are spatially connected. Cause-and-effect, in contrast, contains various components that are temporally connected by Server sequences. For instance, dropping an object is followed by the object hitting the ground. Cause-and-effect will be viewed by Perceiver thought as an object that involves time, and it will be viewed by Server thought as a sequence that occurs within a certain context.

Cost-benefit emerges when Server actions become added to Perceiver concepts of value. For instance, the vase that I am holding in my hand may have great value, while the fragments of a vase that lie on the ground after it has been dropped have much less value. In addition, the Server sequence that leads from cause to effect also has an emotional label; moving from cause to effect often involves significant emotional discomfort. Using neurological language, “The anterior cingulate cortex is implicated in emotion, because it is involved in linking reward and punishment information, which elicit emotional responses, to behaviour, and, in particular, to actions” (Rolls, 2019a, p. 2).

Teacher emotion also plays a role in both value and cost-benefit. For instance, a car contains many components that work together in harmony, an example of order-within-complexity. An engine that needs a major repair is an example of chaos-an exception to the general rule, a lack of order-withincomplexity. Similarly, a vase in my hand has much greater order-within-complexity than the fragments of a vase lying on the ground.

Turning now to the perspective of Server thought, Server actions that are carried out in a smooth and integrated manner lead to Teacher feelings of elegance. Contributor thought connects Server actions with Perceiver objects, leading to the concept of machines. A machine is combined of parts, and each part has a function: it is a Perceiver object that carries out some Server sequence. A functioning machine, such as a working car engine, creates Teacher feelings of order-within-complexity, because many parts are working together in an integrated manner. Notice that the same machine can be viewed by Mercy thought as a valuable object or by Teacher thought as an example of order-within-complexity. The first describes the typical viewpoint of the consumer, while the second describes the viewpoint of the mechanic.

Cost-benefit also emerges with machines. Machines are composed of parts, and each part has a value. Assembling a machine takes effort, and this effort has an emotional cost. For instance, if an older car has a major engine problem, then it may be cheaper to replace the car than fix the engine. Cost-benefit can be influenced by Teacher emotions. For instance, a sequence of actions that is smooth and elegant tends to cost less than a sequence of actions that is complicated and convoluted.

Summarizing, Rolls proposes "the framework that the value representations computed in the orbitofrontal cortex where there is little representation of action are transferred to the anterior cingulate cortex, where they can be used as the representation of reward vs non-reward or punishment outcome to be associated with representations of actions as part of goal-dependent action-outcome learning” (Rolls, 2019a, p. 8). "In the same dorsal anterior cingulate area, neurons were more likely to take into account the costs of the actions needed to obtain rewards, as well as the probability of obtaining the 
reward, than were orbitofrontal cortex neurons” (p. 8). Notice how the anterior cingulate is adding a sense of time and sequence to static feelings of reward within the orbitofrontal cortex.

Another researcher describes it this way: “Activity in anterior cingulate cortex (ACC) has been linked both to commitment to a course of action, even when it is associated with costs, and to exploring or searching for alternative courses of action. Here we review evidence that this is due to the presence of multiple signals in ACC reflecting the updating of beliefs and internal models of the environment and encoding aspects of choice value, including the average value of choices afforded by the environment" (Kolling, 2016, p. 36). Saying this more succinctly, “ACC manipulations affect the ability of animals to initiate any action at all, weigh up the costs and benefits of actions, switch between actions as their values change, or explore alternative choices” (p. 36). One sees here a combination of self-image and cost-benefit. Cost-benefit is not just being calculated in some objective manner. Instead, it is being used to determine self-image: 'This is an opportunity' is being turned into 'I will commit to this opportunity; this is my plan and it will define my self'.

This overlap between planning and self-image appears to center in the perigenual anterior cingulate (pgACC). (There is the perigenual anterior cingulate and the pregenual anterior cingulate. These overlap, but I am not certain how they precisely relate because papers use either one term or the other but not both.) The "PgACC, partly through its interactions with ventral striatum, is important for motivating effortful sequential search decisions over time. In contrast to dACC, which carried strong signals related to the planning of an extended behavioral sequence, pgACC activity was more closely related to the motivation to engage in the sequence" (Kolling, 2018, p. 1080). (Notice in passing the connection with the ventral striatum, which will be discussed later.) Similarly, a human study found that "Positive coping styles were associated with increased ACC volume, specifically with the perigenual extending to the subgenual part” ( to plans that can improve my personal situation.

Another study looked at patients with frontotemporal dementia and found that "right pregenual anterior cingulate cortex (pACC) gray matter volume was the only brain region that was a significant predictor of self-conscious emotion” (Sturm, 2013, p. 468). The self-conscious emotion in this study was triggered by playing back a video of the patient singing karaoke. Based upon personal experience, I suggest a possible explanation. I am a professionally trained violinist. I have found that experiences of musical inadequacy can trigger two kinds of emotional response within my mind. The normal emotional response is that of professional evaluation, presumably guided by feelings of professionalism within the left medial frontal. But there are times when a performance is so cringe-worthy that I start to feel personally embarrassed, leading to a desire to extricate myself personally from the situation. I suspect that this feeling of personal embarrassment involves the right pregenual anterior cingulate.

A recent study based in brain stimulation of epileptic patients confirms this distinction: "We found clear functional differences between the pregenual part of the cingulate cortex, hosting the majority of emotional, interoceptive and autonomic responses, and the anterior midcingulate sector, controlling the majority of all complex motor behaviours" (Caruana, 2018, p. 1). The complex motor behaviors included "getting-up impulses, reaching and grasping actions, body-directed actions, and exploratory eyes-head movements. All these behaviours were constituted by the combination of simple movements into an integrated pattern, and they were often executed in a smooth natural way” (p. 5). This describes 
fragments of various plans of action. In contrast, "The result of electrical stimulation of the pregenual sector of ACC assigns to this region an unequivocal role in determining motor behaviours linked to affective functions, the most common response consisting in the production of emotional facial displays, interoceptive sensations or autonomic responses” (p. 13). This describes various components of how one might respond to the 'cringe-worthy' performance mentioned in the previous paragraph.

The pregenual anterior cingulate also makes value more personal by conveying sensations of physical pain and pleasure: "Pain activates an area typically 10-30 mm posterior to and above the most anterior (i.e. pregenual) part of the ACC. Pleasant touch activated the pregenual cingulate cortex. Pleasant temperature applied to the hand also produces a linear activation proportional to its subjective pleasantness in the pregenual cingulate cortex. Somatosensory oral stimuli including viscosity and the pleasantness of the texture of fat in the mouth also activate the pregenual cingulate cortex” (Rolls, 2019a, p.7). These personal sensations can be modified by triggering various mental networks: "Pleasant (sweet) taste also activates the pregenual cingulate cortex where attention to pleasantness and cognition also enhances activations. Pleasant odours also activate the pregenual cingulate cortex, and these activations are modulated by word-level top-down cognitive inputs that influence the pleasantness of odours, and also by top-down inputs that produce selective attention to odour pleasantness" (p. 7). And the pregenual cingulate cortex can also be activated by the bottom line of money: "Moreover, the outcome value and the expected value of monetary reward activate the pregenual cingulate cortex” (p. 7).

When the Contributor person carries out a plan, then Contributor thought will impose some sort of mental grid upon imagination. (All cognitive styles can do this to some extent, but the Contributor person appears to have a natural talent for this ability.) For instance, a professional golf player who is imagining a shot will internally see both the path of the ball and a mental grid within which the ball travels. Similarly, in the diagram of mental symmetry the arrow that leads from Exhorter to Contributor represents the internal visualization of some predicted path, while the lines that connect Contributor with Perceiver and Server represent the mental grid within which this predicted path is placed.

This mental grid appears to be coming from the posterior cingulate cortex: "The posterior cingulate cortex receives information from the nearby neocortical areas such as the parietal cortex about spatial representations, and projects this onwards via the parahippocampal cortex to the hippocampus (which is allocortex), where it provides the spatial component for object-space episodic memories. This provides for a memory-related function of the cingulate cortex... The anterior cingulate cortex receives information from its topologically nearby neocortical area, the orbitofrontal cortex” (Rolls, 2019a, p. 13). Putting this all together, "The ACC [anterior cingulate cortex] receives reward and punishment outcome information from the orbitofrontal cortex (OFC). The posterior cingulate cortex receives information about actions from the parietal cortex. Then these two types of information are brought together towards the mid-part of the cingulate cortex including the cingulate premotor area, which with its connections to premotor neocortical areas can select the action that is most likely, given the actionoutcome learning performed within this cingulate system, to obtain the goal, the desired outcome” (p. 11). Saying this cognitively, Contributor thought assembles a plan within the mid-cingulate by placing value and projected outcome from the anterior cingulate within a mental grid from the posterior cingulate. (This mental grid is calculated by the hippocampal region.) 
Summarizing, this section has looked at the relationship between the anterior cingulate and concrete goal-oriented behavior, and we have seen that the dorsal anterior cingulate cortex evaluates costs and benefits from a more objective perspective while the perigenual anterior cingulate takes a more personal perspective. We will look further at this distinction when discussing the basal ganglia and the role played by the striosomes.

Mental symmetry suggests that the anterior cingulate also plays a role in abstract thought, guided primary by the Teacher goal of achieving order-within-complexity, typically by improving some theory or solving some problem. Evidence for this is more difficult to find within neurology. I suggest two possible reasons: First, most neurological understanding is based in animal studies. This is useful, but it will not lead to an understanding of abstract thought, because animals are largely incapable of abstract thought. Second, we saw earlier that neurology does not recognize Teacher emotion, and Teacher emotion provides the primary motivation for abstract thought.

\section{Amygdala}

The amygdala has traditionally been associated with fear, and one still occasionally finds papers describing fear as the primary function of the amygdala. However, it has become clear that the amygdala is an emotional processor that adds emotional labels to situations. "It has long been known that the amygdala, a bilateral structure from the medial temporal lobe, is related to emotion, particularly in processing of aversive information. However, accumulating evidence suggests that amygdala activation is also involved in processing pleasant information, as observed, for instance, in studies using reward-learning, episodic memory encoding, pleasant scene or face perception or mental imagery of pleasant experiences” (Weymar, 2016, p. 1).

Similarly, a meta-analysis paper that examined 148 imaging studies on the amygdala concluded that "Our results confirm that the amygdala responds to both positive and negative stimuli, with a preference for faces depicting emotional expressions” (Sergerie, 2008, p. 811).

I suggested previously that the two hippocampi are the processors for Server and Perceiver thought. Similarly, I suggest that the two amygdalae are the processors for Teacher and Mercy thought. They calculate an emotional label for the current situation, with Teacher thought using the left amygdala and Mercy thought the right amygdala. We saw when looking at the hippocampus that there is strong evidence connecting Perceiver and Server thought with the two hippocampi, but only partial evidence for associating the left hippocampus with Server thought and the right hippocampus with Perceiver thought. However, at least the two dimensions are well defined: Server thought emphasizes time and sequences while Perceiver thought emphasizes space and objects. And it makes sense that there is significant overlap between these two because Contributor thought builds connections between Perceiver facts and Server sequences.

Similarly, the emotion that one feels is the sum of Teacher and Mercy emotion, and as far as I can tell, these two kinds of emotion feel the same. (Teacher emotion may be felt more in the head, and Mercy emotion more in the gut, but that is merely a tentative suggestion.) Finding evidence of laterality in the amygdalae is somewhat difficult because neurology is not aware of the concept of Teacher emotion. But there is some indirect evidence. For instance, the meta-analysis by Sergerie "compared the number of activations in left and right amygdala for block and event-related designs separately. Whereas no 
difference between hemispheres in the number of activations was observed for event-related studies ( $p>0.5$ ), significantly more activations were reported in the left than in the right amygdala for experiments using a block design ( $p=0.007)$ ” (p. 819). If one examines what is meant by 'block and event-related', one notices that this is describing the presence or absence of Teacher order-withincomplexity. On the one hand, "block designs typically involve the repeated presentation of stimuli of the same category (e.g., positive, negative or neutral) for relatively long periods of time (the mean block duration for studies included in this meta-analysis was 107 s)” (p. 822). Presenting a series of similar stimuli will lead to Teacher feelings of order-within-complexity. On the other hand, "the pseudorandom stimulus presentation order used in event-related designs would prevent such a habituation from taking place” (p. 822). Pseudo-random events lack Teacher order-within-complexity. Thus, one concludes that the left amygdala is responding preferably to Teacher order-withincomplexity. Sergerie concluded that "This finding supports the often observed hemispheric differences in temporal dynamics and/or habituation rates, namely a short-duration response in the right amygdala and a more sustained one in the left” (p. 822). This may be an accurate distinction, because orderwithin-complexity only emerges over time. Looking at this from a cognitive perspective, Mercy thought tends to focus on specific experiences while Teacher thought looks for general theories.

Going further, one study found that "Partaking in meditation and yoga practices was associated with a significantly lower right amygdala volume” (Gotink, 2018, p. 1631). More specifically, they reported that the right amygdala was smallest in the meditation group as opposed to the breathing exercise participants and the yoga group. I mentioned previously that meditation (and mysticism in general) can be explained cognitively as using overgeneralization to come up with a universal Teacher theory of cosmic unity. Overgeneralization generates a theory by ignoring specific experiences rather than building upon them. This would cause the right amygdala to be underemphasized.

Looking at this from the other side, "The left amygdala GMV was larger in patients with later onset and smaller in cases of prolonged depression. In line with prior reports of depressed patients responding to antidepressant treatment, amygdala GMV was negatively related to illness duration, suggesting volume loss with disease progression” (Zavorotnyy, 2018, p. 229). (GMV is gray matter volume.) This may appear at first glance to contradict the suggestion made earlier that depression can result from coming up with a general Teacher theory of personal misery. However, a distinction needs to be made between a general theory and the emotions of the moment. In depression, a general theory of misery in the ventromedial frontal cortex is presumably driving the mind to focus upon the emotions of unpleasant Mercy memories being generated by the right amygdala. This theory of personal misery is being reinforced by mental networks from the part of the anterior cingulate that relates to physical identity: "Findings indicated a relationship between rumination and functional connectivity of the amygdala and subgenual cingulate that was greater in MDD-ever children than healthy children during emotion regulation (cognitive reappraisal)” (Murphy, 2016, p. 96). (MDD-ever refers to children who have had major depression at some previous time, and subgenual cingulate is next to perigenual cingulate.)

Teacher emotion can also be seen in humor: "A dominant theory of humor comprehension suggests that people understand humor by first perceiving some incongruity in an expression and then resolving it. This is called 'the incongruity-resolution theory'... incongruity resolution evoked positive emotion and activated the left amygdala” (Nakamura, 2017, p. 553). In other words, the left amygdala generates 
positive Teacher emotion when verbal confusion is suddenly followed by comprehension, because order is being brought to complexity.

Laterality can also be seen in math anxiety, which is a negative emotional response to mathematics. "High math anxious children showed increased activity in the right amygdala while performing mathematical computations. The present work extends this finding beyond the scope of children doing arithmetic math computations, as these results illustrate that even the mere presentation of mathematical expressions, without ever asking these participants to solve the expressions, is sufficient to cause increased amygdala reactivity in HMA young adults” (Pizzie, 2017, p. 17). “As math anxiety increases, activity in the right amygdala increases during trials where subjects are viewing mathematical expressions” (p. 12). We saw earlier that math generates Teacher emotions of beauty, but a person has to learn to appreciate the beauty of mathematics. A person with math anxiety lacks this Teacher understanding and will approach mathematics from a concrete Mercy perspective as a source of painful, personal experiences.

Moving on, I mentioned previously that the Mercy person is sensitive to a person's eyes. Consistent with this, "the amygdala, at least in humans, appears to be remarkably specialized for processing a single feature within faces: the region around the eyes. For instance, lesions of the amygdala selectively impair processing information from the eye region in order to judge facial emotion, and BOLD-fMRI studies reveal amygdala activation during attention to the eyes in faces and to isolated presentation of the eye region” (Rutishauser, 2011, pp. 1658-1659). Looking at this in more detail, "The left amygdala activated only to fearful eyes. Contrasts confirmed that the left amygdala response to fear was significantly greater than the responses to any other condition. Somewhat unexpectedly, the right amygdala responded to all conditions. Contrasts between conditions indicated that there were no significant differences between fear and other conditions in the right amygdala” (Hardee, 2008, p. 50). In other words, the right amygdala has a general sensitivity to the eyes, whereas the left amygdala is only activated when fear is noted in the eyes. Looking at this cognitively, the mind represents people as mental networks within Mercy thought. A person's eyes can help Mercy thought to determine which mental networks are active within the mind. Saying this another way, the eyes provide useful clues for Theory of Mind.

Turning to the left amygdala, fear implies the possibility of personal harm. A person is not just being faced with an unpleasant experience. Instead, the integrity of the mind or body is being threatened; the order-within-complexity of the mind or body may fragment into chaos and complexity. This type of functional threat will involve Teacher emotion and presumably the left amygdala.

Eyes are psychologically related to what is known as the 'uncanny valley': “The unease induced by altering eyes may help to explain the uncanny valley, which is the eeriness of robots that are almostbut not quite-human” (Schein, 2015, p. 173). This paper suggests that Theory of Mind can be divided into two emotional components, one related to Teacher feelings of order-within-complexity, and the other to Mercy feelings of personal experience: "Research on mind perception reveals that we perceive minds along two broad dimensions of agency (intending, planning, doing) and experience (feeling, sensing, consciousness), and that we have fundamental expectations about who or what should have a mind. Adult humans are generally expected to have both agency and experience, children and animals are expected to have only experience, and robots are expected to have only agency. As they are 
unliving creations of metal, robots are fundamentally expected to lack abilities that are central to living creatures of flesh - the capacity to feel. Therefore, mind perception suggests that uncanniness arises not from humanlike appearances per se, but when these appearances lead people to ascribe experience to robots. In other words, robots are creepy when they seem to feel” (pp. 173-174). Using cognitive language, a machine-like a robot—is an example of Teacher order-within-complexity, because many parts are functioning together in an integrated manner. The mind uses MMNs (Mercy mental networks) to represent living beings, but an adult human also becomes characterized by some profession or area of expertise-which is an example of Teacher order-within-complexity. Uncanniness is the feeling that arises when a machine triggers MMNs that are used to represent living creatures, and this is related to the presence or absence of eyes: "Without eyes, humans are perceived to lack experience, violating a fundamental expectation of mind and inducing uncanniness” (p. 177).

Looking at this from another context, autistic individuals find it difficult to look in people's eyes: "Individuals with autism spectrum disorder (ASD) often report that looking in the eyes of others is uncomfortable for them, that it is terribly stressful, or even that 'it burns'. Although traditional theoretical accounts of ASD have interpreted lack of eye contact and other social difficulties as indicators of interpersonal indifference to others, first hand reports from verbal people with ASD would rather suggest that the underlying problem may be one of socio-affective oversensitivity" (Hadjikhani, 2017, p. 1). In contrast, autistic individuals typically find emotional comfort in order and structure. This implies that autistic individuals prefer TMNs to MMNs. A TMN can be constructed out of simple components; one simply needs to interact with some system of order-within-complexity long enough for a Teacher mental network to form. In contrast, MMNs form intuitively as one interacts with living beings.

This leads to a strange conclusion. TMNs are inherently simpler than MMNs, but the mind has to be taught to consciously recognize Teacher emotion while we will see in the next section that even the childish mind can recognize Mercy emotion.

\section{Cognitive Development}

Jean Piaget did groundbreaking work on cognitive development in the mid-20th century. The general shape of his stages of cognitive development can be seen in neurology and explained using the theory of mental symmetry.

Mental symmetry suggests that the mind of the child is initially based upon MMNs within Mercy thought that the child acquires from physical experiences of pain and pleasure. This describes Piaget's sensorimotor stage. This emotional programming of the childish mind is driven by the amygdala: "The amygdala exhibits early functionality. Specifically, studies have revealed a robust functional responsiveness to emotional stimuli by early childhood, which is larger in magnitude than the responses of older individuals. Consistent with these findings, a rich animal and human literature examining the effect of both lesions and stress on the amygdala across the lifespan suggest that this region's role in shaping emotion and social behavior is especially important during early postnatal development, with consequences for affective behavior lasting throughout life. These findings suggest a sensitive period for human amygdala development in the late infancy to childhood period when the amygdala is particularly receptive to environmental stimulation” (Tottenham, 2017a, p. 2). 
Mental networks that represent parents and other authority figures also play a prominent role in the mind of the child. That is because a child has many emotional experiences of relying upon parents for care and protection, and these emotional experiences will lead to the formation of potent MMNs within the mind of the child. Consistent with this, there is evidence that emotional responses from the mother activate mental networks stored within the orbitofrontal cortex of the child: "It seems that the mother's smiling evokes the neural network involved in reward processing, especially the mesolimbic reward circuit, the front end of which is the OFC” (Takamura, 2016, p. 49).

Putting these two factors together, the response of the amygdala in a young child is strongly influenced by the emotional response of parents: "A dysregulated parent (exhibiting fear) is highly effective in increasing amygdala engagement and aversive learning about environmental stimuli. Evidence from humans is strikingly consistent with the rodent work. As in the developing rodent, it has been shown that parental presence buffers against children's elevations in stress hormones, parental stimuli attenuate amygdala activity in children, and fear expressed by the parent directly translates into fear related behaviors in the child. Thus, the parent is a highly effective regulator during development, with the ability to potentiate or attenuate amygdala function” (Tottenham, 2017a, p. 3).

This parental control over amygdala response in the child occurs primarily with the right amygdala, which I suggest is related to Mercy thought. This parental control would be dominant during Piaget's preoperational stage, because it fades in adolescence: "Specifically, right amygdala activity (which is typically high in childhood) was suppressed when children were viewing pictures of their mother, relative to when viewing pictures of a stranger. Critically, there was no difference in right amygdala reactivity in adolescents viewing pictures of mothers and strangers” (Callaghan, 2016, p. 168). Notice that right amygdala activity is 'typically high in childhood', consistent with the idea that Mercy thought is dominant in the childish mind.

During adolescence, the emotional balance shifts and the amygdala becomes controlled by a network of mental networks within the medial prefrontal cortex: "In adulthood, the medial PFC sends projections to inhibitory cells within the amygdala that reduce amygdala reactivity and are thus fundamental to mature affect regulation. Unlike in the adult, these regulatory influences from the medial PFC are not available to the young child. Several studies have now shown that functional connections between the amygdala and the medial PFC are immature in childhood and switch to the adult-like state in adolescence. That is, during childhood the amygdala is less likely to be regulated by the mPFC than after childhood. Instead, during infancy and childhood, external agents (e.g., caregivers) can serve as social regulators of affect and amygdala activity during this time” (Tottenham, 2017a, pp. 3-4).

Because the amygdala within the mind of a child is not regulated by the medial prefrontal cortex, the childish mind is emotionally volatile and will respond emotionally when separated from parents: "The strong early reactivity in the amygdala parallels the normatively high fear and emotionality that characterizes childhood; indeed, early childhood fears (ie, separation anxiety) are mediated by the excessively high amygdala activity observed during childhood” (Callaghan, 2016, p. 167).

Looking at this more generally, emotional outbursts of anger occur when the amygdala overwhelms the medial prefrontal cortex. There is "an early increase of bilateral amygdala activation during anger induction, which was then followed by increased left VMPFC activation during the anger imagery 
period... The amygdala's early activation suggests that the amygdala might be more involved in the initial experience of anger while the VMPFC is more involved in the evaluation of that anger and in the inhibition of an impulsive response" (Dougherty, 2017, p. 6). (VMPFC is ventral medial prefrontal cortex. This is a slightly imprecise term which includes most of the orbitofrontal as well as the medial prefrontal. This area would include mental networks as well as connections between mental networks.) Similarly, a study of patients with brain lesions "found that vmPFC lesions were associated with increased right amygdala reactivity to aversive stimuli” (Motzkin, 2015, p. 8). In other words, a lack of regulation from the vmFPC allowed the right amygdala to respond freely to emotional situations.

Separation from parents will cause the childish mind to shift from the medial prefrontal being programmed by the amygdala to the medial prefrontal regulating the amygdala. Callaghan notes that "Animal studies appear to converge on the finding that the developmental change from plastic to stable amygdala-mPFC circuitry is accelerated following chronic parental deprivation. Put another way, the data support the idea that early independence from the parent cues early termination of the sensitive period for environmental input into amygdala-mPFC circuitry” (Callaghan, 2016, p. 170). Looking at this cognitively, a mental network will fragment if it continues to experience input that is inconsistent with its structure. Such fragmentation will happen to childish mental networks that represent parents if parents are not physically present. When core mental networks within the mind fragment, then the mind has to find integration in other mental networks. Thus, mental networks that represent parents will become replaced by a network of mental networks within medial frontal that include parents, authority figures, and social structures.

This transition from childhood dependence to adolescent semi-independence is least painful if the developing child regularly alternates between parents being present and absent: "Experiencing a balance with autonomy is an additional component for healthy development. We have hypothesized that the regular daily separations and reunions that occur between sensitive parents and children (e.g., parents can go to work, and children can go to school) may benefit emotional circuitry" (Tottenham, 2017b, p. 101).

We have looked so far at the interaction between the amygdala and the medial prefrontal. The amygdala is dominant in the mind of the child, and emotional information from the amygdala programs the medial frontal. In contrast, the amygdala in the adult mind becomes regulated by a collection of mental networks coordinated by the medial frontal. This describes the development of Mercy thought within the mind of the child. The adult mind can go one step further and use Teacher thought to reprogram Mercy emotions. This is known psychologically as cognitive reappraisal. Using the language of Piaget, cognitive reappraisal becomes possible when cognitive development reaches the formal operational stage.

Cognitive reappraisal looks at an emotional situation from a different perspective, and it is widely used in psychology because it is effective: "The best-studied strategy is cognitive reappraisal, which targets the appraisal stage and involves changing one's interpretations or appraisals of affective stimuli. One reason this strategy is so well studied is because reappraisal is highly effective at regulating affect and physiological arousal without the cognitive and physiological costs associated with response-focused strategies (e.g., expressive suppression), and with longer-lasting effects than attention-focused strategies (e.g., distraction). But it is also well studied because the core elements of reappraisal are 
central to many forms of therapy, including cognitive behavioral therapy, dialectical behavioral therapy, and psychodynamic therapy, all of which are effective for treating a variety of mood and anxiety disorders" (Buhle, 2013, p. 2981).

Cognitive reappraisal will change the response of the amygdala: "We found strong evidence that reappraisal modulates activity in bilateral amygdala, but not other regions related to emotional responding” (p. 2986). However, this change is not being caused primarily by mental networks in the vmPFC. Buhle's meta-analysis "found that the implementation of reappraisal in the current set of studies did not consistently recruit vmPFC" (p. 2984). This suggests that reappraisal goes beyond the interaction between amygdala and ventromedial prefrontal that we have just discussed. Instead, "the implementation of reappraisal consistently activated domain-general cognitive control regions, including dmPFC, dlPFC, vlPFC, and posterior parietal lobe” (p. 2987).

Looking at this more carefully, "Cognitive reappraisal refers to deliberately viewing an emotionally evocative event from a different perspective and re-interpreting its meaning, thereby changing its emotional impact” (Papousek, 2017, p. 577). The left inferior frontal (otherwise known as ventrolateral prefrontal) plays a major role in reappraisal: "During cognitive reappraisal efforts, individuals higher on the capacity for generating cognitive reappraisals showed more left-lateralized activity in lateral prefrontal cortex, specifically in ventrolateral prefrontal cortex extending toward the frontal pole" (p. 577). In other words, cognitive reappraisal does not change the situation in Mercy thought. Instead, it changes the Teacher theory that one uses to explain the situation —it is a cognitive reappraisal. It looks at the same situation through a different set of theoretical glasses. Saying this another way, cognitive reappraisal is like a paradigm shift, because a person is using a new theory to explain the same set of facts.

Teacher thought constructs general theories by looking for simple explanations that can summarize many related situations. This search for order-within-complexity is carried out by the left inferior frontal, and a discovery of order-within-complexity will lead to positive Teacher emotion. Language itself is an example of Teacher order-within-complexity because a word is a simple sequence of phonemes that can be used to describe many similar items. Thus, language brings order-withincomplexity to the complexity of human experiences, and language itself can then be used to achieve further order-within-complexity through the verbal formulation of general theories. A young child can use Teacher thought at the level of phonemes and language, but it is within adolescence that the mind becomes capable of using Teacher thought at the higher level of words and general theories.

We looked earlier at math anxiety. It can be treated by a form of cognitive reappraisal: "Eight weeks of one-to-one tutoring reduced math anxiety in HMA [high math anxiety] children and tutoring-induced reductions in right amygdala activity predicted individual reductions in math anxiety." (Supekar, 2015, p. 12580). Going further, math anxiety involves excessive interaction between left and right amygdala: "Differences in the effective connectivity of the amygdala during arithmetic problem solving between HMA and LMA groups that were evident before tutoring were entirely absent after tutoring. Before tutoring, during mathematical problem solving, children in the HMA group, compared with the LMA group, showed greater effective connectivity between the right and the left amygdala” (pp. 1258012581). The tutoring is providing Teacher thought with positive emotions of order-within-complexity, and this is causing Mercy thought to feel differently about encountering experiences of math. 
A rational general theory uses Teacher thought to assemble the mental 'bricks' of Perceiver facts and Server sequences into the 'building' of a general theory. This is different than overgeneralization, which creates the feeling of a general theory by ignoring facts and sequences. The constructing of a general theory typically goes through the three stages of gathering Perceiver facts and Server sequences, coming up with a general Teacher theory that explains these facts and sequences, and then imposing this general theory upon facts and sequences. A similar sequence can be seen in the scientific method, where one gathers data, forms a hypothesis, and then makes a prediction.

These three steps can be seen in cognitive reappraisal. First, the dorsolateral prefrontal gathers data for Teacher thought: "Our results suggest that during the emotion regulation process, first the DLPFC together with the SMG might be involved in directing attention to the emotional stimulus, and the DLPFC might then play a role in actively maintaining the content and the goals of one's reappraisal in working memory... represented in positive connectivity between the DLPFC and the IFG” (Morawetz, 2016a, pp. 1933-1934). (This paper examined the left DLPFC and the left IFG, which represent Server and Teacher thought. SMG is supramarginal gyrus, which is between parietal and temporal lobes.) Second, Teacher thought comes up with a hypothesis: "When multiple representations of stimulusappropriate reinterpretations are activated, a selection is needed to resolve competition among the various representations to drive goal-directed behavior. This selection process has been associated with IFG activity” (p. 1934). Third, Teacher thought asserts this hypothesis as a general theory: "The final selection of a stimulus-appropriate reappraisal could then trigger the inhibition of the DLPFC because this region is no longer required to support the monitoring and manipulation of representations in working memory for response modulation. This would explain why the inhibitory effect of the IFG on the DLPFC becomes stronger during emotion regulation” (p. 1934).

Emotions can be made less intense by coming up with a Teacher theory that downplays existing mental networks, while emotions can be made more intense by coming up with a theory that reinforces existing mental networks. "Our results show that the successful reframing of negative stimuli in a positive direction (i.e., decreasing emotions to feel less negative affect) was related to less effective coupling between IFG and VMPFC. In contrast, the successful reframing of aversive pictures in a negative direction (i.e., increasing emotions to feel more negative affect) was associated with enhanced effective connectivity between IFG and VMPFC” (Morawetz, 2016b, p. 582). (This paper also focused upon the left IFG.)

Cognitive reappraisal has to be repeated in order to have a long-term impact, and this long-term impact will alter the way that the amygdala responds to emotional situations: "One week after successfully using cognitive reappraisal to diminish behavioral (negative affect) and neural (right amygdala) markers of emotional response, we found that the amygdala's response remained attenuated for images that had been reappraised four times, but not for images that had been reappraised only once. Critically, we found no evidence that these enduring changes in amygdala response required on-going recruitment of prefrontal regions involved in top-down control” (Denny, 2015, p. 11). (This experiment used unpleasant images that activated the right amygdala.)

Reappraisal is different than suppression: "Emotional perception can be regulated in different ways. Classic psychological models of emotion regulation mainly distinguish between a reappraisal strategy, which consists of reducing the emotional response by reinterpreting a situation, and suppression 
strategy, where expressive or bodily responses are inhibited by cognitive control” (Guex, 2019, p. 211). Reappraisal changes the response of the amygdala, while suppression attempts to control the amygdala after it responds: "Here we record amygdala activity from six patients undergoing surgery for pharmaco-resistant epilepsy during both reappraisal and suppression. We find that emotion reappraisal strategy, but not suppression, modulates early neural responses to emotional scenes during an extended period of time, starting $130 \mathrm{~ms}$ post-stimulus onset” (p. 211). Suppression is associated with Perceiver thought in the right dorsolateral frontal. For instance, when telling people not to think about pictures, "Successful retrieval suppression was associated with a right DLPFC-mediated top-down inhibition of visual imagery” (Smith, 2018, p. 105).

Study of personality indicates that Perceiver thought can respond to unwanted Mercy mental networks in one of two ways. One way is to try to suppress the unwanted mental network. This typically works for a while, but often ends in some outburst of anger, typically triggered by some event. The other way is to use Perceiver facts to come up with a Teacher understanding of the experiences within Mercy thought. This second method of cognitive reappraisal takes more work, but it is also more effective in the long-term.

This relates to the earlier discussion describing two ways that Perceiver thought can affect Teacher theories. First, Perceiver thought can point out facts that contradict theories. This leads to an adversarial relationship between Perceiver thought and Teacher thought, because Perceiver thought is limiting Teacher overgeneralization, while Teacher thought is attempting to avoid Perceiver facts. Similarly, suppression leads to an adversarial relationship between Perceiver thought and Mercy thought, because Perceiver thought is trying to avoid strong emotions while Mercy thought is attempting to focus upon emotional experiences. Second, Perceiver thought can make Teacher theories more general by building factual connections. This leads to cooperation between Perceiver thought and Teacher thought, because both are following the same goal of increasing Teacher emotions. Similarly, cognitive reappraisal leads to cooperation between Perceiver thought and Mercy thought, because both are following a similar strategy of seeking pleasant emotions. Going further, using facts to extend a theory can be combined with cognitive reappraisal, leading to cooperation between Perceiver thought, Teacher thought, and Mercy thought. And we saw earlier when looking at the left dorsolateral frontal that Server thought plays a role in escaping from depression. Thus, cognitive reappraisal becomes more effective when one attempts to understand how a situation can be changed and then reinforces this understanding with actions. All four of the simple cognitive modules are now cooperating, which means that one has now made significant progress towards the ultimate goal of mental wholeness that was mentioned earlier.

Turning now to the topic of cognitive development, mental symmetry suggests that the childish mind needs to be transformed because it is built upon an inadequate set of mental networks acquired from the physical body and from authority figures. As we have just seen, cognitive reappraisal is a major component of the process of personal transformation. Personal transformation is mentally possible because there are two kinds of emotion. Constructing a general theory about personal identity creates positive Teacher emotions, which make it emotionally possible to endure the emotional discomfort of reprogramming MMNs of childish thought. 
Cognitive reappraisal is similar to the scientific method. But reappraisal is not the same as scientific thought. That is because cognitive reappraisal is emotionally more fundamental than normal scientific thought. As Thomas Kuhn pointed out, "The scientific enterprise as a whole does from time to time prove useful, open up new territory, display order, and test long-accepted belief. Nevertheless, the individual engaged on a normal research problem is almost never doing any one of these things. Once engaged, his motivation is of a rather different sort. What then challenges him is the conviction that, if only he is skilful enough, he will succeed in solving a puzzle that no one before has solved or solved so well. Many of the greatest scientific minds have devoted all of their professional attention to demanding puzzles of this sort” (Kuhn, 1970, p. 38).

Cognitive reappraisal forms new theories in order to rethink emotional experiences. This formation of new theories in Teacher thought falls into what Thomas Kuhn referred to as revolutionary science with its paradigm shifts. In contrast, Kuhn's normal science uses a form of technical thinking that solves intellectual puzzles within some paradigm. This does not mean that a scientist is never coming up with hypotheses. Many scientific papers propose hypotheses which are then tested through some experiment. But the vast majority of these hypotheses involve small shifts of understanding within the framework of some general paradigm.

Turning now to the big picture, mental symmetry suggests that the mind can use both abstract and concrete thought in one of three primary ways: mental networks, normal thought, and technical thought. Mental networks involve Teacher and Mercy thought. Normal thought is guided by connections, analogies, and similarities provided by Perceiver and Server thought. Cognitive reappraisal typically uses the analogies of normal thought to reprogram mental networks. Technical thought, in contrast, emerges when Contributor thought restricts the mind to the 'playing field' of some limited set of well-defined rules, which Kuhn described as solving puzzles.

The focus of this paper so far has been upon the four simple styles. We will now examine the three composite styles. This will include a discussion of technical thought and how it interacts with mental networks and normal thought.

\section{Basal Ganglia}

The brain contains a loop that starts from the cortex, goes through the basal ganglia, and then returns through the thalamus on its way back to the cortex. Similarly, the diagram of mental symmetry shows three cognitive modules connected by arrows: Exhorter to Contributor to Facilitator. Thirty years ago, when I first looked at neurology, it seemed obvious that there was a correspondence between these two, with Exhorter and Contributor thought using the basal ganglia and the Facilitator using the thalamus. However, it did not make sense how two modes of thought could both share the basal ganglia, and not enough was known about the basal ganglia to tie down any of the details.

Much more is now known about the basal ganglia, and mental symmetry suggests the following general interpretation:

- Mental networks affect the emotional bottom line or paradigm via the ventral striatum and the striosomes. 
- Exhorter thought suggests some possibility to Contributor thought through the direct path, with alternatives coming through the indirect path.

- Contributor thought either accepts the suggestion provided by Exhorter thought or else chooses one of the alternatives.

- Contributor thought can also shut down activity or proceed in a cautious manner, using the hyperdirect path through the subthalamus.

- Facilitator thought then uses the thalamus to adjust and mix internal thought with sensory input in the light of the decision made by Contributor thought.

Turning now to the details, dopamine has traditionally been viewed as a chemical of pleasure. However, Berridge discovered that dopamine is related to 'wanting' and not to 'liking': “The brain circuitry that mediates the psychological process of 'wanting' a particular reward is dissociable from circuitry that mediates the degree to which it is 'liked'. Incentive salience or 'wanting', a form of motivation, is generated by large and robust neural systems that include mesolimbic dopamine. By comparison, 'liking', or the actual pleasurable impact of reward consumption, is mediated by smaller and fragile neural systems, and is not dependent on dopamine” (Berridge, 2016, p. 670).

Dopamine appears to be related to the activity of Exhorter thought. All addictive behavior appears to involve dopamine, and Exhorter strategy is the part of the mind that pushes and prods. Using the language of mental symmetry, Mercy and Teacher thought with their mental networks generate emotions or 'liking'. Exhorter thought, in contrast, uses these emotional memories as a starting point for generating motivation or 'wanting'.

Going further, the Exhorter person typically pushes others to make decisions, suggesting that the dopamine circuit is conscious while choice is subconscious. In contrast, the Contributor person often feels strongly driven while wanting to stay in control, suggesting that the dopamine circuit is subconscious while choice is conscious.

Putting this together, the source of motivation is some emotional 'bottom line'. In the right hemisphere, the bottom line comes from MMNs that represent objects, experiences, or persons, often involving money, fame, or fortune. In the left hemisphere, the 'bottom line' expresses itself as the TMN of some theory that is being expanded, or some system of structure or order that is being optimized. In the diagram of mental symmetry, Exhorter connects Mercy and Teacher and an arrow leads from Exhorter to Contributor. This indicates that mental networks within Teacher and Mercy thought provide the raw material for Exhorter drive, which then provides the motivation for Contributor choice.

The immature Exhorter person can be a 'party animal', finding excitement in sensory gratification. In contrast, the mature Exhorter person develops hope, focusing upon long-term goals. These two alternatives can be seen in dopamine circuits: "We propose a model in which dopamine (DA) can favor NOW processes through phasic signaling in reward circuits or LATER processes through tonic signaling in control circuits. At the same time, through its modulation of the orbitofrontal cortex, which processes salience attribution, DA also enables shifting from NOW to LATER, while its modulation of the insula, which processes interoceptive information, influences the probability of selecting NOW vs LATER actions on the basis of an individual's physiological state” (Volkow, 2016, p. 1). Notice the 
balance between orbitofrontal and insula. Recent research has shown that the insula represents self from a physical perspective. In contrast, the orbitofrontal contains an internal model of self based upon mental networks. Continuing with this paper, dopamine has both a short-term effect and a long-term impact: "NOW vs LATER processes are differentially modulated by DA: LATER processes require steady DA signaling in striatal and prefrontal regions to sustain effort as achieved by tonic DA firing, whereas NOW processes are predominantly driven by fast, sharp bursts of DA as achieved by phasic DA firing that drive attention to the salient stimulus. In humans, brain imaging studies have revealed that stimuli that result in fast DA increases in ventral and dorsal striatal regions are experienced as rewarding and generate the desire to attain the stimulus whereas stimuli that trigger slow and steady DA increases do not. By contrast, steady DA increases are associated with increases in the ability to exert sustained (i.e., cognitive) effort and to experience the task or stimulus as motivating and interesting” (p. 1). Thus, immediate sensory gratification is related to the insula and produces a short burst of dopamine. In contrast, long-term effort is related to the orbitofrontal cortex and long-term levels of dopamine. Both of these are characteristics of the Exhorter person, who can pursue immediate excitement, but can also continue relentlessly with some project over the long-term.

Turning now to the relationship between Exhorter motivation and Contributor choice, there is a direct path and an indirect path through the basal ganglia. The direct path is modulated by D1 dopamine receptors and the indirect path by $D 2$ dopamine receptors: "Basal ganglia circuitry comprises two major pathways linking input (striatum) and output (SNr and GPi): a monosynaptic GABAergic projection from dopamine D1 receptor-expressing striatal projection neurons (dSPNs) to the output nuclei including substantia nigra pars reticulata (SNr), called 'direct pathway'; and a polysynaptic projection from dopamine D2 receptor-expressing striatal projection neurons (iSPNs) to the output nuclei through external globus pallidus (GP) via subthalamic nucleus (STN), called 'indirect pathway'” (Jin, 2015, p. 6).

It was previously thought that the direct path facilitates action while the indirect path suppresses action. It is now known that choosing some option involves both of these paths: Jin explains that "These data suggest that direct pathway neurons could function to select the desired motor program, while indirect pathway neurons would inhibit the competing motor programs; co-activation of these pathways would allow the appropriate action selection" (p. 6). This is consistent with the way that Contributor choice functions. Contributor persons often like to think that they are able to choose anything. But such libertarian free will does not exist. Instead, study of personality indicates that Contributor thought chooses between a limited set of options, similar to an election in which one is presented with a list of political candidates and then chooses between them. Neurologically speaking, the direct path suggests some choice to Contributor thought while the indirect path provides a few alternatives. Contributor thought then chooses between the recommended choice and the alternatives. This definition of choice can be seen in the behavior of the Contributor person, who often exercises free will by choosing not to follow the recommended option.

This concept of Contributor free will is described more clearly in another paper that looked at dopamine in the human brain: "A new model of dopamine function in the basal ganglia posit that D1 receptor activation prepares a set of possible responses, then D2 receptor activation functions in selecting the final response. The present findings are consistent with such an integrated function” 
(Robertson, 2015, p. 5994). Dopamine activity is related to Exhorter thought. Thus, D1 appears to be related more to Exhorter urges, while D2 is connected with Contributor choices. Consistent with this, another paper "dissected the contributions of each pathway and found that both the direct striatonigral pathway and the indirect striatopallidal pathway are necessary for smooth initiation and the execution of learned action sequences" (Tecuapetla, 2016, p. 703). The authors conclude that "These observations are consistent with recent studies proposing that the direct pathway is more related to exploitative behavior, while the STN-GP complex is more involved in action shifting or exploration” (p. 713). (STN-GP stands for subthalamus and globus pallidus, which are part of the indirect pathway.)

Another paper adds some details: "We show that negative feedback learning can be modulated through D2 receptor signaling and positive feedback learning through D1 receptor signaling in the ventral striatum. Furthermore, stimulation of D2 receptors in the ventral or dorsolateral (but not dorsomedial) striatum promoted explorative choice behavior, suggesting an additional function of dopamine in these areas in value-based decision making” (Verharen, 2019, p. 1). In other words, if everything is going as expected, then it makes sense for Contributor thought to stick with the existing plan. But if problems arise, then it makes sense to pursue alternative paths. This differential effect of D1 and D2 also happens within the frontal cortex: "Risk/reward decision making is modulated differentially by these two receptors within the prefrontal cortex (PFC), with D2 receptors enabling flexible decision making and D1 receptors promoting persistence in choice biases" (Jenni, 2017, p. 6200). And this same conclusion is backed up by another paper: "These differential effects of DA D1 and D2 receptor antagonists on performance of a free choice task with probabilistic reward strongly suggest that direct pathway D1 receptor signaling promotes behavioral stability to attain high-value rewards while indirect pathway D2 receptor signaling facilitates switching away from smaller reward outcomes... Therefore, D1 and D2 signaling mechanisms in direct and indirect basal ganglia pathways appear to act cooperatively to optimize reward by regulating the balance between stable and flexible responses under conditions of probabilistic reward contingencies” (Ueda, 2017, pp. 6-7).

Graybiel has done extensive work examining how habits are formed within the basal ganglia of rodents and has discovered that forming a habit can be divided into three distinct stages with each emphasizing a different brain region: "The ventral striatum is necessary for initial learning of motivated behaviors that could become habitual. The dorsal striatum then becomes critical. First, behaviors are driven largely by the anticipated outcome of the behavior itself; this process, according to rodent lesion studies, requires the dorsomedial striatum ([DMS] in rodents). But then, according to these lesion studies, as the behaviors are repeated and bring about a positive outcome, the DMS is no longer required, but the dorsolateral parts of the striatum ([DLS] in rodents) is required for habitual performance” (Graybiel, 2018, p. 4). (The rat DMS is the equivalent of the human caudate, while the rat DLS corresponds to the human putamen. Both of these are part of the dorsal striatum.)

Putting this into cognitive terms, the first stage of forming a habit uses Exhorter thought, the second stage is guided by the goal-oriented thinking of Contributor thought, while the third stage moves into habitual action. Quoting further from the Graybiel paper, the first stage involves dopamine, which is related to Exhorter thought: "Much evidence suggests that the midbrain dopamine system, particularly the VTA, is influential in the initial stages of generation of these behaviors, and that the striatal target of the VTA system, which largely lies in the ventral striatum, is essential for the neural changes, leading to 
addiction” (p. 4). (VTA is ventral tegmental area, which is part of the ventral striatum.) The third stage is habitual and is no longer driven by goals: "There is a critical transition period during habit formation. Before this transition, a given behavior being learned remains sensitive to outcome (usually tested as sensitivity to reward value). But, after this period, the same behavior becomes independent of the reward value” (p. 4).

Graybiel adds that one critical aspect of learning a habit is a chunking of neuron activity: “As animals learn to run in maze tasks, the striatal activity, at first, marks the full run time, but later begins to bracket the entire run. Activity becomes more and more prominent at the beginning and end of the runs, or beginning and end of the action through the turns” (p. 6). In other words, a habit is a series of steps that the mind treats as a single unit. Once the mental chunk of a habit has formed in the putamen, then it is very resistant to change: "The taskbracketing pattern in the DLS is extremely resistant to degradation - it takes wholesale removal of rewards to block it fully, and even then, the pattern is latent, but not gone, and is rapidly retrievable” (p. 7). Tying this to the behavior of the Contributor person, on the one hand, the Contributor person is driven by some bottom line, but on the other hand, a Contributor person may perform habitual repetitive movements for years (such as selling hamburgers on a food truck), as long as this satisfies some financial bottom line in a manner that remains free of the control of others.

During the third stage of habit, dopamine becomes activated by the cues that trigger the habit, which indicates that mental networks are now involved. Quoting further from Graybiel: “Addicts experiencing craving to any one of a number of drugs can show a significant dopamine increase in striatum in response to drug-conditioned cues (such as thoughts leading to craving) with response levels that can be greater than those to the drug themselves. These cue-induced responses are particularly prominent in the dorsal striatum, including in the putamen” (p. 5). This transition from Exhorter excitement to Exhorter craving may be similar to the transition from NOW to LATER mentioned earlier, which implies that being driven internally by mental networks can be either beneficial or harmful, depending upon the nature of the mental networks.

One can tell that mental networks have become involved because the formation of a habit, as well as carrying out a habit, are ultimately under the control of the orbitomedial frontal cortex. Graybiel explains that "The medial prefrontal cortical region, called infralimbic (IL) cortex, in rodents, like the DLS, is necessary for habits to be performed. New optogenetic studies have shown that the IL exerts online control of the performance of well-ingrained habits and is necessary for their formation. This work is critical to any account of the role of the striatum in habit formation, as it suggests a form of cortical control that can, on a moment-by-moment basis, determine whether a behavior is performed habitually or not” (p. 10). (The rat infralimbic cortex is probably the equivalent of area 25, the subgenual anterior cingulate, which is at the bottom of the medial frontal near the orbitofrontal.)

This transition from habit to habit-backed-up-by-mental-network is described explicitly by Graybiel in another paper: "Even though we saw the beginning-and-end pileup of activity in the habit striatum, during the initial learning period we saw very little change in the infralimbic cortex. It was not until the animals had been trained for a long time and the habit became fixed that the infralimbic activity changed. Strikingly, when it did, a chunking pattern then developed there, too. It was as though the infralimbic cortex was the wise one, waiting until the striatal evaluation system had fully decided that 
the behavior was a keeper before committing the larger brain to it” (Graybiel, 2014, p. 42). Notice the progression. As the rat continues some behavior, it turns into a habit within the dorsolateral striatum and becomes treated as a single chunk of memorized activity. The infralimbic cortex then steps in and treats this habitual chunk of behavior as a mental network. Looking at this cognitively, it is possible that chunking may lead naturally to the formation of a TMN because a complex set of steps are being represented as a single chunk of behavior; this is a form of order-within-complexity.

Rats are not capable of abstract thought and they equate action with imagination. Therefore, all habits in a rat would involve physical actions. Presumably, human habits would extend to mental habits involving both concrete and abstract thought. Graybiel suggests something similar: "Habits of thought, at least in humans, are probably as common as motor habits and, like motor habits, are vulnerable to pathologic distortion. Our view is that such habits of mind can be created by cognitive pattern generators much as habits of action are generated" (Graybiel, 2018, p. 11). We looked previously at mental networks forming out of emotional memories. We see here that mental networks can also form out of habitual actions. This suggests that a mental network can contain various kinds of mental content. The common thread is that a mental network will use emotional pressure to impose its content - whatever that content is - upon the mind when it is triggered.

The TMN of a paradigm appears to form in a manner that is similar to the mental network driving some habit. Abstract technical thought is driven by the emotional goal of improving some Teacher theory. Study of personality shows that if a person continues to use a paradigm for a sufficient time, then this paradigm will turn into a Teacher mental network (TMN), which will then exert emotional pressure upon a person to continue using this paradigm and to defend this paradigm when it is threatened. (I am defining a paradigm as the TMN that lies behind some technical specialization, consistent with Thomas Kuhn's description of paradigms. Other forms of order-within-complexity can also turn into TMNs.)

Saying this more clearly, the TMN of a paradigm will not be emotionally apparent when technical thought is being used within this paradigm, because the TMN is continuing to encounter content that is consistent with its structure. Instead, thought will appear to be coldly rational. However, if the paradigm is questioned or threatened in some manner, then this will face the TMN with incompatible data which will provoke an emotional response, typically in the form of belittling or sarcasm. I suggest that sarcasm could be defined as emotional belittling conveyed within a package of technical thought.

The three stages of forming a habit are also consistent with the concept of free will that is suggested by mental symmetry. The average person thinks that free will is being exercised when a person makes a choice in some major crisis. However, how a person responds in crisis is usually determined by the mental networks that are being triggered and the habits that they provoke. The actual free will is happening in the previous stage when choices are being made to pursue goals, and habits are being formed. Saying this more simply, free will does not usually occur in a crisis, but is rather exhibited primarily in the many little choices that one makes before a crisis, which will predetermine how a person responds in a crisis. Thus, a crisis usually reveals a person's character by bringing core mental networks to light. Concluding, free will is real, but it is limited, and it usually happens before one thinks that it does. This does not mean that there is no free will in a major crisis. There is always some free will, but free will by itself can only be used to override minor mental networks and it occurs within 
a context that is determined by core mental networks. Using an analogy, free will can be used to choose how one will respond to a question, while core mental networks will determine the question that is being asked.

\section{Striosomes}

Recent research has clarified the role played by the striosomes. Striosomes are patches within the basal ganglia that have different structure and connectivity than the matrix surrounding the patches. The striosomes appear to be related to Exhorter thought: First, "striosomes in the anterior striatum have strong inputs from regions related to the limbic system, including parts of the orbitofrontal and medial prefrontal cortex" (Bloem, 2017,p. 2). The limbic areas are related to Teacher and Mercy thought, and Exhorter connects Teacher and Mercy. Second, "striosomes are equally specialized in their outputs: they project directly to the dopamine-containing neurons of the substantia nigra” (p. 3). Dopamine is related to Exhorter thought.

Graybiel's lab examined the relationship between the striosomes and the matrix by comparing how two regions in the rat medial frontal cortex treated value: "One, originating in the prefrontal region called prelimbic cortex in rodents (here called PFC-PL), projects preferentially to striosomes in the associative striatum. The second, originating in the prefrontal region called in rodents anterior cingulate cortex (here named PFC-ACC), projects preferentially to the matrix compartment of the associative striatum” (Friedman, 2015, p. 1321). I should add that this paper studied the dorsomedial striatum, which performs goal-oriented behavior in the rat. The rat PFC-PL is probably the analog of the monkey and human perigenual anterior cingulate cortex. It is right above the rat infra-limbic frontal cortex mentioned earlier. Tying this together with what was said previously about the perigenual anterior cingulate, the part of the anterior cingulate that deals with personal identity connects with the striosomes in the basal ganglia, whereas the part of the anterior cingulate that handles value in a more objective manner connects with the matrix in the basal ganglia.

These two regions of the rat frontal cortex were inhibited one at a time: "The increased choice of the high-cost, high-reward option induced by inhibiting the PFC-PL pathway to striosomes thus appeared specific to the cost benefit conflict context, in which the animals had to accept substantial cost to gain reward and had to regulate their approach and avoidance behaviors. By contrast, inhibition of the predominantly matrix-targeting PFC-ACC pathway significantly affected the animals' choices in all tasks except the cost-cost task” (p. 1323). In the high-cost, high-reward option, the rat could get a sip of high quality chocolate milk, but had to undergo the cost of being shone on by a bright light. The striosomes come into play when dealing with such high value choices. The authors conclude "that a medial prefronto-striosomal circuit is selectively active in and causally necessary for cost-benefit decision-making under approach avoidance conflict conditions known to evoke anxiety in humans” (p. 1320).

Looking at this from a cognitive perspective, Contributor thought performs cost-benefit analysis, and we have seen that this is related to the anterior cingulate. A different kind of value analysis comes into play when dealing with strongly emotional subjects, which involves the perigenual anterior cingulate cortex. This can be seen in the Exhorter person, who is naturally drawn to emotional crises and may create one if it does not exist. In contrast, the Exhorter person is not naturally good at cost-benefit 
analysis. The interaction between these two kinds of value can be seen in the familiar tropes 'Would you sell your grandmother?' and 'Everyone has his price'. Selling involves Contributor cost-benefit. However, one's grandmother is associated with strong mental networks. Thus, at some point the price becomes too high for normal Contributor business transactions because one is threatening mental networks.

Speaking from personal experience, I have found that my decision-making changes when a situation becomes sufficiently emotional. I stop trying to optimize the situation and start thinking in terms of protecting core mental networks. When such a transition happens, it feels as if something is stepping forward within my mind and advising: 'Caution. This is not a normal situation. Be careful how you respond because it will affect core mental networks.' I will refer to these two as Contributor value and Exhorter value.

Friedman found that when Exhorter value is disabled, then Contributor value will be followed regardless of the cost to mental networks: "Intrastriatal inactivation of the striosome-targeting PFC-PL pathway strongly affected decision-making in the cost-benefit conflict task: the animals ran more toward the high-cost option” (p. 1323). "By contrast, inhibition of the predominantly matrix-targeting PFC-ACC pathway significantly affected the animals' choices in all tasks except the cost-cost task: the animals shifted their choices toward the option with higher reward” (p. 1323). Thus, if Contributor value is disabled, then all that is left is Exhorter excitement: Go for broke; get the best benefits. (In the cost-cost task, the benefit was the same, but the cost was different.)

A later paper from the same lab looked at the relationship between striosomes and stress: "The effect of chronic stress on decision-making was strikingly selective. The stressed animals were significantly more likely to choose high-cost/high-reward options than matched controls in the CBC task” (Friedman, 2017, p. 1192). (CBC is cost-benefit conflict. This paper also focused on the DMS or dorsomedial striatum in the rat.) In other words, stress puts the mind into crisis mode and leaves Exhorter thought in charge of decisions. This explains why the Exhorter person is attracted to crises and may actually create a crisis if needed.

The Exhorter person is not naturally subtle, but instead tends to make large movements and come up with major decisions. Saying this more carefully, Contributor thought performs cost-benefit analysis guided by mental networks. Exhorter thought performs major changes in which mental networks themselves are altered. (This is similar to Thomas Kuhn's distinction between normal science and revolutionary science.) Relating this to the striosomes, "a primary long-term effect of chronic stress is a shift in circuit dynamics, leading to a shift in the E-I balance of the cortico-striosomal circuit toward abnormal excitation of striosomes” (p. 1202). (E-I balance is excitation versus inhibition.) Contributor thought uses logic to evaluate choices. In contrast, "Chronic stress selectively affected choices in the CBC task. The utility functions of the animals were profoundly affected, so that the stressed rats and mice maintained approaches to high-cost/high-reward choices over a wide range of options, seemingly failing to make a normal, 'rational' transition away from approaching the normally avoided option” (p. 1203).

Another author looked at this same pre-limbic frontal cortex in the rat: "Inspired by bandit tasks for humans, we trained rats to choose freely and without cue guidance between a certain-small reward on 
the 'safe-arm' of a Y-maze or a possible-big reward on the 'gamble-arm”” (Passecker, 2019, p. 153). "Strikingly, a major proportion of recorded neurons in the prelimbic cortex significantly increased their firing during the experience of no-reward at the gamble-arm” (p. 153). Describing this in more detail, "The predictive firing-rate increase of choice-predicting cells precedes an upcoming change to the safe arm on the next trial by several seconds and is highly time restricted to the encounter of no-reward” (p. 161). In other words, the rat decided to follow Exhorter value and go for the potential big reward. But when this reward did not happen, then the motivating mental network experienced input that was inconsistent with its structure, leading to negative feelings. This happened to the rat that experienced no-reward at the gamble-arm. When a mental network generates negative feelings, then this emotional pain can be eliminated by giving the mental network what it wants. For the rat, this meant visiting the safe-arm to get a reward, even if this reward was small.

Graybiel's lab has recently extended their work to the monkey. The human and monkey analog to the prelimbic rat frontal region is the perigenual anterior cingulate (pACC). "The presumed rodent homologue of the pACC has been implicated in circuits related to the striosome compartment of the striatum" (Amemori, 2019, p. 3). "These findings are the first to demonstrate that cortical sites in nonhuman primates behaviorally identified as modulating conflict decision-making send corticostriatal innervations favoring the striosome compartment over the surrounding matrix" (p. 6). "Our results in non-human primates provide causal evidence that circumscribed, behaviorally identified zones in the pACC and cOFC participate in corticostriatal circuits that contribute critically to negative decisionmaking under challenging cost-benefit decision-making” (p. 7). In simple language, this region also lights up in monkey brains and indicates 'Do not sell your grandmother. She is too valuable.'

Kolling has looked at the role that the perigenual anterior cingulate plays in determining value in the human mind. He distinguishes between myopic and prospective value: "The overall value of the environment (sometimes called 'search value') can be decomposed into myopic and prospective components. The myopic component corresponds to the average benefits that might immediately follow a decision while prospective value corresponds to future benefits that might accrue over the longer term by taking a particular choice now" (Kolling, 2018, p. 1069). The authors "found that perigenual ACC (pgACC) was among the regions in which there was more activity during decision making (decision phase main effect) in participants who used prospective value more to guide their behavior. In other words, overall decision activity in pgACC is predictive of the degree to which prospective value will drive participants’ behavior” (p. 1078). Myopic value can be calculated using Contributor knowledge of cost-benefit. Prospective value extends into the realm of mental networks, asking questions such as 'Can I live with this choice?' or 'Is this choice a good fit with my personality?' For instance, selling one's grandmother may be profitable in the short term, but in the long term it will lead to significant discomfort involving mental networks.

One of the characteristics of a mental network is that it will use emotional pressure to impose its structure upon the mind when it is triggered. Similarly, Kolling found that "One notable bias exhibited by participants was a tendency to over-persevere; participants tended to search more than was optimal given the model-derived estimate of search value (appropriately adapted for progress through the sequence as the search horizon shrunk). Specifically, participants were more likely to search yet again as a function of how often they had already searched earlier in the sequence. Again, we found further 
support for this effect in the debriefing questionnaire, with participants reporting that they were aware that they sometimes searched more than they should because they had already searched on this trial” (p. 1073).

Prospective value also involves Perceiver facts and Server sequences, because these describe long-term consequences: "Prospective value, unlike myopic value, was an important determinant of the strength of interactions between dACC and dIPFC. These interactions occurred at an early point in the decision process and peaked when the increase in prospective value (temporal derivative of prospective value) peaked” (p. 1080). Thus, the dorsal anterior cingulate, which calculates normal, myopic Contributor value, is also active when calculating prospective value. But it retrieves facts and sequences from the dorsolateral prefrontal in order to look beyond the immediate situation to long-term consequences.

In addition, prospective value engages Exhorter effort. This relates to the distinction between NOW and LATER mentioned earlier. In the words of Kolling, "PgACC, partly through its interactions with ventral striatum, is important for motivating effortful sequential search decisions over time. In contrast to dACC, which carried strong signals related to the planning of an extended behavioral sequence, pgACC activity was more closely related to the motivation to engage in the sequence” (p. 1080). This relates to another trait of the Exhorter person. I mentioned that the immature Exhorter person can be a party animal, going from one excitement to another. The mature Exhorter, in contrast, will encourage people to 'stick with it', and will tend to pursue the same option with increasing force when encountering opposition. (The partially mature Exhorter will verbally exhort others to 'hang in there' while himself moving on to greener pastures.) This Exhorter component is associated with striosomes within the ventral striatum: "Experiments with both humans and rats, in tandem with the present results, suggest pgACC exerts its influence on behavioral motivation via interactions with striosomes, which are prominent in the ventral striatum, through a well-described anatomical circuit” (p. 1080).

Summarizing, we we have just examined how goals are evaluated in the rat dorsomedial striatum (DMS), the part of the basal ganglia that deals with goal-oriented behavior, comparing the paralimbic frontal cortex (FPC-PL) that projects to the striosomes with the anterior cingulate that projects to the matrix. We looked previously at the infralimbic frontal (FPC-IL) and the dorsolateral striatum (DLS), the regions that deal with habits. The infralimbic frontal cortex also projects primarily to the striosomes: "Although the role of the infralimbic-striosome system in habit formation is not clear, it may in fact be engaged in Pavlovian control of instrumental systems.” (Yin, 2006, p. 474). These systems do not function in isolation from one another. Instead, the mind mixes and matches between habits and goal-oriented behavior: "The computational processes underlying forward planning are expressed in the anterior caudate nucleus as values of individual branching steps in a decision tree. In contrast, values represented in the putamen pertain solely to values learnt during extensive training. During actual choice, both striatal areas show a functional coupling to ventromedial prefrontal cortex, consistent with this region acting as a value comparator. Our findings point towards an architecture of choice in which segregated value systems operate in parallel in the striatum for planning and extensively trained choices, with medial prefrontal cortex integrating their outputs” (Wunderlich, 2012. p. 786).

Tying this in with previous papers, Wunderlich used brain imaging on humans. The caudate is the human equivalent of the rat DMS, which performs goal-oriented behavior, while the putamen is the 
human equivalent of the rat DLS, which carries out habits. This study had people perform tasks in which they could choose between goal-oriented steps and habitual actions. The corresponding part of the basal ganglia was activated depending upon whether a step was goal-oriented or habitual. These different kinds of steps are coordinated by the ventromedial prefrontal cortex. (The ventromedial prefrontal is a somewhat vague term that would presumably include the human equivalent of the rat paralimbic and the infralimbic, as well as much of the orbitofrontal.) Looking at this cognitively, mental networks and their connections are stored within the ventromedial prefrontal cortex, and this region appears to choose which mental networks will be activated. But a mental network can be composed of different kinds of elements, some of which are more habitual, while others are more goaloriented.

\section{Ventral Striatum}

Our discussion of the basal ganglia has focused so far upon what is known as the dorsal striatum. The monkey and human dorsal striatum are composed of the caudate, which is related to goal-oriented behavior, and the putamen, which is responsible for habits. The ventral striatum is composed of the nucleus accumbens, together with a poorly defined region extending beyond the bottom of the caudate and putamen. (The basal ganglia are composed of the striatum, as well as the globus pallidus, substantia nigra, and subthalamus.) My general hypothesis is that dopamine and the ventral striatum are connected with Exhorter thought.

I have mentioned that Exhorter thought exerts emotional pressure to push the mind in a certain direction. Contributor thought then chooses either to follow this emotional pressure or else pick some related alternative. This pushing and prodding of Exhorter thought is carried out by the nucleus accumbens: "A main function of the NAc is to bias the direction and/or intensity of behavior, thereby increasing the likelihood that certain actions are committed. This function is likely mediated through output pathways from the NAc that ultimately feed into motor systems and nudge behavior in certain directions" (Floresco, 2015, pp. 34-35). The diagram of mental symmetry shows Exhorter going from Teacher and Mercy to Contributor. This is reflected in the connections of the nucleus accumbens: "Owing in part to its neuroanatomical connectivity, the NAc has been proposed to integrate mnemonic and emotional signals from nodes within the limbic system residing in the frontal and temporal lobes that determine the response priorities of an organism. In turn, the NAc is thought to integrate these signals and turn them into action via output to pallidal and other subcortical motor effector sites through which signal outflow from this region may bias the direction and intensity of behavior” (p. 26). Continuing with this paper, the nucleus accumbens can be subdivided into a 'core' and a 'shell'. The core provides motivation, while the shell eliminates distraction: "Although considerable evidence suggests the NAc core mediates a type of 'go' response toward motivationally relevant stimuli, accumulating evidence suggests that the shell suppresses certain patterns of behavior that may interfere with goal seeking” (p. 37). Both of these traits are related to Exhorter thought. The Exhorter person will typically focus upon some goal to the exclusion of other alternatives, conveying the impression that the current focus is the most important thing in the world while every other focus is insignificant.

The Exhorter person has a natural tendency to exaggerate. As a result, the Perceiver and Exhorter persons often regard each other with suspicion; the Perceiver person thinks that the Exhorter person is 
being loose with the facts, while the Exhorter person thinks that the Perceiver person is trying to squelch the vision. This kind of interaction occurs between the nucleus accumbens and the hippocampus. Quoting further from Floresco: "When viewing these findings in light of those implicating the NAc shell in suppressing goal-irrelevant behaviors, one potential interpretation is that the shell may refine search behavior guided by the hippocampus by suppressing the tendency to approach locations known not to be associated with reward” (p. 39). Using cognitive language, the left and right hippocampi perform Server and Perceiver processing. Perceiver facts (and presumably Server sequences) tell Exhorter thought which areas have already been explored. Thus, “disconnection of this circuit caused rats to enter the maze arms with equal frequency, regardless of whether the arm was paired with food” (p. 39). In other words, Exhorter thought in the rat is excited by the prospect of food and Perceiver thought is responding, 'Do not go here. You already explored this area and you know that there is no more food.'

One cognitive byproduct of this is that Exhorter thought will naturally be attracted to alternatives where Perceiver facts and Server sequences are weak. Applying this principle to abstract thought, Exhorter thought tends to exaggerate when following some emotional vision or theory where Perceiver and Server knowledge is limited, because the factual uncertainty makes it possible to increase Teacher feelings of generality as well as Mercy feelings of personal importance. We saw this interaction when looking at overgeneralization.

This interaction between Exhorter drive and Perceiver knowledge occurs at the more general level of risk versus caution: "RDLPFC activity mediated less risky decision-making while VST mediated more risky decision-making across drug users and controls” (Yamamato, 2015, p. 2). More specifically, "stimulation of RDLPFC increased risk avoidance and reduced drug cravings in addicts while suppression of RDLPFC activity was associated with riskier decision-making” (p.2). In contrast, "VST activity was associated with decreased risk avoidance. Impulsive decisions have been associated with VST activity in healthy subjects” (p. 9). (RDLPFC is right dorsolateral prefrontal cortex, which is related to Perceiver thought, while VST means ventral striatum.)

Looking at this from a different perspective, the ventral striatum appears to be the core of a mental system motivated by discovery, which one paper refers to as 'the seeking system': "The SEEKING system is believed to function as an objectless appetitive system — a 'goad without a goal'-until the exploratory disposition it produces leads to the discovery and learning of useful regularities... The core structures that comprise the SEEKING system in the rat are the ventral tegmental area (VTA), the nucleus accumbens (NAcc), the ventromedial prefrontal cortex (VMPFC), and the dopaminergic projections originating from the VTA that innervate these areas” (Domenico, 2017, p. 4). The authors suggest that this system is a 'goad without a goal'. This conclusion makes sense if only Mercy emotion exists. However, I suggest that exploration is being driven by an emotional goal, which is the discovery of Teacher order-within-complexity.

For instance, the initial research on mental symmetry was done by me, a Perceiver person, helping my older brother, a Teacher person. What typically happened was that my brother would come up with some new theory in an area where my Perceiver facts were weakest, and I would respond by learning more facts in this area. Saying this more carefully, Teacher thought finds general theories emotionally appealing. A lack of Perceiver facts makes it possible for Teacher thought to make sweeping 
overgeneralizations, prodded by Exhorter exaggeration. Perceiver thought wants factual certainty. Thus, I would respond to a new theory by learning Perceiver facts in this area. This would place factual limits upon my brother's new theory, who would then typically respond by coming up with another theory in some other area where our factual knowledge was uncertain. The end result was to explore the territory of human thought, and come up with a general theory containing order-within-complexity, consistent with the idea of a 'seeking system'.

Domenico defines this drive to explore as intrinsic motivation and contrasts this with goal-oriented behavior: "Intrinsic motivation refers to the spontaneous tendency 'to seek out novelty and challenges, to extend and exercise one's capacity, to explore, and to learn'. When intrinsically motivated, people engage in an activity because they find it interesting and inherently satisfying. By contrast, when extrinsically motivated, people engage in an activity to obtain some instrumentally separable consequence, such as the attainment of a reward, the avoidance of a punishment, or the achievement of some valued outcome” (p. 1).

Looking at this cognitively, goal-oriented behavior is driven by some emotional bottom line in Mercy thought. Intrinsic motivation can emerge from Teacher emotion either directly or indirectly. The direct method was just discussed: One explores some topic or region in order to acquire a general understanding characterized by Teacher order-within-complexity. The indirect method functions through Platonic forms. Mental symmetry suggests that Platonic forms emerge in the following manner: Perceiver thought organizes Mercy experiences into categories, such as the category of round objects. This 'complexity without order' will motivate Teacher thought to come up with a general theory that explains these categories, such as the definition of a circle. (We saw this two-stage process when looking at the anterior hippocampus.) This Teacher theory will then lead indirectly to an internal Mercy image that portrays the simplified essence of the original Mercy experiences, such as the internal Mercy image of a perfect circle. This internal image of simplified perfection is a Platonic form. Goal-oriented behavior becomes intrinsically motivated when specific goals are placed within a framework of Platonic forms. For instance, instead of just developing a new car, one is coming up with a car that resembles more closely one's internal concept of the ideal car.

The relationship between intrinsic motivation and exploration can be seen in monkeys. Domenico summarizes: “Long before Deci’s experiments concerning intrinsic motivation in humans and its undermining by rewards, Harlow documented this effect in rhesus monkeys. He coined the term intrinsic motivation to describe his observation that these primates would persist in playing with mechanical puzzles even in the absence of external rewards. Indeed, he observed that the introduction of rewards for playing led these primates to decrease their spontaneous manipulative explorations, relative to those not exposed to external rewards” (Domenico, 2017, p. 2).

Going further, the ventral striatum is related to meaningful novelty: "The magnitude of belief updates about task structure (meaningful information), but not pure sensory surprise (meaningless information), are encoded in midbrain and ventral striatum activity” (Nour, 2018, p. 1). And this is related to dopamine: "Dopamine is thought to play a central role in processing the meaningful information content of observations, which motivates an agent to update their beliefs about the environment” (p. 1). This can be seen in the Exhorter person. The Exhorter person usually ignores details, but the Exhorter person will focus upon details if they are important. Nour defines what it means for a detail to be 
important: "Meaningful information can be formally quantified as the degree to which a new observation changes an agent's prior belief about the current state of the world, given previous observations, to a new (posterior) belief” (p. 2). In other words, a detail is important if it changes the way that I think.

Mental symmetry uses the word 'belief' to refer to fundamental facts within Perceiver thought. In contrast, Nour uses 'belief' to refer to an internal expectation of what will happen. This expectation is stored within the medial frontal cortex and updated by the ventral striatum: "We found that belief updates were encoded in the medial frontal cortex, including dorsal anterior cingulate. This observation is consistent with previous human and nonhuman primate studies as well as with suggestions that anterior cingulate cortex is active in novel or volatile environments wherein agents need to refine their internal models in light of new observations... The ventral striatum and SN/VTA are implicated in encoding signed reward prediction errors that update action and state values” (p. 7-8). (SN/VTA means substantia nigra and ventral tegmental area, which are the primary sources of brain dopamine.) Noor relates this to schizophrenia: "The findings of our study are highly relevant for dopaminergic and neurocomputational theories of schizophrenia. The aberrant salience hypothesis proposes that symptoms such as paranoia arise when unwarranted meaning and behavioral salience is attributed to ambiguous, irrelevant, or unreliable stimuli” (p. 8). Putting this together, mental networks are stored in the orbitofrontal cortex. The medial frontal cortex interconnects these mental networks. The ventral striatum decides when some mental network needs to be updated in the light of some significant detail. Paranoia happens when mental networks are being updated in the light of details that are not actually significant.

For instance, I have mentioned doing research together with my older brother. My other brother suffers from schizophrenia. My schizophrenic brother would make comments such as 'That shirt is black. Black is evil. We need to get rid of that shirt.' In other words, he was treating the color black as a significant detail and responding at the level of changing mental networks. He felt that his mental network of the current environment had to be protected from being contaminated by his mental network of evil that was triggered by the color black.

We talked previously about Exhorter value. It appears that the ventral striatum is generating emotional urges guided by Exhorter value. This is discussed in a recent paper that tries to reconcile two different functions that have been ascribed to dopamine. The author poses the question, "Is dopamine a signal for learning, for motivation, or both?” (Berke, 2018, p. 1). “The point is that learning and motivation are conceptually, computationally, and behaviorally distinct - and yet dopamine seems to do both" (p. 3). The author says that the latest evidence contradicts the standard interpretation that long-term levels of dopamine (tonic dopamine) are related to motivation. That is because "there is no direct evidence that tonic dopamine cell firing normally varies over slow time scales. Tonic firing rates do not change with changing motivation” (p. 3). Instead, "using the finer temporal resolution still of voltammetry we observed a close relationship between sub-second dopamine fluctuations and motivation. As rats performed the sequence of actions needed to achieve rewards, dopamine rose higher and higher, reaching a peak just as they obtained the reward (and dropping rapidly as they consumed it)” (p. 4). (This may relate to Volkow's distinction between NOW and LATER, because dopamine seems to be providing both short-term and long-term motivation.) 
Berke suggests that dopamine is related to effort and resources: "Dopamine provides estimates of how worthwhile it is to expend a limited internal resource, with the particular resource differing between striatal subregions. For 'motor' striatum ( DLS) the resource is movement, which is limited because moving costs energy, and because many actions are incompatible with each other... For 'cognitive' striatum ( DMS) the resources are cognitive processes including attention (which is limited-capacity by definition) and working memory. Without dopamine, salient external cues that normally provoke orienting movements are neglected, as if considered less attention-worthy... For 'motivational' striatum ( NAc) one key limited resource may be the animal's time. Mesolimbic dopamine is not required when animals perform a simple, fixed action to rapidly obtain rewards” (p. 8).

Translating this into cognitive language, effort can be defined as the emotional cost involved either in continuing with some mental network or in changing from one mental network to another. Motivation is an Exhorter drive to continue with the same mental network. Learning is an Exhorter drive to follow some alternative mental network, often prompted by some significant detail, some 'salient external clue'. The relationship between these two can be seen in the behavior of the semi-mature Exhorter person. Such an individual will talk extensively about learning and encourage others to learn while often remaining stubborn and unteachable himself. However, if such an Exhorter person experiences failure, then this can lead to a short window of openness to learning.

A general Teacher theory makes it possible to combine motivation and learning. That is because one is being motivated to increase the order-within complexity of the theory through learning. The more general a theory, the more it becomes possible to combine motivation with learning. For instance, the theory of mental symmetry is a meta-theory that motivates me to learn about other specific theories. Going further, the order-within-complexity of a theory can be increased by applying this theory within the real world. Thus, abstract learning leads to concrete motivation, because concrete behavior becomes viewed as an illustration and development of the general theory. This suggests that there may be a sense in which tonic dopamine is related to motivation, because a general theory can provide motivation through dopamine for an extended period of time without Exhorter thought getting bored through habituation.

Domenico relates the idea of intrinsic motivation to flow: "Like intrinsic motivation, when people experience flow, the satisfactions they experience are inherent to the activity itself and their behavior is 'autotelic' (auto = self, telos = goal) or performed for its own sake” (Domenico, 2017, p. 4). Using the language of mental symmetry, flow can be defined as Exhorter thought being guided by some mental network without being distracted by other mental networks. One of the characteristics of flow is that one is not aware of the passage of time. When one is in a state of flow, one will look up at the clock and realize that several hours have passed. This relates to the suggestion made by Berke that the limited resource managed by the ventral striatum is time. This would refer to subjective time, which could be defined as how often one is being interrupted by competing mental networks. The more frequent the emotional interruptions, or the more that one's mind wanders, the slower the sense of subjective time.

One aspect of flow is optimal challenge. Researchers "describe the flow state as the subjective experience of engaging 'just-manageable challenges by tackling a series of goals, continuously processing feedback about progress, and adjusting action based on this feedback'” (p. 5). This can be seen in the Exhorter person. On the one hand, the Exhorter person detests being bored. Boredom means 
no change and no significant details. On the other hand, the Exhorter person also detests being frustrated. Frustration happens when pursuing the current mental network reaches a dead end. Flow lies in between these two extremes, in which there are a series of 'just-manageable challenges'.

Domenico mentions that one study "examined the neural correlates of flow by asking participants to work on mental arithmetic task and comparing [sic] experimentally challenging levels with boredom and overload conditions. Results indicated that flow states were associated with increased activity in the left putamen and left IFG, again implicating core regions of both the dopaminergic system and the central executive network" (p. 9). We saw earlier that the left inferior frontal gyrus is the cortical region that works out Teacher order-within-complexity. If the left IFG is active, then this indicates that flow is being guided by some general theory in Teacher thought. The left putamen would presumably be related to Server habits. The Exhorter person usually regards habits as boring; one Exhorter friend of my brother liked to say that 'a rut is a grave with the ends kicked out'.

Another study provides a possible explanation for the activity within the left putamen. The authors related D2 levels with flow and found that "There was a significant correlation between striatal D2receptor availability and flow proneness. An exploratory analysis of striatal subregions showed that the relation was mainly driven by the dorsal striatum, with a significantly higher correlation in the putamen than in the ventral striatum” (Manzano, 2013, p. 1). More specifically, D2 availability was highest in the putamen, fairly high in the caudate, and especially low in the left ventral stratium. If D2 means that alternate choices suggested by Exhorter thought are being chosen by Contributor thought, then flow implies a mindset that follows habits in a flexible manner, has some goal-oriented behavior, but does not question existing mental networks, especially the current TMN. This combination makes sense as an expression of 'optimal challenge'. When one is following habits, then each step has a low emotional cost, but when these habits are flexible, then one is still able to move from one habit to another in a flowing manner, especially if one does not have to question the paradigm that is guiding behavior.

For instance, the Civilization series of computer games are infamous for creating mental flow. One article that examined the development of this game explained: "Meier and the rest of the developers realized they had accidentally bottled lightning when early testers kept reporting that they were losing track of time while playing - they got the 'I looked up and it was 3am' story from so many testers that the development team put a concerted effort into trying to figure out exactly what they'd managed to create” (Hutchison, 2019). Looking at this briefly, one starts a game of Civilization with a single settler unit in an unexplored world and then gradually builds a civilization by performing various steps such as exploration, expansion, research, development, trade, diplomacy, or conquest. At the level of specifics, each of these steps is simple and can be performing in a habitual manner with little emotional cost. However, there is great flexibility in which step one chooses to perform next as well as the order in which one can perform these steps. In contrast, at the level of generality, all of these simple steps are aspects of the one general Teacher theory of 'building a civilization', and one must use Teacher thought extensively to assemble the various steps in a manner that expresses this general Teacher theory. The end result is that one continually feels driven to take 'just one more turn'. 


\section{Addiction}

A distinction needs to be made between flow and addiction. Flows functions within the constraints of some limited world, such as the simulated world of a game of Civilization. Technical thought often has the characteristic of flow. Addiction is much narrower, and appears to be related to inflexible habits backed up by potent mental networks in a manner that leaves no room for goal-oriented behavior. Thus, one could view the game of Civilization as addictive if one compares the narrow realm of a computer game with the wide world of physical reality. But there is still considerable room for goal-oriented behavior within a game of Civilization. Saying this more generally, there is room to apply technical thought within some specialization. One can also use normal thought to break out of the narrow realm of a game of Civilization by looking at the extensive parallels between real history and the simulated world within the game. Similarly, normal thought can be used to expand technical specializations by looking for parallels between one specialization and another. With true addiction, the goal-oriented behavior gets squeezed out, leaving only habits and mental networks.

We saw the transition from habit to habit-backed-up-by-mental-network earlier in a quote by Graybiel: "Even though we saw the beginning-and-end pileup of activity in the habit striatum, during the initial learning period we saw very little change in the infralimbic cortex. It was not until the animals had been trained for a long time and the habit became fixed that the infralimbic activity changed. Strikingly, when it did, a chunking pattern then developed there, too. It was as though the infralimbic cortex was the wise one, waiting until the striatal evaluation system had fully decided that the behavior was a keeper before committing the larger brain to it” (Graybiel, 2014, p. 42). Notice the progression. As the rat continues some behavior, it turns into a habit within the dorsolateral striatum and becomes treated as a single chunk of memorized activity. The infralimbic cortex then steps in and treats this habitual chunk of behavior as a mental network.

Notice also how Contributor goal-oriented behavior has become caught in the middle. The emotional strength of the mental network overwhelms Contributor decision, while the rigidity of the habit limits Contributor choices. This is like walking along a sidewalk and then suddenly being dragged by force into a car and driven to some destination.

Looking at this in more detail, the pushing and prodding of a habit comes from Exhorter thought, dopamine, and the ventral striatum. As was mentioned previously, Berridge initially discovered and promoted the idea that dopamine is related to 'wanting' rather than 'liking': "Many studies have now accumulated supporting our original conclusion that dopamine mediates desire rather than pleasure, and it is now rather rare to find an affective neuroscientist studying reward who still asserts that dopamine mediates pleasure 'liking'” (Berridge, 2017, p. 2). Berridge describes the connection between wanting, dopamine, mental networks, and the ventral striatum: “'Wanting' is mediated largely by brain mesocorticolimbic systems involving midbrain dopamine projections to forebrain targets, such as the nucleus accumbens and other parts of striatum. The intensity of the triggered urge depends both on the cue's reward association and on the current state of dopamine-related brain systems in an individual. This interaction allows 'wanting' peaks to be amplified by brain states that heighten dopamine reactivity, such as stress, emotional excitement, relevant appetites or intoxication” (p. 3). Summarizing, emotional intensity moves the mind away from Contributor value to Exhorter value, making a person more prone to be driven by mental networks, which are then turned into urges by 
Exhorter thought within the nucleus accumbens (part of the ventral striatum) through the help of dopamine.

The initial stages of addiction are characterized by goal-oriented behavior, in which Exhorter thought motivates Contributor thought to choose to pursue some desirable mental network. But addiction eventually turns into habits triggered by mental networks, in which cues from the environment trigger a mental network, causing an inflexible habit to be carried out independently of Contributor choice. In the words of Berridge, “Ordinarily, cognitive wanting and incentive salience 'wanting' go together, so that incentive salience can give heightened urgency to feelings of cognitive desire. But the two forms of wanting vs. 'wanting' can sometimes dissociate, so that incentive salience can occur either in opposition to a cognitive desire or even unconsciously in absence of any cognitive desire. Incentive salience 'wanting' in opposition to cognitive wanting, for example, occurs when a recovering addict has a genuine cognitive desire to abstain from taking drugs, but still 'wants' drugs, so relapses anyway when exposed to drug cues or during vivid imagery about them” (p. 3). Notice how cues from the environment are triggering an inflexible habit, emotionally driving the mind to carry out some chunk of habitual behavior.

Berridge adds that this effect is potent and can last for a long time: "Mesolimbic sensitization renders brain 'wanting' systems hyper-reactive to drug cues and contexts, thus conferring more intense incentive salience on those cues or contexts. Consequently, addicts have stronger cue-triggered urges and intensely 'want' to take drugs. 'Liking', by contrast, need not increase with sensitization, and may even decrease. Sensitized 'wanting' can persist for years, even if the person cognitively doesn't want to take drugs, doesn't expect the drugs to be very pleasant, and even long after withdrawal symptoms have subsided" (p. 5). And it is dependent upon mental networks being triggered by the environment: "Surges of intense 'wanting' are most likely to be triggered when drug cues are encountered (or imagined) in contexts previously associated with taking drugs” (p. 5).

If one wishes to get rid of a habit, one must approach it at the level of mental networks. That is because the mental network decides whether or not a habit will be performed. Graybiel points this out: "Lesion studies show that the medial prefrontal cortical region, called infralimbic (IL) cortex, in rodents, like the DLS, is necessary for habits to be performed... This work is critical to any account of the role of the striatum in habit formation, as it suggests a form of cortical control that can, on a moment-bymoment basis, determine whether a behavior is performed habitually or not” (Graybiel, 2018, p. 10). And this same principle applies in humans to both physical and mental habits. "Habits of thought, at least in humans, are probably as common as motor habits and, like motor habits, are vulnerable to pathologic distortion” (p. 11).

A mental network becomes triggered when it encounters input that resembles its structure, and a mental network will impose its structure upon the mind when triggered. This means that addictive habits are dependent upon the environment: "Early animal studies showed that giving drug in a test environment where it never before was experienced can completely prevent the expression of behavioral and neural sensitization, even when it clearly has been induced, whereas a previously drug-associated context enables the sensitized response to fully reappear again when drug is retaken. That is, sensitized 'wanting' urges are much more likely to occur in drug-associated contexts than in biomedical neuroimaging situations. Recent neuroimaging evidence indicates that drug-related contexts gate 
sensitized brain reactions in humans too” (Berridge, 2016, pp. 7-8). Thus, one can prevent a mental network from being activated by avoiding anything in the environment that will trigger this mental network. Among other things, this means that breaking a habit is often confused with avoiding the context that triggers the habit. A habit that is being avoided remains intact under the surface waiting to be activated by the appropriate environmental triggers.

Addictive habits can be effectively treated by triggering a mental network and then preventing it from expressing itself. This is commonly used for treating OCD: "The final treatment we will consider in the context of habit learning in compulsive disorders is Exposure and Response Prevention (ERP), which works best when used in conjunction with SSRIs in OCD. In ERP, the patient undergoes (i) symptom provocation via exposure to relevant stimuli or situations and (ii) must resist the urge to perform the compulsive act. This treatment does not only produce a reduction in compulsive responding, but also concurrently causes the urge to respond and obsessive thoughts to dissipate. So, for example, if the OCD patient had repetitive thoughts about contamination and compulsively handwashed, the patient would be exposed to dirt or requested to put their hands in toilet water and then prevented from immediately washing their hands" (Gillan, 2016, p. 835). (An SSRI is a selective serotonin reuptake inhibitor, which allows existing serotonin to be more effective. We will see in the next section that serotonin appears to enhance Contributor thought, which would empower goal-oriented behavior, making it easier for a person to choose not to carry out a habit.) Looking at this cognitively, a mental network will eventually fall apart if it is repeatedly triggered and then exposed to content that is incompatible with its structure.

Gillan add that this treatment is effective but unpleasant: "The major problem with both ERP and abstinence is that these therapeutic strategies are not tolerated well by patients” (p. 835). Using current social language, ERP is the opposite of a social 'safe space'. The purpose of ERP is to place a person within an environment that challenges the structure of existing mental networks. In contrast, the purpose of a 'safe space' is to place a person within an environment where existing mental networks are not challenged, but rather reaffirmed.

We have seen that Exhorter thought is needed to change mental networks, and that this emotional shift involves the ventral striatum and dopamine. Dopamine is also required to change contexts at a more local level, and this happens within the dorsal striatum. This can be seen in Parkinsonian patients, who have lost most of the cells of the substantia nigra that send dopamine to the dorsal striatum.

Emotional intensity can provoke freezing of movement in patients with Parkinson's disease (PD). For instance, one experiment showed emotional pictures to Parkinsonian patients and found "that gait initiation was influenced by the emotional valence of the visual stimuli in PD patients with FOG [freezing of gait]. The main findings were longer reaction times and shorter step sizes for forward steps in response to unpleasant images in PD patients with FOG respect to PD patients without FOG and controls. Furthermore, changes in RT [reaction time] performance in response to unpleasant images positively correlated with the 'frequency' of FOG episodes. These findings suggest that emotional load plays a role in gait disturbances of PD patients with FOG” (Lagravinese, 2018, p. 6).

A connection between strong emotions and freezing of movement can also be seen in the normal life of Parkinsonian patients: "MBs [motor blocks] are more frequent and severe when patients with PD are 
exposed to situations producing emotional distress, such as walking in crowded places, speaking to audiences, or signing documents while being watched” (Starkstein, 2015, p. 121). "There was a significant association between the severity of both MBs and festination and emotional distress. Conversely, there was no significant association between MBs or festination and anxiety disorders (generalized anxiety disorder, agoraphobia, social phobia, and panic disorder) or minor or major depression. Second, patients with MBs and emotional distress had more severe MBs and festination, more frequent falls due to freezing... than patients with MBs and no emotional distress” (p. 124). (Festination describes walking in which the steps get faster and smaller in amplitude. The Exhorter person, in contrast, tends to make large, decisive movements.)

Parkinsonian freezing tends to occur when transitioning from one context to another: “The major variables of the first principal component in 14 FOG situations were unfamiliar places, unpredictable schedule changes, entering an automatic door, when another person suddenly crossed, and change in the walking surface... Getting on/off a public transport and crowded places were major variables for the second principal component” (Ishii, 2017,p. 2151). This freezing often happens when moving through a narrow doorway from one physical room to another, and also happens when physically walking while watching on virtual reality goggles a scene portraying walking through a doorway: "Our virtual doorways induced freezing-like episodes in the majority of our PD-f patients. These episodes ranged from $1 \mathrm{~s}$ to $18 \mathrm{~s}$, with a median of around $8 \mathrm{~s}$. All of these participants had more freezing episodes when presented with the narrow doorway compared to the standard doorway. These findings clearly demonstrate how the visual information presented in the virtual environment influences gait in the same way as real doorways" (Gómez-Jordana, 2018, p. 7). The fact that freezing of gait also happens in virtual environments indicates that the deficiency lies with the mental ability to shift from one context to another.

Putting this together, Exhorter thought handles major emotional transitions involving changes to mental networks, and this involves dopamine and the ventral striatum. The mind uses mental networks to represent schemas. There is also an emotionally-driven dopamine circuit within the dorsal striatum that involves changes from one mental schema to another, in which one is not reprogramming schemas, but rather mentally shifting from one schema to another.

This mental shifting involves adjusting mental networks at a more detailed level: "Impaired attentional set-shifting is thought to constitute one of the core cognitive deficits found in Parkinson's disease” (Fallon, 2016, p. 24). “The psychological mechanisms underlying impaired attentional control in PD patients involve an inability to disengage from previously relevant information and to re-engage with previously irrelevant information. The results of this study are consistent with previous reports” (p. 31). Looking at this cognitively, focusing upon what is significant or ignoring what is insignificant is not impaired, because one is simply being guided by existing mental networks. Instead, the dysfunction arises when one has to alter a mental network by either ceasing from being guided by some significant detail or else starting to become guided by some detail that was insignificant. 


\section{Technical Thought versus Mental Networks}

In addiction, Contributor value and goal-oriented behavior become overwhelmed by habits driven by mental networks. The balance can also tip the other way: thinking can focus upon Contributorcontrolled technical thought to the exclusion of mental networks.

Looking at this in more detail, concrete technical thought emerges when Contributor thought places the mind within some limited framework of value and goal-oriented behavior. Technical thought is always guided by the rules of some game, and a game is always played within the boundaries of some playing field. With concrete technical thought, this could be a real game, the 'game' of some business adventure, or a rat running a maze. In a similar manner, abstract technical thought is based in precise definitions and uses some form of logic to pursue a Teacher goal of elegance, beauty, or simplicity. Here too, thinking becomes limited to some restricted set of well-defined terms. Mathematics, computer programming, and logic are prime examples of abstract technical thought, as are most accredited professions.

Mathematics use a different part of the brain than normal linguistics: "In mathematicians, we found essentially no overlap of the math-responsive network with the areas activated by sentence comprehension and general semantic knowledge.” (Amalric, 2016, p. 4915). Instead, “we observed the activation of a restricted and consistent network of brain areas whenever mathematicians engaged in high-level mathematical reflection. This network comprised bilateral intraparietal, inferior temporal, and dorsal prefrontal sites. It was activated by all domains of mathematics tested (analysis, algebra, topology, and geometry) and even, transiently, by meaningless mathematical statements” (p. 4915). Contributor thought combines Perceiver and Server and assembles its well-defined rules from a subset of Perceiver facts and Server sequences. Perceiver thought and Server thought use the parietal and dorsal prefrontal. This explains why mathematical thought emphasizes bilateral intraparietal and dorsal prefrontal sites. (Amalric explains that the inferior temporal region is being activated by the visual symbols that are used in mathematics: "In particular, bilateral ventral inferior temporal areas corresponding to the visual number form area were activated by high-level mathematics as well as by the mere sight of numbers and mathematical formulas” (p. 4915).)

The brain areas used by higher mathematics are also used by other forms of rigorous thought, supporting the idea that higher mathematics should be viewed as a version of abstract technical thought: "The math-responsive circuit that we observed in professional mathematicians also appears to be involved in a broad range of cognitive processes. It activates in a variety of effortful problemsolving tasks akin to IQ tests, as well as in domain-general logical, inferential or relational reasoning” (Amalric, 2017, p. 7).

This means that higher mathematics are different cognitively than the math anxiety that was discussed earlier. Higher mathematics use technical thought guided by the TMN of some paradigm within Teacher thought. Math anxiety, in contrast, occurs when the mind lacks an understanding of mathematics in Teacher thought and approaches mathematics from a personal Mercy perspective.

A focus upon technical thought to the exclusion of mental networks can be seen in autism, and mathematical abilities are correlated with autism: "People with superior mathematical abilities turn out to have an autism spectrum disorder more often than others do. The empathising-systemising theory 
proposes that this link is mediated by these individuals' stronger tendency to systemise (detect patterns, derive rules), along with the fact that mathematics is the perfect example of a rule-based, lawful system” (Bressan, 2018, p. 1). In contrast, it is well-known that autistic people have problems dealing with the mental networks of social interaction: "Anxiety and worry about social situations are common experiences for individuals with autism spectrum disorders (ASD). Although the assessment of social anxiety (SA) can prove complex due to diagnostic overlapping and overshadowing, data obtained from epidemiological and clinical samples indicate that up to $50 \%$ of young people and adults with ASD have clinically significant SA symptoms” (Spain, 2017, p. 35). This contrast was seen previously when comparing TMNs with MMNs.

A dichotomy between technical thought and mental networks can also be seen in gender: "It is a wellknown and widely lamented fact that men outnumber women in a number of fields in STEM, including physics, mathematics, and computer science. The most commonly discussed explanations for the gender gaps are discrimination and socialization, and the most common policy prescriptions are targeted at these ostensible causes" (Stewart-Williams, 2019, p. 2). As the authors point out, it is common today to blame this gender gap upon social discrimination. However, a more careful examination indicates that it is ultimately rooted in the distinction between technical thought and mental networks: "By far the largest sex difference was that for interest in 'people jobs' vs. 'things jobs.' People jobs are jobs that center on interacting with and helping other human beings; things jobs are jobs that center on working with objects, machines, or abstract rules. Members of both sexes can be found at every point on the people vs. things continuum; however, more men than women exhibit a preference for things jobs, whereas more women than men exhibit a preference for people jobs” (pp. 56). Saying this more simply, "men score somewhat higher than women on tests of systemizing, whereas women score somewhat higher than men on tests of empathizing” (p. 17). In other words, males tend to emphasize technical thought while females tend to emphasize mental networks. This is a statistical statement that would be modified by cognitive style. For instance, a female Mercy person would naturally place a greater emphasis upon mental networks than a female Contributor person. And this is not just a social distinction because "The sex difference in interest in things vs. people seems stubbornly resistant to change” (p. 9).

I should add that the female bias towards mental networks is not limited to MMNs of identity and culture, but also includes TMNs of integrated understanding and verbal skill. (This is important to point out because Teacher thought and Teacher emotion are not widely recognized.) Female thought is naturally advantaged at empathy and it is also naturally superior at verbal skills. Quoting further from Stuart-Williams: "More males than females exhibit 'math tilt' (math > verbal), whereas more females than males exhibit 'verbal tilt' (verbal > math)” (pp. 16-17). This distinction emerges very early in life: "The sex difference in mental rotation, for instance, can be detected by three months of age and the sex difference in language ability can be detected by seven months” (p. 20).

The cognitive distinction between male and female thought appears to be partially independent of the physical distinction between male and female gender. That is because "gay men tend to have spatial and linguistic abilities comparable to those of straight women, whereas lesbians tend to have spatial abilities comparable to those of straight men” (p. 22). As Steward-Williams points out, "the near- 
reversal of the usual spatial vs. language pattern is hard to reconcile with the claim that this pattern is due primarily to social forces” (pp. 22-23).

Moving on, I suggest that obsessive-compulsive disorder (OCD) results from inadequate interaction between mental networks and technical thought.

OCD is related to stress: "Individuals with obsessive-compulsive disorder often identify psychosocial stress as a factor that exacerbates their symptoms, and many trace the onset of symptoms to a stressful period of life or a discrete traumatic incident” (Adams, 2018, p. 1). I suggested earlier that Exhorter value within the striosomes overrides Contributor goal-oriented behavior when sufficiently emotional mental networks become involved. This switching of mental gears occurs when one encounters stress or trauma: "Self-report studies indicate that 25-67\% of OCD patients report significant life events (a majority of which are stressful) in relation to the onset of their OCD” (p. 2).

The stress itself does not cause the OCD. Instead, what matters is the way that one responds to this stress. Adams explains that "a similar stressor can produce disparate effects on two individuals, producing pathology in one but minimal effects, or even enhanced resilience, in another” (p. 2). Mental networks become hyperactive in OCD: "Hyperactivity has been reported in the medial frontal cortex (mFC) (especially the anterior cingulate cortex (ACC) and orbitofrontal cortex (OFC)) and the striatum” (p. 3). And the ventral striatum becomes more active in a narrow-minded fixated manner. There is "increased functional connectivity between the ventral striatum and mPFC, but decreased global brain connectivity in the ventral striatum/nucleus accumbens” (p. 3). Finally, there is a shift from goal-oriented behavior to habits: “An overreliance on habitual behaviors has been proposed to be a core mechanism in the development of OCD symptomatology; individuals with OCD exhibit a bias toward inflexible habits, relative to more flexible, goal-directed behavioral control” (p. 4).

Stated succinctly, I suggest that OCD is treating an unwanted mental network as a paradigm for technical thought rather than using Exhorter thought to change the mental network. "Individuals with OCD interpret their intrusions as more meaningful and distressing and, as such, habitually avoid situations or stimuli that may trigger the intrusions, or perform compulsive rituals in attempts to control obsessions and concomitant negative affect. OCD patients typically feel an urge to perform repetitive compulsions, often under strict, idiosyncratic guidelines; these behaviors are often irrational or performed to extreme excess" (p. 1).

The progression is as follows: Instead of addressing the unwanted mental network, it is being mentally suppressed, and environmental situations that might trigger the mental network are being avoided. However, the presence of 'significant details' is causing Exhorter thought to focus upon this unwanted mental network. But when the unwanted mental network becomes activated, Exhorter thought is not being used to change the mental network. Instead, Contributor-controlled technical thought is being used, which is treating the mental network as a goal or paradigm. Instead of learning from the mental network, Exhorter thought is being motivated by it. And when the technical thought fails to eliminate the unwanted mental network, then technical thought is not being abandoned. Instead, it is being carried out more carefully, more precisely, and more repetitively. When technical behavior continues to be repeated, then this will lead to the formation of a TMN that will act as the paradigm for this technical behavior. This TMN will then drive the compulsive rituals and obsessions. 
Looking at this from another perspective, the mental networks in the orbitofrontal cortex that drive OCD are being followed in an isolated manner rather than being connected with other mental networks by the ventromedial prefrontal cortex: "Under certain conditions, a decreased inhibitory influence of the vmPFC on the OFC might likewise underlie excessive OFC activation in patients with OCD (Alves-Pinto, 2019, p. 10).

Saying this another way, OCD patients are deficient at using understanding to change behavior: "While OCD patients (like controls) correctly updated their confidence according to changes in the environment, their actions (unlike those of controls) mostly disregarded this knowledge. Therefore, OCD patients develop an accurate, internal model of the environment but fail to use it to guide behavior" (Vaghi, 2017, p. 348). Thus, rational understanding is being acquired, but this is not affecting the mental networks that drive behavior. Using cognitive language, the Perceiver facts and Server sequences of rational understanding are not known with sufficient confidence to be able to survive emotional pressure from the mental networks driving the OCD.

Another paper on OCD explicitly discusses this idea of placing confidence in knowledge. The authors use the example of predicting where a tennis ball will land: "If you are not confident that your belief is true, you should be very influenced by a surprising serve, while if you are already quite certain about your belief, it is more likely that a surprising outcome is due to chance and therefore you should update your belief less” (Scholl, 2017, p. 250). OCD patients are deficient at using confidence to hold on to their beliefs: "OCD patients did not differ in their explicit confidence ratings from healthy controls. However, they did not adapt their behavioral learning rates in the same way. They made large behavioral adjustments even when their explicit reports had indicated high confidence” (p. 250). Explaining the study more carefully, as the ball in the experiment continued to land in a certain area, the control subjects gained confidence in knowing where the ball would land and they used this knowledge to guide their behavior. In contrast, OCD patients could verbally predict where the ball would land, but this knowledge did not acquire sufficient confidence to guide their behavior.

One can describe this cognitive disconnect using the two concepts of 'myopic value' and 'prospective value' that were mentioned earlier in this essay. The OCD patient is following myopic value in order to carry out the compulsions in a technically proficient manner. Myopic value involves Contributor thought and the dorsal anterior cingulate: "The myopic average value of alternatives and value of the best alternative are reflected in the activity of dACC" (Kolling, 2018, p. 1080). But the OCD patient is not using prospective value to question the value of the mental networks that are driving the obsessivecompulsive behavior. That is because prospective value requires factual input from the dorsolateral prefrontal cortex: "Prospective value, unlike myopic value, was an important determinant of the strength of interactions between dACC and dIPFC” (p. 1080). Consistent with this, electrical stimulation of the dorsolateral prefrontal cortex can reduce obsessive-compulsive behavior (Ghaffari, 2018; Park, 2017). It is not clear from these papers precisely how the dorsolateral prefrontal cortex should be electrically stimulated, but these papers demonstrate that dorsolateral prefrontal stimulation has an effect upon OCD behavior.

Looking at this psychologically, Cognitive Behavior Therapy (CBT) is an effective treatment for OCD. CBT combines Exposure and Response Prevention therapy (ERP) with Cognitive Therapy (CT). We have already seen that ERP challenges the mental networks that are driving unwanted behavior. CT 
examines and questions the implicit beliefs that lie behind these mental networks, helping the dorsolateral prefrontal to bring a factual perspective to the emotional urges.

Going further, preliminary evidence indicates that cognitive behavior therapy may also improve social skills in autistic individuals. "Participants (three adults and one child) were diagnosed with ASD [autism spectrum disorder] and social anxiety disorder. There were commonalities in interventions and techniques used: participants were encouraged to identify and challenge negative thoughts, enter anxiety provoking social situations, and develop new ways of coping. Unlike CBT for SA in non-ASD individuals, treatment also included social skills interventions. Outcomes were assessed using self- or informant-reports. Improvements in social anxiety and depressive symptoms, social skills, and activity levels were noted” (Spain, 2017, p. 34).

\section{Serotonin}

Mental symmetry suggests that dopamine is related to Exhorter, serotonin to Contributor, and noradrenaline to Facilitator. This section will look at serotonin and the next section will look at noradrenaline.

The three composite styles of Exhorter, Contributor, and Facilitator all use information that is generated by the four simple styles of Teacher, Server, Mercy, and Perceiver. Studying personality indicates that the composite styles use the same information as the simple styles but process this information in a different manner. The simple styles emphasize the information itself while the composite styles focus upon using the information to drive thought and behavior. Neuromodulators clarify how this might work. The four simple styles appear to be consciously aware of processing within the cortex. In contrast, the three composite styles use subcortical processing to manipulate cortical information from a distance while using the three monoamines of dopamine, serotonin, and noradrenaline to modify the way that the cortex handles information. (This statement does not attempt to explain consciousness, but rather clarify how it functions, using the working definition that consciousness is related to control and awareness.)

Serotonin is referred to as 5-HT. There are many different serotonin receptors, but the two primary varieties are 5-HT1A and 5-HT2A. These two have quite different effects. A recent paper suggests "that serotonin neurotransmission enhances two distinct adaptive responses to adversity, mediated in large part by its two most prevalent and researched brain receptors: the 5-HT1A and 5-HT2A receptors. We propose that passive coping (i.e. tolerating a source of stress) is mediated by postsynaptic 5-HT1AR signalling and characterised by stress moderation. Conversely, we argue that active coping (i.e. actively addressing a source of stress is mediated by 5-HT2AR signalling and characterised by enhanced plasticity (defined as capacity for change). We propose that 5-HT1AR-mediated stress moderation may be the brain's default response to adversity but that an improved ability to change one's situation and/or relationship to it via 5-HT2AR-mediated plasticity may also be important - and increasingly so as the level of adversity reaches a critical point. We propose that the 5-HT1AR pathway is enhanced by conventional 5-HT reuptake blocking antidepressants such as the selective serotonin reuptake inhibitors (SSRIs), whereas the 5-HT2AR pathway is enhanced by 5-HT2AR-agonist psychedelics" (CarhartHarris, 2017,p. 1091). 
These two responses can both be seen in the Contributor person. We looked at the relationship between Contributor thought and technical thought. Reviewing briefly, technical thought is based upon a limited collection of well-defined Perceiver facts and Server sequences-the 'rules of the game', and technical thought is always limited to some restricted playing field where these rules apply. Technical thought is emotionally guided by the mental network of some Mercy goal or Teacher paradigm, and Contributor thought chooses between the alternatives suggested by Exhorter thought. 5-HT1A enhances the ability of Contributor thought to keep the mind within the confines of the current 'game' without losing control to emotions; it prevents the mind from succumbing to stress. One could refer to this as Contributor confidence - the ability to pursue some plan without losing control to the rest of the mind.

There is also the creative Contributor person who does not limit the mind to rigorous technical thought but rather functions in a manner that is out-of-control, allowing Exhorter thought to come up with ideas that lie 'outside of the box' of the current 'game'. This creative mode of Contributor thought appears to be enhanced by 5-HT2A. Study of personality indicates that the confident Contributor person can perform risky activity without being plagued by doubts or uncertainty, if sufficient contingency planning has been done to ensure that 'all of the bases are covered'. In contrast, the creative Contributor person is prone to various fears and anxieties.

Carhart-Harris describes the function of these two varieties of serotonin in more detail. Looking first at the trait of confidence, if serotonin is reduced, then this gives Exhorter thought freedom to be driven by strong mental networks: "One of the most reliable behavioural effects of reducing 5-HT transmission in the brain is to increase impulsive and aggressive behaviours” (Carhart-Harris, 2017, p. 1094). This aspect of Contributor control is related to 5-HT1A: "There are solid grounds to believe that the antiaggression and impulsivity effects of 5-HT are mediated by postsynaptic 5-HT1A receptor signalling” (p. 1094). Contributor confidence is necessary when one must make choices to avoid unpleasant consequences: "Punishment is one of the most effective means of stimulating 5-HT release. Several studies have demonstrated that anxiety and stress can profoundly increase synaptic 5-HT. Consistent with previous theories, it seems reasonable to suppose that brain 5-HT functions to alleviate psychological distress under adverse conditions - thereby improving coping and resilience” (p. 1096). Saying this more generally, "there is a wealth of evidence that 5-HT is involved in the regulation of mood" (p. 1099).

5-HT2A has quite a different effect. As another author points out, "There is extensive evidence that the 5-HT2A receptor is responsible for the neuropsychological effects of serotonergic hallucinogens in animal models used for experimentation as well as in human subjects” (López-Giménez, 2018, p. 5). "Although hallucinogens do not bind exclusively to 5-HT2A receptors (LSD binds to most 5-HT receptor sub-types as well as to dopaminergic and adrenergic receptors), it has been evidenced in both humans and experimental animals that the activation of 5-HT2A receptors is necessary to generate hallucinogenesis and a related behavioral response in animals” (p. 6).

Carhart-Harris suggests that 5-HT2A puts the mind in a more flexible state: "One may think of 5HT2AR signalling as functioning to induce an entropic state characterised by enhanced flexibility and malleability during which work can be done that, upon cooling, may leave a lasting change” (CarhartHarris, 2017, p. 1103). This makes it possible for the mind to think 'outside of the box': "Reports of 'over-view' type insights, i.e. an improved ability to see the 'bigger picture' under psychedelics, are 
relatively common among user, participant and patient reports and 'aha' type insights have been described” (p. 1103). Saying this another way, “psychedelics are strongly associated with unconventional thinking, vivid imagery and imagination and suggestibility” (p. 1103).

Carhart-Harris points out in a later paper that the cognitive effect of 5-HT2A is highly dependent upon the context: "The main hypothesis of this article is that the therapeutic action of psychedelics is fundamentally reliant on context - both in the psychological and environmental sense. It is argued that neglect of context could render a psychedelic experience not only clinically ineffective but also potentially harmful - accounting, in part, for the negative stigma that still shackles these drugs" (Carhart-Harris, 2018, p. 1). In other words, 5-HT2A has no direct impact upon either mental networks or Exhorter motivation. Instead, it steps back in Contributor thought, giving Exhorter thought freedom to pursue existing mental networks. Thus, 5-HT2A could be compared to a government giving freedom to its citizens. The results will be dependent upon what the citizens want to do. Saying this another way, the impact of laissez-faire capitalism (and Contributor persons tend to be the primary advocates of such a system) will depend heavily upon the cognitive development of the average citizen.

This indirectly increases the level of dopamine: "In the mesolimbic system, 5-HT2AR activation facilitates DA cell activity and DA release” (Howell, 2014, p. 184). “5-HT2AR may also indirectly influence DA release through modulation of excitatory inputs from the PFC. Both systemic and direct administration of DOI into the PFC resulted in increased firing rates and DA release in the VTA" (p. 184). (DOI is another psychedelic drug that enhances brain serotonin). I searched for a paper that connects the activity of 5-HT2A with the striosomes and all I could find was the following phrase in the abstract of an older pay-walled paper: "These results confirm the presence of 5-HT2A receptors in human striosomes” (López-Giménez, 1999, p. 3761). Thus, 5-HT2A could be interpreted as Contributor thought requesting Exhorter thought to focus upon mental networks.

Carhart-Harris seems to be advocating the use of LSD. Similarly, a 2019 article in Scientific American reports micro-dosing on LSD as “the 'productivity hack' popular among Silicon Valley engineers and business leaders. Microdosers take regular small doses of LSD or magic mushrooms. At these doses, they don't experience mind-bending, hallucinatory trips, but they say they get a jolt in creativity and focus that can elevate work performance, help relationships, and generally improve a stressful and

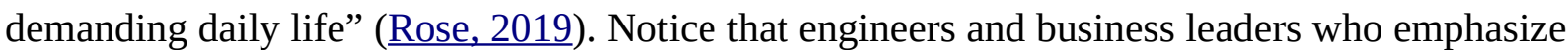
technical thought are micro-dosing LSD to help them come up with more creative solutions, consistent with the suggestion that 5-HT2A allows Contributor thought to move beyond confidence to creativity.

Mental symmetry suggests that micro-dosing LSD is dealing with the symptom rather than addressing the cause. The fundamental problem is that Western scientific thought emphasizes technical thought in a specialized, objective manner which ignores Teacher emotions and suppresses Mercy feelings. The solution is a form of cognitive reappraisal in which one reprograms mental networks to be compatible with rational thought. This will naturally cause a person to become more creative and emotionally open. Micro-dosing LSD artificially encourages technical thinkers to use technical thought in a more open-minded manner.

We have looked at Contributor confidence and Contributor creativity. The Contributor person can also function in a rigid manner which could be described as Contributor control. This is under the control of 
the subthalamus, which is an aspect of the indirect path through the basal ganglia. The subthalamus carries out two related functions, which are "first that the subthalamic nucleus plays a role in adjusting response thresholds and speed-accuracy trade-offs and second that it is involved in reactive and proactive inhibition and conflict resolution” (Obeso, 2014, p. 1).

Obeso explains that the second role can be stated more simply as a 'no go' or 'hold your horses' signal which is controlled by what is referred to as the hyperdirect pathway: "The hyperdirect pathway connects the cortex with the subthalamic nucleus (STN) providing the quickest route for the cortex to influence the inhibitory output from the basal ganglia and to, in turn, regulate and fine-tune cortical activity” (p. 2). The term 'hyperdirect' refers to the speed of this pathway; it could be compared to a hotline through which the cortex can call up the basic ganglia and order 'hold your horses'. The hyperdirect pathway is actually an aspect of the indirect pathway: "subthalamotomy disrupts the hyperdirect and indirect, but not the direct pathway” (p. 10).

The first role involves a trade-off between speed and accuracy. One treatment for Parkinson's disease is to damage the subthalamus. This is known as a subthalamotomy. Obeso reports that "Patients with right subthalamotomy had significantly faster 'critical' Go reaction times with their contralesional hand than unoperated patients and did not differ from controls, suggesting that their speed of response initiation was 'normalized' to the level of controls. However, accuracy was compromised as patients with either right or left-sided subthalamotomy made significantly more discrimination errors than unoperated patients and controls. This profile of enhanced speed coupled with greater discrimination errors suggests subthalamotomy had an impact on speed-accuracy tradeoffs” (p. 7).

The subthalamus is also related to speech fluency, consistent with the idea that the basal ganglia are also involved in abstract thought (Lipski, 2018). The precise role carried out by the subthalamus in speech is still uncertain and the effect of DBS (deep brain stimulation) depends upon the frequency at which the subthalamus is electrically stimulated (Grover, 2019).

Summarizing, the subthalamus makes it possible to function in a manner that is slower but also more cautious. This is an important function which makes it possible to vary one's behavior between emphasizing speed or accuracy. Study of personality indicates that some Contributor persons habitually function in a manner that is slow, deliberate, and pedantic, which could be compared to driving a car with one's foot continually on the brake.

\section{Thalamus}

The three composite styles of Exhorter, Contributor, and Facilitator could be viewed as a 'three stage mental pump' that drives the mind. The third stage is provided by Facilitator thought, and this appears to involve the thalamus. Information from the basal ganglia, where Exhorter and Contributor processing occur, passes through the thalamus on its way back to the cortex. In addition, the thalamus sends and receives information from most of the cortex. Finally, four of the five senses (but not smell) pass through the thalamus on their way to the cortex. (Odor is an effective way of triggering a mental network, because the sense of smell passes directly to the orbitofrontal cortex without going through the thalamus.) 
The Facilitator person observes both thought and sensory information from a distance, aware of everything within the current context at a superficial level. The Facilitator person mixes and adjusts these various streams of thought and sensation, similar to the mixing board in a sound studio.

The thalamus plays such a role of 'facilitating' the cortex: “The thalamus regulates functional connectivity within and between cortical regions, determining how a cognitive process is implemented across distributed cortical microcircuits. Within this framework, thalamic circuits do not necessarily determine the categorical content of a cognitive process (e.g., sensory details in feature-based attention), but rather provide a route by which task-relevant cortical representations are sustained and coordinated" (Nakajima, 2017, p. 127). Notice that the thalamus is not processing information, but rather balancing and coordinating the way that the cortex processes information. In a similar manner, Facilitator thought mixes and balances processing that is happening within other cognitive modules.

The Facilitator person is often connecting one person with another, and is naturally talented at managing people and projects. Likewise, Nakajima says that "this newly recognized thalamic role, as a master regulator of functional cortical connectivity, places this subcortical structure closer to the center of cognitive control” (p. 127).

Facilitator thought becomes prominent in a group or society when one must mediate between many different viewpoints. A similar role can be seen in the medial dorsal thalamus (MD), the part of the thalamus that connects with the frontal lobes: "Current evidence suggests the MD is particularly important during rapid trial-by-trial associative learning and decision-making paradigms that involve multiple cognitive processes” (Mitchell, 2015, p. 76). “The cognitive deficits shown in animals with loss of MD, and specifically MDmc in primates, seem to be associated with tasks that require information from multiple cognitive processes to be linked together for successful, optimal performance” (p. 82).

Facilitator thought has a wide awareness, but this awareness is limited to the current context. Thus, Facilitator thought notices when something does not fit into the current context. This can be seen in another part of thalamus, known as CM-Pf (central median and parafascicular): "CM-Pf neurons are strongly activated at sudden changes in behavioral context, such as switches in action-outcome contingency or sequence of behavioral requirements, suggesting that their activity may represent change of context operationalized as associability” (Yamanaka, 2018, p. 1).

Exhorter thought notices significant details. Facilitator thought, in contrast, recognizes the unexpected, whether it is significant or not: "The activity of CM-Pf neurons, however, is different from that of dopamine neurons, because it is similar whether or not the unexpected stimuli are associated with reward... The CM-Pf receives information about environmental events coming through multimodal sensory channels, is sensitive to unexpectedness and surprise, and has no specific sensitivity to reward" (p. 4).

Facilitator thought also recognizes unexpected changes in rules or social norms: "Neurons in the CMPf are strongly activated at changes in behavioral situations such as altered action-outcome contingencies and transitions in behavioral requirements, suggesting the encoding of the associability of behavioral context, an average of prediction errors generated across the past few trials” (p. 11). The Facilitator person is often attracted to a bureaucracy, in which rules are followed in a consistent 
manner, regardless of whether these rules are significant or not. Similarly, the Facilitator person naturally becomes an enforcer of social norms. Another paper relates the function of the parafascicular thalamus more explicitly to rules within some context: "Input from a part of the thalamus, the parafascicular nucleus, onto cholinergic interneurons in the posterior compartment of the dorsomedial striatum (pDMS), is critical to the appropriate creation of new states during learning. Note that we use 'state' here to refer to a high order representation of the environment in which actions are being chosen” (Schoenbaum, 2013, p. 3). The authors use the illustration of moving to Australia, where one drives on the opposite side of the road. "Optimally, rather than overwriting your original strategy for crossing the street, upon experiencing the strange driving habits in your new hometown, you would form a new 'state' of 'I am in Sydney' and learn new mappings from actions to goals... relevant to that state” (p. 3).

Moving further, the mixing and adjusting performed by Facilitator thought involves an interplay between the thalamus, noradrenaline, and the locus coeruleus (LC), which is the primary source of brain noradrenaline. (NA is short for noradrenaline, which is also referred to as norepinephrine, or NE). "Through direct activation of the LC-NE system, we found that elevated LC-NE activity causes a dramatic increase in thalamic feature selectivity and improvement in information transmission. Our results strongly suggest that LC-activation-induced improvement of thalamocortical information transmission is primarily mediated by NE regulation of intrathalamic circuit dynamics via the direct action of NE on $\alpha$-adrenergic receptors in both the VPm and TRN. LC activation improved thalamic information transmission in both anesthetized and awake animals, suggesting LC-NE improvement of thalamic information transmission is a general phenomenon” (Rodenkirch, 2019, p. 11). (TRN is the reticular thalamus, while VPm refers to the ventral posteromedial nucleus of the thalamus, which encodes whisker movement in the rodent.)

The functioning of the various parts of the thalamus is itself adjusted and balanced by the thalamic reticular nucleus (TRN): "The TRN forms a thin shell of neurons that covers the lateral and anterior surface of the dorsal thalamus, and it receives input from branches of both thalamo-cortical and corticothalamic fibers. The TRN in turn sends its output exclusively to the thalamus and is positioned to provide inhibitory control over thalamo-cortical transmission” (Saalmann, 2012, p. 209). "The TRN has been implicated in playing an important role in selective attention by regulating thalamo-cortical information transmission” (p. 217). Notice that the TRN controls the cortex indirectly through the thalamus, consistent with the behavior of the Facilitator person, who also controls the functioning of the rest of the mind from a distance.

Facilitator thought does its mixing and adjusting in the light of Perceiver facts and Server sequences that remain fixed. On the one hand, the Facilitator person feels muddled when there are no fixed reference points. On the other hand, the Facilitator person feels restricted when mixing and adjusting is not permitted. Looking at this more generally, when there is societal chaos, then Facilitator persons call for structure; when there is rigidity, then Facilitator persons call for freedom.

Facilitator persons may acquire their reference points directly from Perceiver and Server thought or may determine them in a statistical matter by averaging the opinions of emotionally significant people and theories. (This has societal implications. For instance, 'post-truth' was named word of the year in 2016 by the Oxford Dictionary. The behavior of a Facilitator person is quite different in a society of 
'truth' than in one of 'post-truth' because mixing and adjusting will be guided by a different kind of fixed reference points.) Consistent with this, a study of the reticular thalamic nucleus in the monkey found that "some prefrontal cortices have widespread projections that extend beyond the frontal sector of TRN to sites innervated by sensory and motor cortices. This unique type of projection is found for dorsolateral prefrontal cortex (DLPFC) and posterior orbitofrontal cortex (pOFC), and their associated mediodorsal thalamic nucleus (MD), which have a key role in cognition, emotion and memory. Through these projections, the DLPFC and pOFC may control the passage of signals through the thalamus to shift attention to relevant stimuli and suppress distracters” (Zikopoulos, 2012, p. 5338). Thus, the functioning of the thalamic reticular nucleus can be guided either by facts and sequences from the dorsolateral prefrontal, or by mental networks representing people and paradigms within the orbitofrontal cortex.

Going further, Zikopolous discovered a "pathway that may control emotional attention through a projection from the amygdala, the brain's key emotional center, to the inhibitory TRN, a central node in the brain's attentional system. The amygdalar pathway, which had not been described previously in any species, converged at the TRN sites that receive widespread projections from orbitofrontal cortex, which processes the value of stimuli, and from the MD, the principal prefrontal thalamic nucleus” (p. 5346). The Facilitator person can be quite sensual, focusing upon emotional sensations. But Facilitator sensuality is different than the 'party animal' kind of hedonism exhibited by the immature Exhorter person. Instead, it exhibits itself as a kind of philosophy in which sensuality is viewed as a source of truth and meaning.

We saw earlier that Contributor thought is guided by value within the anterior cingulate. In contrast, the Facilitator person often uses experimentation, in which random changes are made and the results are evaluated. For instance, Thomas Edison was a Facilitator person. When developing the light bulb, Edison "tested the carbonized filaments of every plant imaginable, including baywood, boxwood, hickory, cedar, flax, and bamboo. He even contacted biologists who sent him plant fibers from places in the tropics. Edison acknowledged that the work was tedious and very demanding, especially on his workers helping with the experiments” (Franklin Institute, 2019). This switch from goal-oriented behavior to random experimentation is governed by noradrenaline from the locus coeruleus. Rats "can switch to a 'stochastic' mode when challenged with a competitor that they cannot defeat by counterprediction. In this mode, outcomes associated with an animal's actions are ignored, and normal engagement of anterior cingulate cortex (ACC) is suppressed. Using circuit perturbations in transgenic rats, we demonstrate that switching between strategic and stochastic behavioral modes is controlled by locus coeruleus input into ACC” (Tervo, 2014, p. 21). Tervo adds that this same kind of transition occurs within the human mind: "Studies measuring pupillary responses in humans-a known consequence of LC activation-suggest that levels of noradrenergic signaling reflect the degree of uncertainty about the accuracy of one's internal model, with high levels associated with the discarding of an unreliable model and low levels linked to stabilization of an accurate model” (p. 29).

We also saw earlier that the frontopolar cortex uses patterns and analogies to build connections between different contexts. Exploration can be guided by a meta-theory within the frontopolar cortex. For instance, this describes the approach taken by mental symmetry. If the right frontopolar cortex is electrically suppressed, then random exploration will take over: "We used continuous theta-burst TMS 
to selectively inhibit right frontopolar cortex (RFPC) in participants performing the 'Horizon Task', an explore-exploit task specifically designed to separate directed and random exploration. Using this task we find evidence that inhibition of RFPC selectively inhibits directed exploration while leaving random exploration intact” (Zajkowski, 2017, p. 2).

Exhorter thought provides motivation to reach some goal. Facilitator thought, in contrast, makes the best of the existing situation, optimizing within the current context. This distinction can be seen with dopamine and noradrenaline: "Dopaminergic neurons compute outcome value by combining reward and effort-cost information, whereas the activity of noradrenergic neurons increase when monkeys mobilize resources to meet a physical challenge and overcome a difficulty” (Varazzani, 2015, p. 7866). "Monkeys decided to forgo trials requiring high effort costs and small rewards. Our neuronal data indicate a specific implication of SNc neurons in coding such value-based decision making. Conversely, when monkeys committed to perform the trial, they faced the imposed difficulty by producing the required amount of force in conjunction with an activation of both LC neurons and the autonomic system. This suggests a specific implication of NA neurons in mobilizing resources to energize behavior and face the challenge at hand” (p. 7873). (SNc is substantia nigra pars compacta, which is the source of dopamine for the caudate and putamen.)

We saw earlier that emotional stress causes the mind to emphasize Exhorter thought, and that the Exhorter person naturally thrives in situations that are emotionally stressful. The Facilitator person, in contrast, tends to feel muddled in situations of emotional stress. However, the Facilitator person who is in a physical crisis finds that, instead of feeling confused, thinking clarifies and decisions become obvious. This suggests that a distinction needs to be made between psychological stress and physiological stress. Psychological stress enables Exhorter thought, while physiological stress enhances Facilitator thought. Most neurological papers on stress do not make this distinction. However, there is neurological evidence for such a distinction.

We also saw earlier that the emotionally-driven Exhorter-controlled mechanism for shifting contexts is damaged in Parkinson's disease. However, a paralyzed Parkinsonian patient can temporarily reacquire the ability to move in a physical crisis. This is known as paradoxical kinesia: "Although slowness of movement is a typical feature of Parkinson's disease (PD), it has been suggested that severely disabled patients remained able to produce normal motor responses under particular circumstances. For instance, we have all heard the stories of these wheelchair-confined patients who, upon hearing someone shout 'fire' in a building, run out as rapidly as all the other nonparkinsonian occupants, only to 'freeze' again once in a safe area. During the past decades, such anecdotal reports of normal motor behavior in PD patients have been echoed by more controlled experimental findings” (Ballanger, 2006, p. 1491). Saying this another way, we saw earlier that psychological stress leads to freezing in a Parkinsonian patient. In contrast, we see here that physiological stress can temporarily re-enable freedom of movement in a Parkinsonian patient. This shows that these two kinds of stress need to be distinguished from one another because they are enabling different brain circuits.

The mental clarifying that happens to Facilitator thought in a physical crisis is related to noradrenaline coming from the locus coeruleus: "Our results provide the first empirical support for the idea that a momentary increase in arousal engages the LC [locus coeruleus] to selectively process and store prioritized information regardless of its intrinsic emotionality. Our behavioral results revealed that 
threat-induced arousal amplified the effects of top-down priority such that memory for goal-relevant stimuli was selectively enhanced” (Clewett, 2018, p. 1570). Saying this more generally, Facilitator clarity that is enabled by a physical crisis is different than Exhorter-guided emotional reprogramming that is enabled by an emotional crisis: "The notion that our pupil and LC activity results reflect increased attentional modulation rather than emotional arousal, per se, aligns with extant neurobiological models of emotional memory. Specifically, converging theoretical and empirical work demonstrate that the immediate benefit of attention on emotional memory are dissociable from longerterm emotional memory enhancements” (p. 1571).

Looking at physiological stress more carefully, a stressful event will lead to the release of noradrenaline, which will cause a shift in mental emphasis: "Noradrenaline, released within seconds after a stressful event from noradrenergic brain stem nuclei and the adrenal medulla, triggers a reorientation of large scale networks toward the processing of salient stimuli, at the expense of executive control processes" (Wirz, 2017, p. 10). The mental clarifying that is triggered by a stressful event will cause habits to be emphasized over learning: "Stressful events promote, mediated through the actions of noradrenaline and glucocorticoids, a shift from flexible 'cognitive' toward more rigid 'habit' behavior" (p. 10). Wirz explains that such clarity is useful in a physical crisis: "This shift may be highly adaptive, as it helps the organism to save cognitive resources and avoid hesitations or delays during threatening situations. Indeed, there is direct evidence showing that the shift toward habitual learning is beneficial for performance under stress, whereas cognitive learning in the face of stress impairs performance” (p. 14). Wirz does not distinguish explicitly between physiological stress and psychological stress, but it is clear from the description that physiological stress is being described.

A physical crisis will emphasize mental habits over learning. Therefore, one can prepare for an emergency by acquiring the appropriate habits beforehand. This explains why soldiers and emergency responders train critical actions until they can be performed habitually. Looking at this more generally, we saw earlier that noradrenaline encourages random behavior, while we have just seen that noradrenaline also leads to clarity of mind. These two effects appear at first glance to contradict one another, but the difference may lie in the level of training. If training is insufficient, then physiological stress will tend to provoke random behavior. In contrast, if habits have been developed sufficiently through training, then physiological stress may lead to mental clarity instead. I cannot point to any studies to back up this suggestion, except to note that soldiers have historically adopted the random strategy of scattering and running when faced with excessive physiological stress, and that the historical antidote has been to develop rigid habits in soldiers through over-training.

Going further, military training may lead to an increased ability to handle physiological stress, but this can be accompanied by a reduced capacity to handle psychological stress. One paper reports that military training emphasizes noradrenaline produced by the locus coeruleus, and explains that this training may make a person vulnerable to outbursts of anger if mental networks within the ventromedial prefrontal cortex are inadequate: “Angry outbursts are an important feature of various stress-related disorders, and commonly lead to aggression towards other people. Findings regarding interpersonal anger have linked the ventromedial prefrontal cortex (vmPFC) to anger regulation and the locus coeruleus (LC) to aggression” (Gilam, 2017, p. 1561). 
"Soldiers showed an increase in stress symptoms following combat training while civilians showed no such change following civil service... Higher post-combat training stress symptoms were associated with lower pre-combat training vmPFC activation and with higher activation increase in the LC between pre- and post-combat training... Results suggest that during anger-provoking social interactions, flawed vmPFC functionality may serve as a causal risk factor for the development of stress symptoms, and heightened reactivity of the LC possibly reflects a consequence of stress-inducing combat training” (p. 1561).

A recent $\mathrm{PhD}$ thesis mentions the distinction between physiological and psychological stress. The author refers only to dopamine without mentioning noradrenaline, but the statements are consistent with what is being suggested in this essay. "Physiological (i.e. pain, metabolic) stressors are consistently associated with increased dorsal striatal DAergic activity; the ventral striatal DAergic response is less robust. This contrasts with psychological stress, which does not consistently increase striatal DAergic activity in HV [healthy volunteers] but seems to increase DAergic activity in the mPFC, an observation reported in three independent samples of healthy participants” (Vaessen, 2018, p. 106). Vaessen says that physiological stress leads to increased dopamine in the dorsal striatum. Presumably, Facilitator thought is taking charge, it is focusing upon clarity and habits, and Exhorter thought is following. Similarly, Wirz looked at physiological stress and noted that it causes habits to be emphasized within the dorsal striatum, driven by the emotions of the moment from the amygdala enhanced by noradrenaline: "Stress resulted in increased amygdala-dorsal striatum and reduced amygdala-hippocampus connectivity... these findings point to a critical role of noradrenergic arousal in the amygdala for the stress-induced shift toward habit learning” (Wirz, 2017, p. 11). And we saw in the quote from Gilam that military training can lead to outbursts of anger, which we saw earlier is triggered by the amygdala.

Going further, Vaessen relates physiological stress to a physical crisis from the environment, while relating psychological stress to expectations of people represented by mental networks: "Whereas the dorsal striatal DA response seems to be associated with the sensory and affective properties related to the stressor itself, DAergic activity in the ventral striatum varies with subjective expectations about the stressor” (Vaessen, 2018, p. 109). And psychological stress is more prevalent when mental networks representing parents are weak: "In individuals who reported low maternal bonding, and are assumed to be at risk for a broad range of psychopathology, psychological stress increased DAergic activity in the ventral striatum” (p. 111).

In conclusion, personality indicates and neurological data suggest that one should distinguish between physiological and psychological stress. Physiological stress is driven by Facilitator thought and noradrenaline, and will emphasize mental clarity and habits. Psychological stress is driven by Exhorter thought and dopamine, and will emphasize changing or reprogramming mental networks. Part of the confusion between these two kinds of stress may be the result of inadequate cognitive development. It is easy to treat psychological stress as if it is a physiological crisis. One has to learn that 'sticks and stones will hurt my bones, but words will never hurt me'. Similarly, one has to learn that going through a physical crisis does not automatically imply that one needs to abandon core mental networks—as shown by the tendency for trained soldiers with weak mental networks to lose their tempers. 


\section{Conclusion}

A cognitive model that began with a system of cognitive styles and acquired its initial data from an analysis of historical biographies has been mapped onto the functioning of different brain regions. This mapping is both detailed and comprehensive. All seven of the cognitive styles have been included and all the brain regions related to higher thought have been discussed. As far as I know, this type of mapping has not been done before.

Mapping a cognitive model onto neurology risks falling into one of two extremes. On the one side, if not enough neurological detail is given, then the cognitive model may be rejected as non-rigorous. On the other hand, if too much neurological detail is given - especially if one quotes directly from neurological papers, then the paper may read as a summary of neurology, rather than as a mapping of some cognitive model. I am not sure how to address this problem, except to point out that neurology is currently coming up with many details of personality which were first described by the theory of mental symmetry. This assertion can be checked by looking at an early version of the mentalsymmetry.com website dating from 2002. That early version describes the seven cognitive styles using quotes from biographies collated by Lane Friesen, and can be accessed via the wayback machine. In contrast, almost all of the references quoted in this paper date from after 2002. (I should point out that both our understanding of neurology and the state of neurology were significantly more primitive in 2002.)

The theory of mental symmetry contains many details, and this paper has discussed a range of topics. I suggest that the following points are the most significant:

- The existence of Teacher emotion.

- The existence of cognitive styles.

- Mental networks and the distinction between a MMN and TMN.

- The division of thought into mental networks, normal thought, and technical thought.

- A realization that minds are studying minds in neurology and psychology.

If I had to choose one single point from this entire paper, it would be the existence of Teacher thought and Teacher emotion. This understanding is currently lacking. I have found that even those who teach the same system of cognitive styles used by mental symmetry and who talk about 'Teacher persons' confuse Teacher thought with abstract technical thought. However, we have seen in this paper that an understanding of Teacher thought and Teacher emotion can bring clarity to a number of topics.

Another significant concept is that of the mental network. The idea of a schema is quite common and can be found in many forms in various contexts. What appears to be new is the suggestion that a schema uses emotional pressure to impose its structure when triggered, as well as the idea that attempting to dismantle a mental network will provoke a strong emotional backlash. Similarly, paradigms and paradigm shifts have been discussed extensively, but what appears to be new is the idea that technical thought is being implicitly motivated at an emotional level by the TMN of a paradigm. In other words, fictional characters such as Spock or Data of Star Trek who function at a purely logical 
level could not exist, because their supposedly cold, logical reasoning is actually being emotionally driven by the TMN of some paradigm.

Going further, scientific research places a great emphasis upon technical thought. However, when one observes the human brain, then what one finds at the highest level of human cognition is not technical thought, but rather mental networks within the orbitofrontal and medial frontal regions, as well as the analogies and patterns of normal thought within the frontopolar cortex. Thus, minds that emphasize technical thought are attempting to understand minds that are driven primarily by mental networks and normal thought, and not by technical thought.

Academic papers are normally expected to go through the process of peer review. Peer review assumes that one is working within a community of researchers who are using technical thought guided by a common paradigm, and peer review excels at ensuring that one is dealing with accurate data. However, peer review tends to fail when introducing a new paradigm. That is because a new paradigm looks at the same data from a different perspective. In the words of Thomas Kuhn, "One perceptive historian, viewing a classic case of a science's reorientation by paradigm change, recently described it as 'picking up the other end of the stick,' a process that involves 'handling the same bundle of data as before, but placing them in a new system of relations with one another by giving them a different framework"” (Kuhn, 1970, p. 85). The difficulties inherent in a paradigm shift become even greater when one is suggesting for the first time within some field that it is possible to use a paradigm.

Kuhn observes that Teacher emotions of order-within-complexity can play a role in encouraging a person to adopt a new paradigm: "Fortunately, there is also another sort of consideration that can lead scientists to reject an old paradigm in favor of a new. These are the arguments, rarely made entirely explicit, that appeal to the individual's sense of the appropriate or the aesthetic - the new theory is said to be 'neater,' 'more suitable,' or 'simpler' than the old” (Kuhn, 1970, p. 155). Using the language of this paper, one is using a form of cognitive reappraisal guided by Teacher thought and Teacher emotion.

Kuhn adds that "Probably such arguments are less effective in the sciences than in mathematics" (pp. 155-156). Nevertheless, this paper will conclude by pointing out a curious parallel between the structure of science and the thesis of this paper which Teacher thought should find emotionally appealing.

One of the deep mysteries of science is that one can make progress either by searching for empirical data or by working with mathematics at a theoretical level. Paul Dirac, the famous physicist, described this parallel in a lecture given in 1939: "The physicist, in his study of natural phenomena, has two methods of making progress: (1) the method of experiment and observation, and (2) the method of mathematical reasoning. The former is just the collection of selected data; the latter enables one to infer results about experiments that have not been performed. There is no logical reason why the second method should be possible at all, but one has found in practice that it does work and meets with reasonable success. This must be ascribed to some mathematical quality in Nature, a quality which the casual observer of Nature would not suspect, but which nevertheless plays an important role in Nature's scheme” (Dirac, 1940, p. 1).

This paper has shown that a similar relationship exists when studying the brain. One can make progress either by searching for empirical, neurological data or by thinking about cognition at a theoretical level. 
This does not mean that one can use any system of symbolic logic to analyze the physical universe, or that one can use any cognitive model to analyze the brain. But in both cases, if one uses the right kind of theoretical structure in abstract thought, then one finds that one can use either a bottom-up approach or a top-down approach, and the results will converge. As Dirac pointed out, 'There is no logical reason why the second method should be possible at all, but one has found in practice that it does work and meets with reasonable success'. 


\section{References}

Adams, T. G., Kelmendi, B., Brake, C. A., Gruner, P., Badour, C. L., \& Pittenger, C. (2018). The Role of Stress in the Pathogenesis and Maintenance of Obsessive-Compulsive Disorder. Chronic Stress, 2, 247054701875804. doi: 10.1177/2470547018758043

Alves-Pinto, A., Rus, O. G., Reess, T. J., Wohlschläger, A., Wagner, G., Berberich, G., \& Koch, K. (2019). Altered reward-related effective connectivity in obsessive-compulsive disorder: an fMRI study. Journal of Psychiatry and Neuroscience, 44(6), 395-406. doi: 10.1503/jpn.180195

Amalric, M., \& Dehaene, S. (2016). Origins of the brain networks for advanced mathematics in expert mathematicians. Proceedings of the National Academy of Sciences, 113(18), 4909-4917. doi: 10.1073/pnas.1603205113

Amalric, M., \& Dehaene, S. (2018). Cortical circuits for mathematical knowledge: evidence for a major subdivision within the brains semantic networks. Philosophical Transactions of the Royal Society B: Biological Sciences, 373(1740), 20160515. doi: 10.1098/rstb.2016.0515

Ambridge, B., Pine, J. M., Rowland, C. F., Chang, F., \& Bidgood, A. (2012). The retreat from overgeneralization in child language acquisition: word learning, morphology, and verb argument structure. Wiley Interdisciplinary Reviews: Cognitive Science, 4(1), 47-62. doi: 10.1002/wcs.1207

Amemori, S., Amemori, K.-I., Yoshida, T., Papageorgiou, G. K., Xu, R., Shimazu, H., ... Graybiel, A. M. (2019). Microstimulation of primate neocortex targeting striosomes induces negative decisionmaking. doi: 10.1101/668194

Araujo, H. F., Kaplan, J., Damasio, H., \& Damasio, A. (2015). Neural correlates of different self domains. Brain and Behavior, 5(12). doi: 10.1002/brb3.409

Badre, D., \& Nee, D. E. (2018). Frontal Cortex and the Hierarchical Control of Behavior. Trends in Cognitive Sciences, 22(2), 170-188. doi: 10.1016/j.tics.2017.11.005

Baldassano, C., Hasson, U., \& Norman, K. A. (2018). Representation of Real-World Event Schemas during Narrative Perception. The Journal of Neuroscience, 38(45), 9689-9699. doi: 10.1523/jneurosci.0251-18.2018

Ballanger, B., Thobois, S., Baraduc, P., Turner, R. S., Broussolle, E., \& Desmurget, M. (2006). "Paradoxical Kinesis" is not a Hallmark of Parkinsons disease but a general property of the motor system. Movement Disorders, 21(9), 1490-1495. doi: 10.1002/mds.20987

Barrett, J. L., \& Lanman, J. A. (2008). The science of religious beliefs. Religion, 38(2), 109-124. doi: 10.1016/j.religion.2008.01.007

Berke, J. D. (2018). What does dopamine mean? Nature Neuroscience, 21(6), 787-793. doi: 10.1038/s41593-018-0152-y

Berridge, K. C., \& Robinson, T. E. (2016). Liking, wanting, and the incentive-sensitization theory of addiction. American Psychologist, 71(8), 670-679. doi: 10.1037/amp0000059 
Blair, C. (2016). Developmental Science and Executive Function. Current Directions in Psychological Science, 25(1), 3-7. doi: 10.1177/0963721415622634

Bloem, B., Huda, R., Sur, M., \& Graybiel, A. M. (2017). Two-Photon Imaging of Striatum Demonstrates Distinct Functions for Striosomes and Matrix in Reinforcement Learning. doi: 10.1101/199497

Bressan, P. (2018). Systemisers are better at maths. Scientific Reports, 8(1). doi: 10.1038/s41598-01830013-8

Bressler, S. L., \& Menon, V. (2010). Large-scale brain networks in cognition: emerging methods and principles. Trends in Cognitive Sciences, 14(6), 277-290. doi: 10.1016/j.tics.2010.04.004

Brunec, I. K., Robin, J., Patai, E. Z., Ozubko, J. D., Javadi, A.-H., Barense, M. D., .. Moscovitch, M. (2019). Cognitive mapping style relates to posterior-anterior hippocampal volume ratio. Hippocampus, 29(8), 748-754. doi: 10.1002/hipo.23072

Buhle, J. T., Silvers, J. A., Wager, T. D., Lopez, R., Onyemekwu, C., Kober, H., ... Ochsner, K. N. (2013). Cognitive Reappraisal of Emotion: A Meta-Analysis of Human Neuroimaging Studies. Cerebral Cortex, 24(11), 2981-2990. doi: 10.1093/cercor/bht154

Bzdok, D., Schilbach, L., Vogeley, K., Schneider, K., Laird, A. R., Langner, R., \& Eickhoff, S. B. (2012). Parsing the neural correlates of moral cognition: ALE meta-analysis on morality, theory of mind, and empathy. Brain Structure and Function, 217(4), 783-796. doi: 10.1007/s00429-012-0380-y

Bzdok, D., Langner, R., Schilbach, L., Engemann, D. A., Laird, A. R., Fox, P. T., \& Eickhoff, S. B. (2013). Segregation of the human medial prefrontal cortex in social cognition. Frontiers in Human Neuroscience, 7. doi: 10.3389/fnhum.2013.00232

Callaghan, B. L., \& Tottenham, N. (2015). The Neuro-Environmental Loop of Plasticity: A CrossSpecies Analysis of Parental Effects on Emotion Circuitry Development Following Typical and Adverse Caregiving. Neuropsychopharmacology, 41(1), 163-176. doi: 10.1038/npp.2015.204

Carhart-Harris, R., \& Nutt, D. (2017). Serotonin and brain function: a tale of two receptors. Journal of Psychopharmacology, 31(9), 1091-1120. doi: 10.1177/0269881117725915

Carhart-Harris, R. L., Roseman, L., Haijen, E., Erritzoe, D., Watts, R., Branchi, I., \& Kaelen, M. (2018). Psychedelics and the essential importance of context. Journal of Psychopharmacology, 32(7), 725-731. doi: 10.1177/0269881118754710

Caruana, F., Gerbella, M., Avanzini, P., Gozzo, F., Pelliccia, V., Mai, R., ... Rizzolatti, G. (2018). Motor and emotional behaviours elicited by electrical stimulation of the human cingulate cortex. Brain, 141(10), 3035-3051. doi: 10.1093/brain/awy219

Celeghin, A., Diano, M., Bagnis, A., Viola, M., \& Tamietto, M. (2017). Basic Emotions in Human Neuroscience: Neuroimaging and Beyond. Frontiers in Psychology, 8. doi: 10.3389/fpsyg.2017.01432

Chanales, A. J., Oza, A., Favila, S. E., \& Kuhl, B. A. (2017). Overlap among Spatial Memories Triggers Repulsion of Hippocampal Representations. Current Biology, 27(15). doi:

10.1016/j.cub.2017.06.057 
Cheung, V. K. M., Meyer, L., Friederici, A. D., \& Koelsch, S. (2018). The right inferior frontal gyrus processes nested non-local dependencies in music. Scientific Reports, 8(1). doi: 10.1038/s41598-01822144-9

Christoff, K. \& Gabrieli, J.D.E. (2000). The frontopolar cortex and human cognition: Evidence for a rostrocaudal hierarchical organization within the human prefrontal cortex. Psychobiology (2000) 28: 168. https://doi.org/10.3758/BF03331976

Clewett, D. V., Huang, R., Velasco, R., Lee, T.-H., \& Mather, M. (2018). Locus Coeruleus Activity Strengthens Prioritized Memories Under Arousal. The Journal of Neuroscience, 38(6), 1558-1574. doi: 10.1523/jneurosci.2097-17.2017

Coltheart, M., Cox, R., Sowman, P., Morgan, H., Barnier, A., Langdon, R., ... Polito, V. (2018). Belief, delusion, hypnosis, and the right dorsolateral prefrontal cortex: A transcranial magnetic stimulation study. Cortex, 101, 234-248. doi: 10.1016/j.cortex.2018.01.001

Cristofori, I., Bulbulia, J., Shaver, J. H., Wilson, M., Krueger, F., \& Grafman, J. (2016). Neural correlates of mystical experience. Neuropsychologia, 80, 212-220. doi:

10.1016/j.neuropsychologia.2015.11.021

Dalton, M. A., Zeidman, P., Mccormick, C., \& Maguire, E. A. (2018). Differentiable Processing of Objects, Associations, and Scenes within the Hippocampus. The Journal of Neuroscience, 38(38), 8146-8159. doi: 10.1523/jneurosci.0263-18.2018

Darby, R. R., \& Pascual-Leone, A. (2017). Moral Enhancement Using Non-invasive Brain Stimulation. Frontiers in Human Neuroscience, 11. doi: 10.3389/fnhum.2017.00077

Denny, B. T., Inhoff, M. C., Zerubavel, N., Davachi, L., \& Ochsner, K. N. (2015). Getting Over It. Psychological Science, 26(9), 1377-1388. doi: 10.1177/0956797615578863

Dirac, P. A. M. (1940). XI.-The Relation between Mathematics and Physics. Proceedings of the Royal Society of Edinburgh, 59, 122-129. doi: 10.1017/s0370164600012207

Domenico, S. I. D., \& Ryan, R. M. (2017). The Emerging Neuroscience of Intrinsic Motivation: A New Frontier in Self-Determination Research. Frontiers in Human Neuroscience, 11. doi:

10.3389/fnhum.2017.00145

Dougherty, D. D., Chou, T., Buhlmann, U., Rauch, S. L., \& Deckersbach, T. (2017). Early Amygdala Activation and Later Ventromedial Prefrontal Cortex Activation During Anger Induction and Imagery. Journal of Medical Psychology, 1-8. doi: 10.3233/jmp-160002

Ekstrom, A. D., \& Ranganath, C. (2017). Space, time, and episodic memory: The hippocampus is all over the cognitive map. Hippocampus, 28(9), 680-687. doi: 10.1002/hipo.22750

Ezzati, A., Katz, M. J., Zammit, A. R., Lipton, M. L., Zimmerman, M. E., Sliwinski, M. J., \& Lipton, R. B. (2016). Differential association of left and right hippocampal volumes with verbal episodic and spatial memory in older adults. Neuropsychologia, 93, 380-385. doi:

10.1016/j.neuropsychologia.2016.08.016 
Fallon, S. J., Hampshire, A., Barker, R. A., \& Owen, A. M. (2016). Learning to be inflexible: Enhanced attentional biases in Parkinsons disease. Cortex, 82, 24-34. doi: 10.1016/j.cortex.2016.05.005

Fortune, D. \& K. (2009). Discover Your God-Given Gifts. Grand Rapids, MI: Chosen Books.

Floresco, S. B. (2015). The Nucleus Accumbens: An Interface Between Cognition, Emotion, and Action. Annual Review of Psychology, 66(1), 25-52. doi: 10.1146/annurev-psych-010213-115159

The Franklin Institute. (2019). Edison’s Lightbulb. Retrieved Nov. 5, 2019, from https://www.fi.edu/history-resources/edisons-lightbulb

Friedman, A., Homma, D., Gibb, L. G., Amemori, K.-I., Rubin, S. J., Hood, A. S., ... Graybiel, A. M. (2015). A Corticostriatal Path Targeting Striosomes Controls Decision-Making under Conflict. Cell, 161(6), 1320-1333. doi: 10.1016/j.cell.2015.04.049

Friedman, A., Homma, D., Bloem, B., Gibb, L. G., Amemori, K.-I., Hu, D., ... Graybiel, A. M. (2017). Chronic Stress Alters Striosome-Circuit Dynamics, Leading to Aberrant Decision-Making. Cell, 171(5). doi: 10.1016/j.cell.2017.10.017

Friesen, Lane (1986). Cognitive Styles in History: Contributor and Server. Victoria, BC: Lane Friesen, Inc.

Friesen, Lane (1986). Cognitive Styles in History: Perceiver and Mercy. Victoria, BC: Lane Friesen, Inc.

Friesen, Lorin (2012). God, Theology, and Cognitive Modules: A General Theory of Human Thought. Abbotsford, BC: Lorin Friesen

Friesen, Lorin (2015). Natural Cognitive Theology. Abbotsford, BC: Lorin Friesen.

Friesen, L., \& Van Dyke, A. (2013). A Cognitive Meta-Theory for TESOL. Retrieved from researchgate.net. doi: 10.13140/RG.2.1.3552.2721

Friesen, L., \& Van Dyke, A. (2015). A Cognitive Approach to Christianity and TESOL. Retrieved from researchgate.net. doi: 10.13140/RG.2.1.4338.7048

Furlan, M., Babcock, L., \& Vallesi, A. (2018). Decoding rule search domain in the left inferior frontal gyrus. Plos One, 13(3). doi: 10.1371/journal.pone.0194054

Ghaffari, H., Yoonessi, A., Darvishi, M. J., \& Ahmadi, A. (2018). Normal Electrical Activity of the Brain in Obsessive-Compulsive Patients After Anodal Stimulation of the Left Dorsolateral Prefrontal Cortex. Basic and Clinical Neuroscience Journal, 9(2), 135-146. doi: 10.29252/nirp.bcn.9.2.135

Gilam, G., Lin, T., Fruchter, E., \& Hendler, T. (2017). Neural indicators of interpersonal anger as cause and consequence of combat training stress symptoms. Psychological Medicine, 47(9), 1561-1572. doi: $10.1017 / \mathrm{s} 0033291716003354$

Gillan, C. M., Robbins, T. W., Sahakian, B. J., Heuvel, O. A. V. D., \& Wingen, G. V. (2016). The role of habit in compulsivity. European Neuropsychopharmacology, 26(5), 828-840. doi:

10.1016/j.euroneuro.2015.12.033 
Gómez-Jordana, L. I., Stafford, J., Peper, C. (L. E., \& Craig, C. M. (2018). Crossing Virtual Doors: A New Method to Study Gait Impairments and Freezing of Gait in Parkinson's Disease. Parkinsons Disease, 2018, 1-8. doi: 10.1155/2018/2957427

Gotink, R. A., Vernooij, M. W., Ikram, M. A., Niessen, W. J., Krestin, G. P., Hofman, A., ... Hunink, M. G. M. (2018). Meditation and yoga practice are associated with smaller right amygdala volume: the Rotterdam study. Brain Imaging and Behavior, 12(6), 1631-1639. doi: 10.1007/s11682-018-9826-z

Grabenhorst, F., \& Rolls, E. T. (2011). Value, pleasure and choice in the ventral prefrontal cortex. Trends in Cognitive Sciences, 15(2), 56-67. doi: 10.1016/j.tics.2010.12.004

Grajny, K., Pyata, H., Spiegel, K., Lacey, E. H., Xing, S., Brophy, C., \& Turkeltaub, P. E. (2016). Depression Symptoms in Chronic Left Hemisphere Stroke Are Related to Dorsolateral Prefrontal Cortex Damage. The Journal of Neuropsychiatry and Clinical Neurosciences, 28(4), 292-298. doi: 10.1176/appi.neuropsych.16010004

Graybiel, A., \& Smith, K. (2014). GOOD HABITS, BAD HABITS. Scientific American, 310(6), 38-43. Retrieved from https://www.jstor.org/stable/26039932

Graybiel, A. M., \& Grafton, S. T. (2015). The Striatum: Where Skills and Habits Meet. Cold Spring Harbor Perspectives in Biology, 7(8). doi: 10.1101/cshperspect.a021691

Green, A. E., Spiegel, K. A., Giangrande, E. J., Weinberger, A. B., Gallagher, N. M., \& Turkeltaub, P. E. (2016). Thinking Cap Plus Thinking Zap: tDCS of Frontopolar Cortex Improves Creative Analogical Reasoning and Facilitates Conscious Augmentation of State Creativity in Verb Generation. Cerebral Cortex. doi: 10.1093/cercor/bhw080

Grover, T., Georgiev, D., Kalliola, R., Mahlknecht, P., Zacharia, A., Candelario, J., ... Tripoliti, E. (2019). Effect of Low versus High Frequency Subthalamic Deep Brain Stimulation on Speech Intelligibility and Verbal Fluency in Parkinson's Disease: A Double-Blind Study. Journal of Parkinsons Disease, 9(1), 141-151. doi: 10.3233/jpd-181368

Guex, R., Hofstetter, C., Domínguez-Borràs, J., Méndez-Bértolo, C., Sterpenich, V., Spinelli, L., ... Vuilleumier, P. (2019). Neurophysiological evidence for early modulation of amygdala activity by emotional reappraisal. Biological Psychology, 145, 211-223. doi: 10.1016/j.biopsycho.2019.05.006

Hadjikhani, N., Johnels, J. Å., Zürcher, N. R., Lassalle, A., Guillon, Q., Hippolyte, L., ... Gillberg, C. (2017). Look me in the eyes: constraining gaze in the eye-region provokes abnormally high subcortical activation in autism. Scientific Reports, 7(1). doi: 10.1038/s41598-017-03378-5

Hardee, J. E., Thompson, J. C., \& Puce, A. (2008). The left amygdala knows fear: laterality in the amygdala response to fearful eyes. Social Cognitive and Affective Neuroscience, 3(1), 47-54. doi: 10.1093/scan/nsn001

Hartogsveld, B., Bramson, B., Vijayakumar, S., Campen, A. D. V., Marques, J. P., Roelofs, K., ... Mars, R. B. (2018). Lateral frontal pole and relational processing: Activation patterns and connectivity profile. Behavioural Brain Research, 355, 2-11. doi: 10.1016/j.bbr.2017.08.003 
Hartwright, C. E., Apperly, I. A., \& Hansen, P. C. (2014). Representation, Control, or Reasoning? Distinct Functions for Theory of Mind within the Medial Prefrontal Cortex. Journal of Cognitive Neuroscience, 26(4), 683-698. doi: 10.1162/jocn_a_00520

Harty, S., Robertson, I. H., Miniussi, C., Sheehy, O. C., Devine, C. A., Mccreery, S., \& Oconnell, R. G. (2014). Transcranial Direct Current Stimulation over Right Dorsolateral Prefrontal Cortex Enhances Error Awareness in Older Age. Journal of Neuroscience, 34(10), 3646-3652. doi: 10.1523/jneurosci.5308-13.2014

He, M., Qi, C., Lu, Y., Song, A., Hayat, S. Z., \& Xu, X. (2018). The Sport Expert’s Attention Superiority on Skill-related Scene Dynamic by the Activation of left Medial Frontal Gyrus: An ERP and LORETA Study. Neuroscience, 379, 93-102. doi: 10.1016/j.neuroscience.2018.02.043

Holz, N. E., Boecker, R., Jennen-Steinmetz, C., Buchmann, A. F., Blomeyer, D., Baumeister, S., ... Laucht, M. (2016). Positive coping styles and perigenual ACC volume: two related mechanisms for conferring resilience? Social Cognitive and Affective Neuroscience, 11(5), 813-820. doi: 10.1093/scan/ nsw005

Hori, G.Victor Sōgen. 2000. Kōan and kenshō in the Rinzai Zen curriculum. In The kōan: Texts and contexts in Zen Buddhism, ed. Steven Heine and Dale S. Wright. New York: Oxford University Press, pp. 280-315.

Howell, L. L., \& Cunningham, K. A. (2014). Serotonin 5-HT2 Receptor Interactions with Dopamine Function: Implications for Therapeutics in Cocaine Use Disorder. Pharmacological Reviews, 67(1), 176-197. doi: 10.1124/pr.114.009514

Hussey, E. K., Ward, N., Christianson, K., \& Kramer, A. F. (2015). Language and Memory Improvements following tDCS of Left Lateral Prefrontal Cortex. Plos One, 10(11). doi: 10.1371/journal.pone.0141417

Hutchinson, L. (2019, September 23). Video: Ars talks Civilization with the man himself: Sid Meier. Retrieved from https://arstechnica.com/gaming/2019/09/video-ars-talks-civilization-with-the-manhimself-sid-meier/.

Ishii, M., \& Okuyama, K. (2017). Characteristics associated with freezing of gait in actual daily living in Parkinson's disease. Journal of Physical Therapy Science, 29(12), 2151-2156. doi:

10.1589/jpts.29.2151

Jenni, N. L., Larkin, J. D., \& Floresco, S. B. (2017). Prefrontal Dopamine D1 and D2 Receptors Regulate Dissociable Aspects of Decision Making via Distinct Ventral Striatal and Amygdalar Circuits. The Journal of Neuroscience, 37(26), 6200-6213. doi: 10.1523/jneurosci.0030-17.2017

Jin, X., \& Costa, R. M. (2015). Shaping action sequences in basal ganglia circuits. Current Opinion in Neurobiology, 33, 188-196. doi: 10.1016/j.conb.2015.06.011

Kellmeyer, P., Vry, M.-S., \& Ball, T. (2016). A transcallosal fiber system between homotopic inferior frontal regions supports complex linguistic processing. doi: 10.1101/079244 
Kim, M., \& Maguire, E. A. (2018). Hippocampus, Retrosplenial and Parahippocampal Cortices Encode Multicompartment 3D Space in a Hierarchical Manner. Cerebral Cortex, 28(5), 1898-1909. doi: 10.1093/cercor/bhy054

Kirk, U., Skov, M., Christensen, M. S., \& Nygaard, N. (2009). Brain correlates of aesthetic expertise: A parametric fMRI study. Brain and Cognition, 69(2), 306-315. doi: 10.1016/j.bandc.2008.08.004

Knoch, D., Gianotti, L. R. R., Pascual-Leone, A., Treyer, V., Regard, M., Hohmann, M., \& Brugger, P. (2006). Disruption of Right Prefrontal Cortex by Low-Frequency Repetitive Transcranial Magnetic Stimulation Induces Risk-Taking Behavior. Journal of Neuroscience, 26(24), 6469-6472. doi: 10.1523/ jneurosci.0804-06.2006

Knoch, D., Pascual-Leone, A., Meyer, K., Treyer, V., \& Fehr, E. (2006). Diminishing Reciprocal Fairness by Disrupting the Right Prefrontal Cortex. Science, 314(5800), 829-832. doi: 10.1126/science.1129156

Kolling, N., Behrens, T., Wittmann, M., \& Rushworth, M. (2016). Multiple signals in anterior cingulate cortex. Current Opinion in Neurobiology, 37, 36-43. doi: 10.1016/j.conb.2015.12.007

Kolling, N., Scholl, J., Chekroud, A., Trier, H. A., \& Rushworth, M. F. (2018). Prospection, Perseverance, and Insight in Sequential Behavior. Neuron, 99(5). doi: 10.1016/j.neuron.2018.08.018 Lagravinese, G., Pelosin, E., Bonassi, G., Carbone, F., Abbruzzese, G., \& Avanzino, L. (2018). Gait initiation is influenced by emotion processing in Parkinsons disease patients with freezing. Movement Disorders, 33(4), 609-617. doi: 10.1002/mds.27312

Lawrence M. Krauss Quotes. (n.d.). BrainyQuote.com. Retrieved November 6, 2019, from BrainyQuote.com Web site: https://www.brainyquote.com/quotes/lawrence_m_krauss_526731

Kringelbach, M. L., \& Berridge, K. C. (2017). The Affective Core of Emotion: Linking Pleasure, Subjective Well-Being, and Optimal Metastability in the Brain. Emotion Review, 9(3), 191-199. doi: 10.1177/1754073916684558

Kuhn, T. S. (1970). The Structure of Scientific Revolutions (2nd Ed.). Chicago: University of Chicago Press

Lipski, W. J., Alhourani, A., Pirnia, T., Jones, P. W., Dastolfo-Hromack, C., Helou, L. B., ... Richardson, R. M. (2018). Subthalamic Nucleus Neurons Differentially Encode Early and Late Aspects of Speech Production. The Journal of Neuroscience, 38(24), 5620-5631. doi: 10.1523/jneurosci.348017.2018

Liu, R. T., Kleiman, E. M., Nestor, B. A., \& Cheek, S. M. (2015). The Hopelessness Theory of Depression: A Quarter-Century in Review. Clinical Psychology: Science and Practice, 22(4), 345-365. doi: $10.1111 /$ cpsp.12125

Lombardi, W. J., Andreason, P. J., Sirocco, K. Y., Rio, D. E., Gross, R. E., Umhau, J. C., \& Hommer, D. W. (1999). Wisconsin Card Sorting Test Performance Following Head Injury: Dorsolateral FrontoStriatal Circuit Activity Predicts Perseveration. Journal of Clinical and Experimental Neuropsychology, 21(1), 2-16. doi: 10.1076/jcen.21.1.2.940 
López-Giménez, J. F., Mengod, G., Palacios, J. M., \& Vilaró, M. T. (1999). Human striosomes are enriched in 5-HT2Areceptors: autoradiographical visualization with [3H]MDL100,907, [125I]( \pm )DOI and [3H]ketanserin. European Journal of Neuroscience, 11(10), 3761-3765. doi: 10.1046/j.14609568.1999.00827.x

López-Giménez, J. F., \& González-Maeso, J. (2017). Hallucinogens and Serotonin 5-HT2A ReceptorMediated Signaling Pathways. Behavioral Neurobiology of Psychedelic Drugs Current Topics in Behavioral Neurosciences, 45-73. doi: 10.1007/7854_2017_478

Mack, M. L., Love, B. C., \& Preston, A. R. (2018). Building concepts one episode at a time: The hippocampus and concept formation. Neuroscience Letters, 680, 31-38. doi:

10.1016/j.neulet.2017.07.061

Maguire, E. A., Spiers, H. J., Good, C. D., Hartley, T., Frackowiak, R. S., \& Burgess, N. (2003). Navigation expertise and the human hippocampus: A structural brain imaging analysis. Hippocampus, 13(2), 250-259. doi: 10.1002/hipo.10087

Maguire, E. A., Intraub, H., \& Mullally, S. L. (2016). Scenes, Spaces, and Memory Traces. The Neuroscientist, 22(5), 432-439. doi: 10.1177/1073858415600389

Manzano, Ö. D., Cervenka, S., Jucaite, A., Hellenäs, O., Farde, L., \& Ullén, F. (2013). Individual differences in the proneness to have flow experiences are linked to dopamine D2-receptor availability in the dorsal striatum. NeuroImage, 67, 1-6. doi: 10.1016/j.neuroimage.2012.10.072

Maréchal, M. A., Cohn, A., Ugazio, G., \& Ruff, C. C. (2017). Increasing honesty in humans with noninvasive brain stimulation. Proceedings of the National Academy of Sciences, 114(17), 4360-4364. doi: 10.1073/pnas.1614912114

Melloni, L., Leeuwen, S. V., Alink, A., \& Müller, N. G. (2012). Interaction between Bottom-up Saliency and Top-down Control: How Saliency Maps Are Created in the Human Brain. Cerebral Cortex, 22(12), 2943-2952. doi: 10.1093/cercor/bhr384

Meyer, M. L., \& Lieberman, M. D. (2018). Why People Are Always Thinking about Themselves: Medial Prefrontal Cortex Activity during Rest Primes Self-referential Processing. Journal of Cognitive Neuroscience, 30(5), 714-721. doi: 10.1162/jocn_a_01232

Milivojevic, B., Vicente-Grabovetsky, A., \& Doeller, C. F. (2015). Insight Reconfigures HippocampalPrefrontal Memories. Current Biology, 25(7), 821-830. doi: 10.1016/j.cub.2015.01.033

Mitchell, A. S. (2015). The mediodorsal thalamus as a higher order thalamic relay nucleus important for learning and decision-making. Neuroscience \& Biobehavioral Reviews, 54, 76-88. doi: 10.1016/j.neubiorev.2015.03.001

Mitchell, J. P., Banaji, M. R., \& Macrae, C. N. (2005). The Link between Social Cognition and Selfreferential Thought in the Medial Prefrontal Cortex. Journal of Cognitive Neuroscience, 17(8), 13061315. doi: 10.1162/0898929055002418 
Morawetz, C., Bode, S., Baudewig, J., Kirilina, E., \& Heekeren, H. R. (2015). Changes in Effective Connectivity Between Dorsal and Ventral Prefrontal Regions Moderate Emotion Regulation. Cerebral Cortex, 26(5), 1923-1937. doi: 10.1093/cercor/bhv005

Morawetz, C., Bode, S., Baudewig, J., \& Heekeren, H. R. (2016). Effective amygdala-prefrontal connectivity predicts individual differences in successful emotion regulation. Social Cognitive and Affective Neuroscience, 12(4), 569-585. doi: 10.1093/scan/nsw169

Motzkin, J. C., Philippi, C. L., Wolf, R. C., Baskaya, M. K., \& Koenigs, M. (2015). Ventromedial Prefrontal Cortex Is Critical for the Regulation of Amygdala Activity in Humans. Biological Psychiatry, 77(3), 276-284. doi: 10.1016/j.biopsych.2014.02.014

Murphy, E. R., Barch, D. M., Pagliaccio, D., Luby, J. L., \& Belden, A. C. (2016). Functional connectivity of the amygdala and subgenual cingulate during cognitive reappraisal of emotions in children with MDD history is associated with rumination. Developmental Cognitive Neuroscience, 18, 89-100. doi: 10.1016/j.dcn.2015.11.003

Murray, L. J., \& Ranganath, C. (2007). The Dorsolateral Prefrontal Cortex Contributes to Successful Relational Memory Encoding. Journal of Neuroscience, 27(20), 5515-5522. doi:

10.1523/jneurosci.0406-07.2007

Nakajima, M., \& Halassa, M. M. (2017). Thalamic control of functional cortical connectivity. Current Opinion in Neurobiology, 44, 127-131. doi: 10.1016/j.conb.2017.04.001

Nakamura, T., Matsui, T., Utsumi, A., Yamazaki, M., Makita, K., Harada, T., ... Sadato, N. (2017). The role of the amygdala in incongruity resolution: the case of humor comprehension. Social Neuroscience, 13(5), 553-565. doi: 10.1080/17470919.2017.1365760

Nejad, A. B., Rotgé, J.-Y., Valabregue, R., Guérin-Langlois, C., Hoertel, N., Gorwood, P., ... Lemogne, C. (2019). Medial prefrontal disengagement during self-focus in formerly depressed patients prone to rumination. Journal of Affective Disorders, 247, 36-44. doi: 10.1016/j.jad.2019.01.004

Nielson, D. M., Smith, T. A., Sreekumar, V., Dennis, S., \& Sederberg, P. B. (2015). Human hippocampus represents space and time during retrieval of real-world memories. Proceedings of the National Academy of Sciences, 112(35), 11078-11083. doi: 10.1073/pnas.1507104112

Nour, M. M., Dahoun, T., Schwartenbeck, P., Adams, R. A., Fitzgerald, T. H. B., Coello, C., ... Howes, O. D. (2018). Dopaminergic basis for signaling belief updates, but not surprise, and the link to paranoia. Proceedings of the National Academy of Sciences, 115(43). doi: 10.1073/pnas.1809298115

Nyhus, E., \& Badre, D. (2015). Memory Retrieval and the Functional Organization of Frontal Cortex. The Wiley Handbook on the Cognitive Neuroscience of Memory, 131-149. doi: 10.1002/9781118332634.ch7

Obeso, I., Wilkinson, L., Casabona, E., Speekenbrink, M., Bringas, M. L., Álvarez, M., ... Jahanshahi, M. (2014). The subthalamic nucleus and inhibitory control: impact of subthalamotomy in Parkinson's disease. Brain, 137(5), 1470-1480. doi: 10.1093/brain/awu058 
Ochsner, K. N., Ray, R. R., Hughes, B., Mcrae, K., Cooper, J. C., Weber, J., ... Gross, J. J. (2009). Bottom-Up and Top-Down Processes in Emotion Generation. Psychological Science, 20(11), 13221331. doi: 10.1111/j.1467-9280.2009.02459.x

Olson, I. R., Heide, R. J. V. D., Alm, K. H., \& Vyas, G. (2015). Development of the uncinate fasciculus: Implications for theory and developmental disorders. Developmental Cognitive Neuroscience, 14, 5061. doi: 10.1016/j.dcn.2015.06.003

Papousek, I., Weiss, E. M., Perchtold, C. M., Weber, H., Assunção, V. L. D., Schulter, G., ... Fink, A. (2016). The capacity for generating cognitive reappraisals is reflected in asymmetric activation of frontal brain regions. Brain Imaging and Behavior, 11(2), 577-590. doi: 10.1007/s11682-016-9537-2

Park, S.-E., Choi, N.-G., \& Jeong, G.-W. (2016). Metabolic abnormality in the right dorsolateral prefrontal cortex in patients with obsessive-compulsive disorder: proton magnetic resonance spectroscopy. Acta Neuropsychiatrica, 29(3), 164-169. doi: 10.1017/neu.2016.48

Passecker, J., Mikus, N., Malagon-Vina, H., Anner, P., Dimidschstein, J., Fishell, G., ... Klausberger, T. (2019). Activity of Prefrontal Neurons Predict Future Choices during Gambling. Neuron, 101(1). doi: 10.1016/j.neuron.2018.10.050

Patil, I., Zucchelli, M. M., Kool, W., Campbell, S., Ffornasier, F., Calo, M., ... Cushman, F. A. (2018). Reasoning supports utilitarian resolutions to moral dilemmas across diverse measures. doi: 10.31234/osf.io/q86vx

Poppenk, J., Evensmoen, H. R., Moscovitch, M., \& Nadel, L. (2013). Long-axis specialization of the human hippocampus. Trends in Cognitive Sciences, 17(5), 230-240. doi: 10.1016/j.tics.2013.03.005

Robertson, C. L., Ishibashi, K., Mandelkern, M. A., Brown, A. K., Ghahremani, D. G., Sabb, F., ... London, E. D. (2015). Striatal D1- and D2-type Dopamine Receptors Are Linked to Motor Response Inhibition in Human Subjects. The Journal of Neuroscience, 35(15), 5990-5997. doi: 10.1523/jneurosci.4850-14.2015

Rodenkirch, C., Liu, Y., Schriver, B. J., \& Wang, Q. (2018). Locus coeruleus activation enhances thalamic feature selectivity via norepinephrine regulation of intrathalamic circuit dynamics. Nature Neuroscience, 22(1), 120-133. doi: 10.1038/s41593-018-0283-1

Rolls, E. T. (2015). Limbic systems for emotion and for memory, but no single limbic system. Cortex, 62, 119-157. doi: 10.1016/j.cortex.2013.12.005

Rolls, E. T. (2017a). Evolution of the Emotional Brain. Evolution of the Brain, Cognition, and Emotion in Vertebrates, 251-272. doi: 10.1007/978-4-431-56559-8_12

Rolls, E. T. (2017b). Neurobiological foundations of aesthetics and art. New Ideas in Psychology, 47, 121-135. doi: 10.1016/j.newideapsych.2017.03.005

Rolls, E. T. (2019a). The cingulate cortex and limbic systems for emotion, action, and memory. Brain Structure and Function. doi: 10.1007/s00429-019-01945-2

Rolls, E. T. (2019b). The orbitofrontal cortex and emotion in health and disease, including depression. Neuropsychologia, 128, 14-43. doi: 10.1016/j.neuropsychologia.2017.09.021 
Rosa, P. A. D., Catricalà, E., Canini, M., Vigliocco, G., \& Cappa, S. F. (2018). The left inferior frontal gyrus: A neural crossroads between abstract and concrete knowledge. NeuroImage, 175, 449-459. doi: 10.1016/j.neuroimage.2018.04.021

Rose, S. (2019, April). Do Microdoses of LSD Change Your Mind? Scientific American. Retrieved from https://www.scientificamerican.com/article/do-microdoses-of-lsd-change-your-mind/

Rueckemann, J. W., \& Buffalo, E. A. (2017). Spatial responses, immediate experience, and memory in the monkey hippocampus. Current Opinion in Behavioral Sciences, 17, 155-160. doi:

10.1016/j.cobeha.2017.08.008

Rutishauser, U., Tudusciuc, O., Neumann, D., Mamelak, A. N., Heller, A. C., Ross, I. B., ... Adolphs, R. (2011). Single-Unit Responses Selective for Whole Faces in the Human Amygdala. Current Biology, 21(19), 1654-1660. doi: 10.1016/j.cub.2011.08.035

Saalmann, Y. B., \& Kastner, S. (2011). Cognitive and Perceptual Functions of the Visual Thalamus. Neuron, 71(2), 209-223. doi: 10.1016/j.neuron.2011.06.027

Sadato, N. (2017). Shared Attention and Interindividual Neural Synchronization in the Human Right Inferior Frontal Cortex. The Prefrontal Cortex as an Executive, Emotional, and Social Brain, 207-225. doi: 10.1007/978-4-431-56508-6_11

Schein, C., \& Gray, K. (2015). The eyes are the window to the uncanny valley: Mind perception, autism and missing souls. Interaction Studies Interaction Studies Social Behaviour and Communication in Biological and Artificial Systems, 16(2), 173-179. doi: 10.1075/is.16.2.02sch

Schoenbaum, G., Stalnaker, T. A., \& Niv, Y. (2013). How Did the Chicken Cross the Road? With Her Striatal Cholinergic Interneurons, Of Course. Neuron, 79(1), 3-6. doi: 10.1016/j.neuron.2013.06.033

Scholl, J., \& Rushworth, M. F. (2017). Obsessing about Uncertainty? Neuron, 96(2), 250-252. doi: 10.1016/j.neuron.2017.09.045

Schuck, N. W., Wilson, R., \& Niv, Y. (2017). A State Representation for Reinforcement Learning and Decision-Making in the Orbitofrontal Cortex. Doi: 10.1101/210591

Schurz, M., Radua, J., Aichhorn, M., Richlan, F., \& Perner, J. (2014). Fractionating theory of mind: A meta-analysis of functional brain imaging studies. Neuroscience \& Biobehavioral Reviews, 42, 9-34. doi: 10.1016/j.neubiorev.2014.01.009

Sebastian, A., Jung, P., Neuhoff, J., Wibral, M., Fox, P. T., Lieb, K., ... Mobascher, A. (2015).

Dissociable attentional and inhibitory networks of dorsal and ventral areas of the right inferior frontal cortex: a combined task-specific and coordinate-based meta-analytic fMRI study. Brain Structure and Function, 221(3), 1635-1651. doi: 10.1007/s00429-015-0994-y

Sekeres, M. J., Winocur, G., \& Moscovitch, M. (2018). The hippocampus and related neocortical structures in memory transformation. Neuroscience Letters, 680, 39-53. doi:

10.1016/j.neulet.2018.05.006 
Sergerie, K., Chochol, C., \& Armony, J. L. (2008). The role of the amygdala in emotional processing: A quantitative meta-analysis of functional neuroimaging studies. Neuroscience \& Biobehavioral Reviews, 32(4), 811-830. doi: 10.1016/j.neubiorev.2007.12.002

Sheldon, S., Mcandrews, M. P., Pruessner, J., \& Moscovitch, M. (2016). Dissociating patterns of anterior and posterior hippocampal activity and connectivity during distinct forms of category fluency. Neuropsychologia, 90, 148-158. doi: 10.1016/j.neuropsychologia.2016.06.028

Simanova, I., Francken, J. C., Lange, F. P. D., \& Bekkering, H. (2015). Linguistic priors shape categorical perception. Language, Cognition and Neuroscience, 31(1), 159-165. doi: 10.1080/23273798.2015.1072638

Smith, R., Alkozei, A., Bao, J., \& Killgore, W. D. S. (2017). Successful Goal-Directed Memory Suppression is Associated With Increased Inter-Hemispheric Coordination Between Right and Left Frontoparietal Control Networks. Psychological Reports, 121(1), 93-111. doi: 10.1177/0033294117723018

Spain, D., Sin, J., Harwood, L., Mendez, M. A., \& Happé, F. (2017). Cognitive behaviour therapy for social anxiety in autism spectrum disorder: a systematic review. Advances in Autism, 3(1), 34-46. doi: 10.1108/aia-07-2016-0020

Starkstein, S., Dragovic, M., Brockman, S., Wilson, M., Bruno, V., \& Merello, M. (2015). The Impact of Emotional Distress on Motor Blocks and Festination in Parkinson's Disease. The Journal of Neuropsychiatry and Clinical Neurosciences, 27(2), 121-126. doi: 10.1176/appi.neuropsych.13030053 Stewart-Williams, S., \& Halsey, L. G. (2018). Men, Women, and Science: Why the Differences and What Should Be Done? doi: 10.31234/osf.io/ms524

Sturm, V. E., Sollberger, M., Seeley, W. W., Rankin, K. P., Ascher, E. A., Rosen, H. J., ... Levenson, R. W. (2012). Role of right pregenual anterior cingulate cortex in self-conscious emotional reactivity. Social Cognitive and Affective Neuroscience, 8(4), 468-474. doi: 10.1093/scan/nss023

Supekar, K., Iuculano, T., Chen, L., \& Menon, V. (2015). Remediation of Childhood Math Anxiety and Associated Neural Circuits through Cognitive Tutoring. Journal of Neuroscience, 35(36), 1257412583. doi: 10.1523/jneurosci.0786-15.2015

Takamura, T., Nishitani, S., \& Shinohara, K. (2016). Possible neural correlate of young child attachment to mother in 4 to 5 year olds. Acta Med. Nagasaki, 60, 45-51. doi: 10.11343/amn.60.45

Tecuapetla, F., Jin, X., Lima, S. Q., \& Costa, R. M. (2016). Complementary Contributions of Striatal Projection Pathways to Action Initiation and Execution. Cell, 166(3), 703-715. doi:

10.1016/j.cell.2016.06.032

Tervo, D. G., Proskurin, M., Manakov, M., Kabra, M., Vollmer, A., Branson, K., \& Karpova, A. Y. (2014). Behavioral Variability through Stochastic Choice and Its Gating by Anterior Cingulate Cortex. Cell, 159(1), 21-32. doi: 10.1016/j.cell.2014.08.037 
Thieba, C., Long, X., Dewey, D., \& Lebel, C. (2019). Young children in different linguistic environments: A multimodal neuroimaging study of the inferior frontal gyrus. Brain and Cognition, 134, 71-79. doi: 10.1016/j.bandc.2018.05.009

Tompary, A., \& Davachi, L. (2017). Consolidation Promotes the Emergence of Representational Overlap in the Hippocampus and Medial Prefrontal Cortex. Neuron, 96(1). doi:

10.1016/j.neuron.2017.09.005

Tottenham, N., \& Gabard-Durnam, L. J. (2017a). The developing amygdala: a student of the world and a teacher of the cortex. Current Opinion in Psychology, 17, 55-60. doi: 10.1016/j.copsyc.2017.06.012

Tottenham, N. (2017b). The Fundamental Role of Early Environments to Developing an Emotionally Healthy Brain. Policy Insights from the Behavioral and Brain Sciences, 5(1), 98-103. doi:

$10.1177 / 2372732217745098$

Tulviste, J., \& Bachmann, T. (2018). Diminished Risk-Aversion After Right DLPFC Stimulation: Effects of rTMS on a Risky Ball Throwing Task. Journal of the International Neuropsychological Society, 25(1), 72-78. doi: 10.1017/s1355617718000930

Turnbull, A., Wang, H. T., Murphy, C., Ho, N. S. P., Wang, X., Sormaz, M., ... Smallwood, J. (2019). Left dorsolateral prefrontal cortex supports context-dependent prioritisation of off-task thought. Nature Communications, 10(1). doi: 10.1038/s41467-019-11764-y

Ueda, Y., Yamanaka, K., Noritake, A., Enomoto, K., Matsumoto, N., Yamada, H., ... Kimura, M. (2017). Distinct Functions of the Primate Putamen Direct and Indirect Pathways in Adaptive OutcomeBased Action Selection. Frontiers in Neuroanatomy, 11. doi: 10.3389/fnana.2017.00066

Uncapher, M. R., Hutchinson, J. B., \& Wagner, A. D. (2011). Dissociable Effects of Top-Down and Bottom-Up Attention during Episodic Encoding. Journal of Neuroscience, 31(35), 12613-12628. doi: 10.1523/jneurosci.0152-11.2011

Urbanski, M., Bréchemier, M.-L., Garcin, B., Bendetowicz, D., Schotten, M. T. D., Foulon, C., ... Volle, E. (2016). Reasoning by analogy requires the left frontal pole: lesion-deficit mapping and clinical implications. Brain, 139(6), 1783-1799. doi: 10.1093/brain/aww072

Vaessen, T. (2018). Stress Sensitivity in Psychosis: Assessment, Mechanism \& Intervention. Enschede: Gildeprint Drukkerijen. https://doi.org/10.26481/dis.20180613tv

Vaghi, M. M., Luyckx, F., Sule, A., Fineberg, N. A., Robbins, T. W., \& Martino, B. D. (2017). Compulsivity Reveals a Novel Dissociation between Action and Confidence. Neuron, 96(2). doi: 10.1016/j.neuron.2017.09.006

Varazzani, C., San-Galli, A., Gilardeau, S., \& Bouret, S. (2015). Noradrenaline and Dopamine Neurons in the Reward/Effort Trade-Off: A Direct Electrophysiological Comparison in Behaving Monkeys. Journal of Neuroscience, 35(20), 7866-7877. doi: 10.1523/jneurosci.0454-15.2015

Verharen, J. P. H., Adan, R. A. H., \& Vanderschuren, L. J. M. J. (2019). Differential contributions of striatal dopamine D1 and D2 receptors to component processes of value-based decision making. Neuropsychopharmacology. doi: 10.1038/s41386-019-0454-0 
Volkow, N. D., \& Baler, R. D. (2015). NOW vs LATER brain circuits: implications for obesity and addiction. Trends in Neurosciences, 38(6), 345-352. doi: 10.1016/j.tins.2015.04.002

Wagner, D. D., Haxby, J. V., \& Heatherton, T. F. (2012). The representation of self and person knowledge in the medial prefrontal cortex. Wiley Interdisciplinary Reviews: Cognitive Science, 3(4), 451-470. doi: 10.1002/wcs.1183

Wang, L., Amalric, M., Fang, W., Jiang, X., Pallier, C., Figueira, S., ... Dehaene, S. (2019).

Representation of spatial sequences using nested rules in human prefrontal cortex. NeuroImage, 186, 245-255. doi: 10.1016/j.neuroimage.2018.10.061

Weymar, M., \& Schwabe, L. (2016). Amygdala and Emotion: The Bright Side of It. Frontiers in Neuroscience, 10. doi: 10.3389/fnins.2016.00224

Wirz, L., Bogdanov, M., \& Schwabe, L. (2018). Habits under stress: mechanistic insights across different types of learning. Current Opinion in Behavioral Sciences, 20, 9-16. doi:

10.1016/j.cobeha.2017.08.009

Wu, X., Xu, F., Chen, X., Wang, L., Huang, W., Wan, K., ... Wang, K. (2018). The Effect of HighDefinition Transcranial Direct Current Stimulation of the Right Inferior Frontal Gyrus on Empathy in Healthy Individuals. Frontiers in Human Neuroscience, 12. doi: 10.3389/fnhum.2018.00446

Wunderlich, K., Dayan, P., \& Dolan, R. J. (2012). Mapping value based planning and extensively trained choice in the human brain. Nature Neuroscience, 15(5), 786-791. doi: 10.1038/nn.3068

Xu, H., Wang, P., Ye, Z. E., Di, X., Xu, G., Mo, L., ... Jin, H. (2016). The Role of Medial Frontal Cortex in Action Anticipation in Professional Badminton Players. Frontiers in Psychology, 7. doi: 10.3389/fpsyg.2016.01817

Yamamoto, D. J., Woo, C.-W., Wager, T. D., Regner, M. F., \& Tanabe, J. (2015). Influence of dorsolateral prefrontal cortex and ventral striatum on risk avoidance in addiction: A mediation analysis. Drug and Alcohol Dependence, 149, 10-17. doi: 10.1016/j.drugalcdep.2014.12.026

Yamanaka, K., Hori, Y., Minamimoto, T., Yamada, H., Matsumoto, N., Enomoto, K., ... Kimura, M. (2017). Roles of centromedian parafascicular nuclei of thalamus and cholinergic interneurons in the dorsal striatum in associative learning of environmental events. Journal of Neural Transmission, 125(3), 501-513. doi: 10.1007/s00702-017-1713-z

Yin, H. H., \& Knowlton, B. J. (2006). The role of the basal ganglia in habit formation. Nature Reviews Neuroscience, 7(6), 464-476. doi: 10.1038/nrn1919

Yokoyama, O., Miura, N., Watanabe, J., Takemoto, A., Uchida, S., Sugiura, M., ... Nakamura, K. (2010). Right frontopolar cortex activity correlates with reliability of retrospective rating of confidence in short-term recognition memory performance. Neuroscience Research, 68(3), 199-206. doi: 10.1016/j.neures.2010.07.2041

Zajkowski, W. K., Kossut, M., \& Wilson, R. C. (2017). A causal role for right frontopolar cortex in directed, but not random, exploration. ELife, 6. doi: 10.7554/elife.27430 
Zavorotnyy, M., Zöllner, R., Schulte-Güstenberg, L. R., Wulff, L., Schöning, S., Dannlowski, U., ... Konrad, C. (2017). Low left amygdala volume is associated with a longer duration of unipolar depression. Journal of Neural Transmission, 125(2), 229-238. doi: 10.1007/s00702-017-1811-y

Zeidman, P., Mullally, S. L., \& Maguire, E. A. (2014). Constructing, Perceiving, and Maintaining Scenes: Hippocampal Activity and Connectivity. Cerebral Cortex, 25(10), 3836-3855. doi:

10.1093/cercor/bhu266

Zeidman, P., \& Maguire, E. A. (2016). Anterior hippocampus: the anatomy of perception, imagination and episodic memory. Nature Reviews Neuroscience, 17(3), 173-182. doi: 10.1038/nrn.2015.24

Zeki, S., Romaya, J. P., Benincasa, D. M. T., \& Atiyah, M. F. (2014). The experience of mathematical beauty and its neural correlates. Frontiers in Human Neuroscience, 8. doi: 10.3389/fnhum.2014.00068

Zeki, S., Chén, O. Y., \& Romaya, J. P. (2018). The Biological Basis of Mathematical Beauty. Frontiers in Human Neuroscience, 12. doi: 10.3389/fnhum.2018.00467

Zeki, S. (2019). Notes Towards a (Neurobiological) Definition of Beauty. Gestalt Theory, 41(2), 107112. doi: 10.2478/gth-2019-0012

Zhang, W., He, X., Lai, S., Wan, J., Lai, S., Zhao, X., \& Li, D. (2017). Neural substrates of embodied natural beauty and social endowed beauty: An fMRI study. Scientific Reports, 7(1). doi:

10.1038/s41598-017-07608-8

Zhu, F. F., Yeung, A. Y., Poolton, J. M., Lee, T. M., Leung, G. K., \& Masters, R. S. (2015). Cathodal Transcranial Direct Current Stimulation Over Left Dorsolateral Prefrontal Cortex Area Promotes Implicit Motor Learning in a Golf Putting Task. Brain Stimulation, 8(4), 784-786. doi:

10.1016/j.brs.2015.02.005

Zikopoulos, B., \& Barbas, H. (2012). Pathways for Emotions and Attention Converge on the Thalamic Reticular Nucleus in Primates. Journal of Neuroscience, 32(15), 5338-5350. doi:

10.1523/jneurosci.4793-11.2012 